\author{
Aus der Abteilung Neuro- und Sinnesphysiologie \\ (Prof. Dr. med. D. W. Richter) \\ im Zentrum Physiologie und Pathophysiologie \\ der Medizinischen Fakultät der Universität Göttingen
}

\title{
Optical Analysis of Mitochondrial Function and Heterogeneity in Cultured Hippocampal Astrocytes
}

\author{
INAUGURAL-DISSERTATION \\ zur Erlangung des Doktorgrades \\ der Medizinischen Fakultät der \\ Georg-August-Universität zu Göttingen
}

vorgelegt von

Vera Catharina Wilma Keil

aus

Münster/Deutschland

Göttingen 2009 
Dekan:

I. Berichterstatter:

II. Berichterstatter/in:

III. Berichterstatter/in:

Tag der mündlichen Prüfung:
Prof. Dr. med. C. Frömmel

JProf. Dr. rer. nat. M. Müller 


\section{Contents}

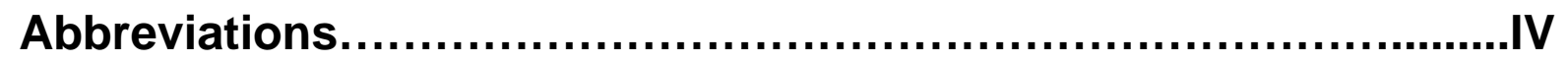

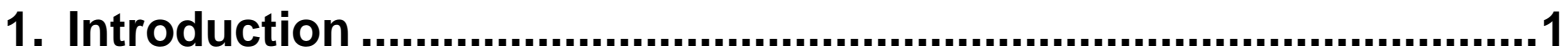

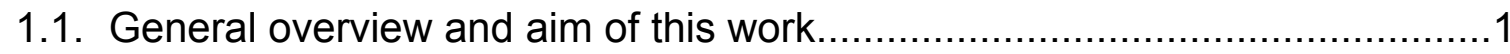

1.2. The hippocampus - a versatile structure in the CNS ...............................

1.3. Major functions of mitochondria in glial and neuronal cells ........................ 5

1.4. The strict demand for oxygen in the CNS ............................................

1.5. An overview of astrocytic function ................................................... 11

1.6. Influential factors on $\Delta \Psi_{\mathrm{m}}$ and mitochondrial structure ........................... 15

2. Materials and Methods .............................................................20

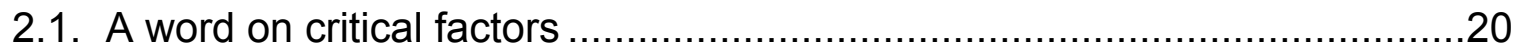

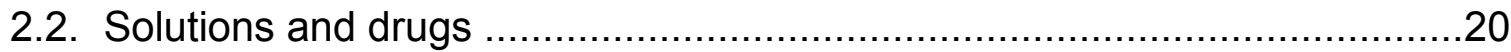

2.2.1. Artificial cerebrospinal fluid (ACSF) ........................................20

2.2.2. Cell culture medium ........................................................ 21

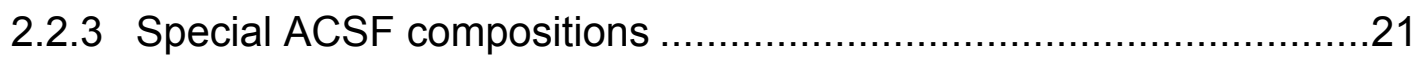

2.2.4. Drugs and chemical agents applied......................................22

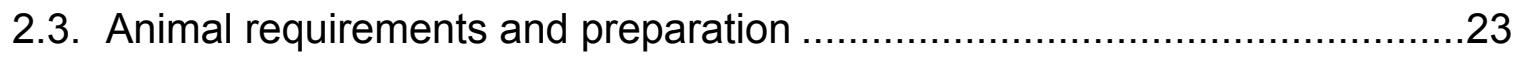

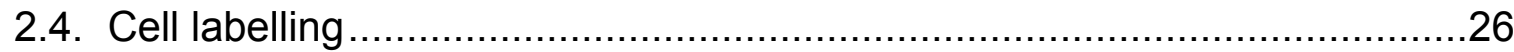

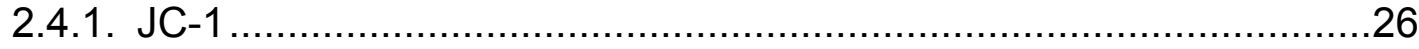

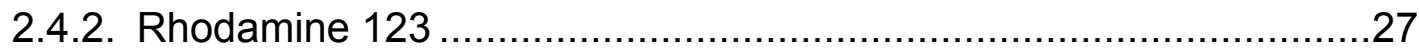

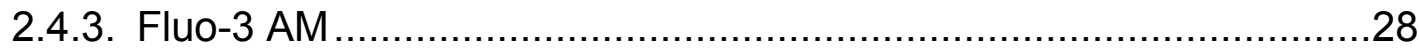

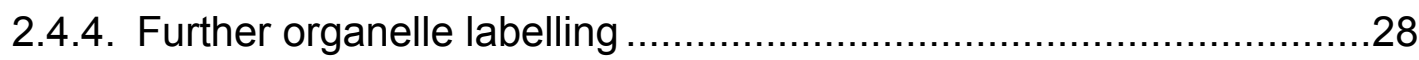

2.4.5. GFAP immunohistochemistry as an aid for cell identification ..........29

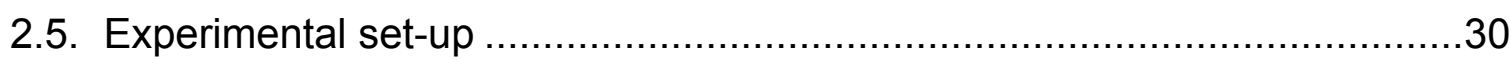

2.5.1. Set-up for both wide-field microscope and two-photon excitation laser imaging .....................................................

2.5.2. Optical microscope wide-field imaging ......................................31 
2.5.3. Two-photon excitation laser scanning microscopy …......................33

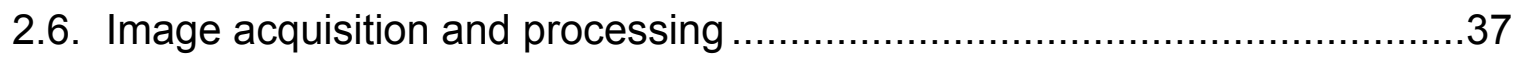

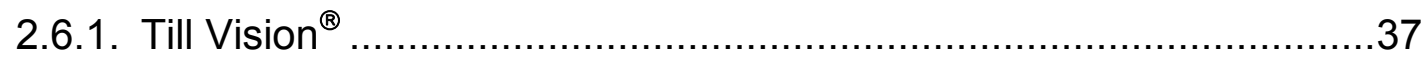

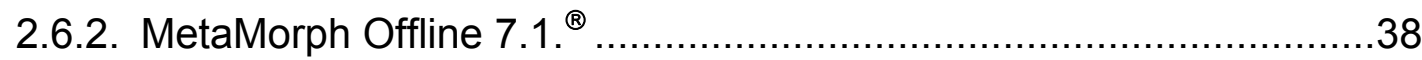

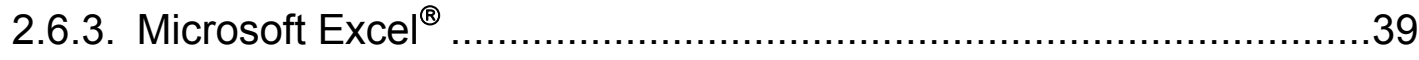

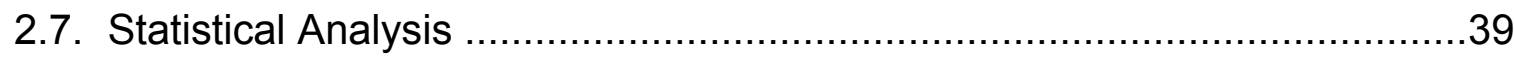

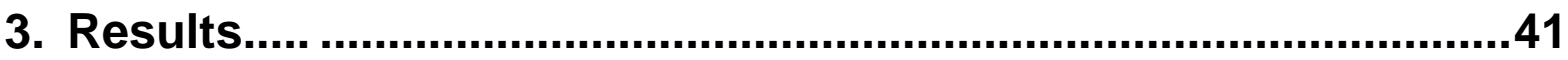

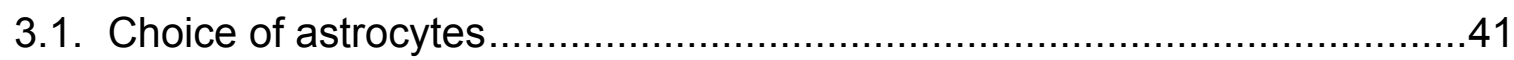

3.2. Morphological variety and flexibility of mitochondria ...............................42

3.2.1. Differences in mitochondrial shape...............................................

3.2.2. The subcellular distribution of mitochondria in astroglia ...................46

3.2.3. Colocalization of mitochondria with other organelles........................49

3.2.4. Observations of mitochondrial movement ………........................51

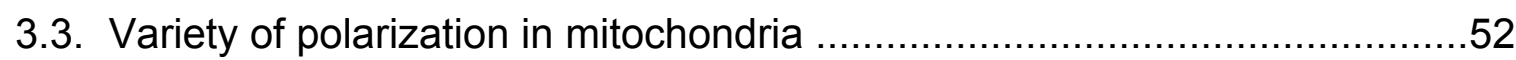

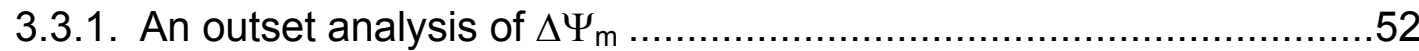

3.3.2. Analysis of polarization in respect of mitochondrial length ..............53

3.3.3. Analysis of polarization in respect of mitochondrial localization .......54

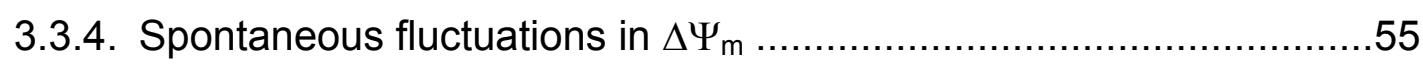

3.3.5. The influence of $\mathrm{Ca}^{2+}$ ions on $\Delta \Psi_{\mathrm{m}}$ transitions ..............................61

3.3.5.1. Nominally $\mathrm{Ca}^{2+}$ free ACSF in experiments .................................61

3.3.5.2. Dantrolene - a blocker of the ryanodine receptor ........................62

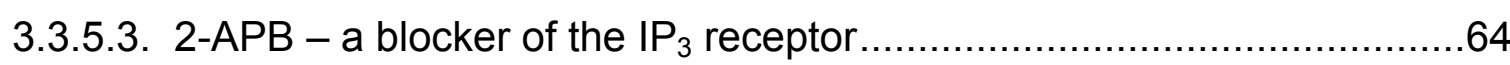

3.4. Effects of cellular $\mathrm{Ca}^{2+}$ load and metabolic impairment ............................65

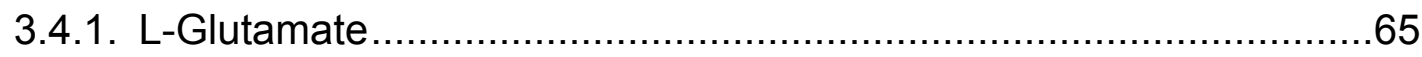

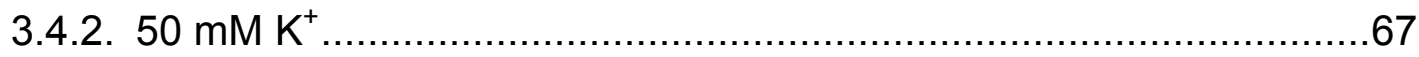

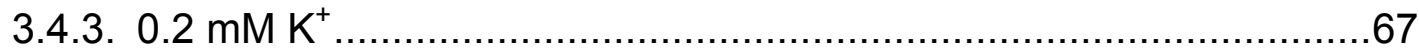

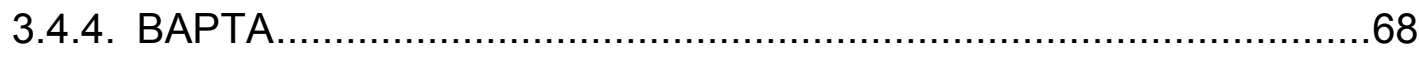

3.4.5. FCCP

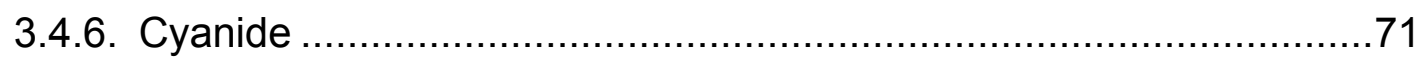




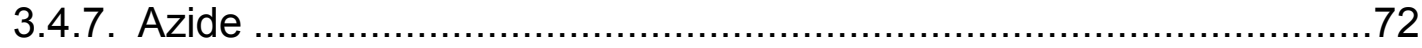

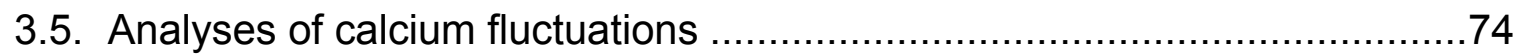

3.5.1. General aspects of $\mathrm{Ca}^{2+}$ transients in Fluo-3 labelled astrocytes .....74

3.5.2. Effects of dantrolene and 2-APB .................................................

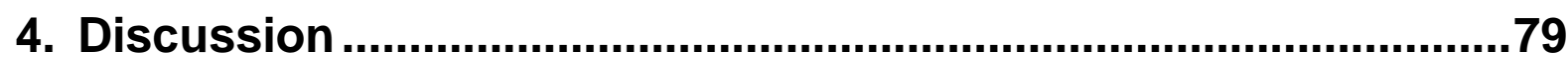

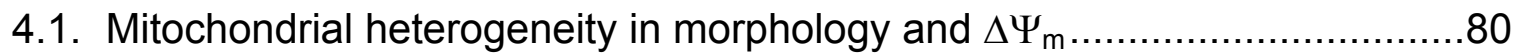

4.2. $\Delta \Psi_{\mathrm{m}}$ fluctuations - from analysis to interpretation ................................... 82

4.3. Heterogeneous reactions of mitochondria to metabolic challenge ..............86

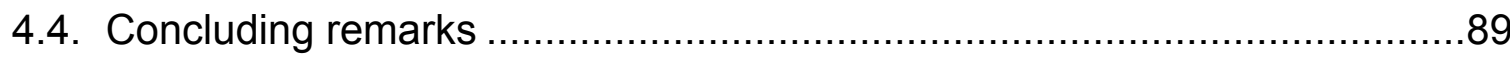

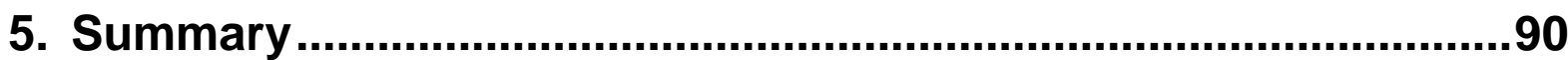

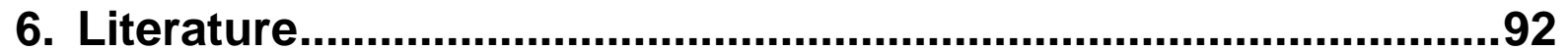

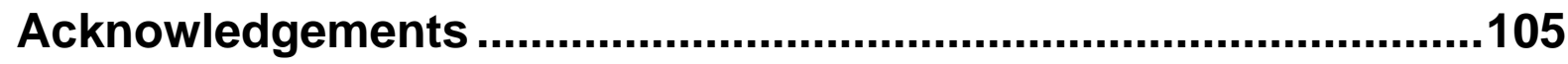

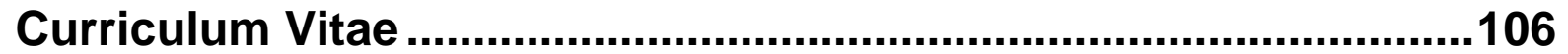




\section{Abbreviations}

\begin{tabular}{|c|c|}
\hline 2-APB & 2-Aminoethoxydiphenyl borate \\
\hline ACSF & artificial cerebrospinal fluid \\
\hline AMPA & a-Amino-3-hydroxy-5-methyl-4-isoxazolepropionic acid \\
\hline ATP & adenosine-5'-triphosphate \\
\hline BAPTA & 1,2-Bis(o-aminophenoxy)ethane- $\mathrm{N}, \mathrm{N}, \mathrm{N}^{\prime}, \mathrm{N}^{\prime}$-tetraacetic acid) \\
\hline $\mathrm{Ca}^{2+}$ & calcium \\
\hline CNS & central nervous system \\
\hline $\mathrm{CN}^{-}$ & cyanide \\
\hline DAPI & 4'-6-Diamidino-2-phenylindole \\
\hline DMSO & dimethyl sufoxide \\
\hline DIC & days in culture \\
\hline$\Delta \Psi_{\mathrm{m}}$ & mitochondrial transmembrane potential \\
\hline ER & endoplasmic reticulum \\
\hline $\mathrm{FADH}_{2}$ & flavin adenine dinucleotide \\
\hline FCCP & carbonyl cyanide 4-trifluoro-methoxyphenylhydrazone \\
\hline Fluo-3 & visible light calcium indicator \\
\hline GTP & guanidine triphosphate \\
\hline GFAP & glial fibrillary acidic protein \\
\hline $\mathrm{IP}_{3}$ & inositol triphosphate \\
\hline JC-1 & $\begin{array}{l}5,5^{\prime}, 6,6 \text { '-tetrachloro-1, 1',3,3'-tetraethylbenzimidazolyl- } \\
\text { carbocyanine iodide }\end{array}$ \\
\hline $\mathrm{K}^{+}$ & potassium \\
\hline $\mathrm{K}_{\mathrm{ir}}$ & inwardly rectifying potassium channel \\
\hline $\mathrm{NADH} / \mathrm{H}^{+}$ & nicotinamide adenine dinucleotide \\
\hline NMDA & N-methyl-D-aspartic acid \\
\hline $\mathrm{pO}_{2}$ & partial oxygen pressure \\
\hline Rho123 & rhodamine 123 \\
\hline $\mathrm{ROI}$ & region of interest \\
\hline ROS & reactive oxygen species \\
\hline RyR & ryanodine receptor \\
\hline TCA & tricarboxylic acid \\
\hline TPELSM & two-photon excitation laser scanning microscope \\
\hline UV & ultraviolet (light of $10-400 \mathrm{~nm}$ range) \\
\hline
\end{tabular}




\section{Introduction}

\subsection{General overview and aim of this work}

The role of mitochondria in neurological diseases evolves as an on-going focus in research. Meanwhile, a great variety of pathological conditions were proven to be manifestations of mitochondrial malfunction in the CNS (Leonard and Schapira 2000). For an even greater number of diseases impairment of neuronal mitochondria is a hypothetical aetiology or aggravating cofactor (Lin and Beal 2006); and arduous effort was made to illuminate how mitochondria influence the metabolism of neurones (Nicholls et al. 1999, Rego and Oliveira 2003).

Similar to the less investigated role of glial cells in neurodegeneration few publications concentrate on mitochondrial function in glia. Mitochondria have long been known to communicate pro- and anti-apoptotic signals within a cell (Kroemer and Reed 2000). In neuronal and glial tissue they were shown to significantly buffer and release calcium $\left(\mathrm{Ca}^{2+}\right)$ (Peuchen et al. 1996). $\mathrm{Ca}^{2+}$ acts as a crucial signalling molecule in some ways similar to a neurotransmitter (Clapham 2007). This aspect includes the role of $\mathrm{Ca}^{2+}$ in mitochondrial dysfunction and apoptosis as well as spreading depression and a link of mitochondria to intra- and intercellular $\mathrm{Ca}^{2+}$ fluctuations in neurones and astrocytes. In fact, it has been argued to suggest that astrocytes may even produce some kind of signalling on a basis of changes in subcellular ionic homeostasis and thus mitochondrial transmembrane potential $\left(\Delta \Psi_{\mathrm{m}}\right)$ (Ichas et al. 1997).

In 2001 astrocytes were reported to show fluctuations in the transmembrane potential of their mitochondria (Belousov et al. 2001). This phenomenon, first observed by Loew et al. in neuroblastoma cells (Loew et al. 1993), is also referred to as "blinking mitochondria" (Vergun et al. 2003). Later publications 
argued indecisively about the physiological nature of it and its possible link to apoptotic events inside the cell (Diaz et al. 1999). And despite of considerable effort already made in order to obtain sounder understanding of the mechanisms underlying these rhythmic $\Delta \Psi_{\mathrm{m}}$ fluctuations and their significance in vivo their molecular mechanism remains largely unclear (Vergun et al. 2003).

Furthermore researchers lately attempted to link subcellular morphologic aspects of neuronal cells to the clinical manifestation of a variety of neurodegenerative diseases (Trimmer et al. 2000, Baloyannis 2006). This not only includes Parkinson's or Alzheimer's disease but also morphologic changes or a pre-conditioning morphology of mitochondria in cells after stroke or other ischemic insults in nervous tissue (Schild et al. 2003). As loss of nervous tissue eventually occurs due to impaired cellular metabolism mitochondrial shape, distribution and $\Delta \Psi_{\mathrm{m}}$ have been a pronounced target of investigation. Here again neurones rather than glial cells were mostly in the focus.

Even fewer publications exist, that deal with the possible association of spatial distribution of mitochondria and their $\Delta \Psi_{\mathrm{m}}$ in glial cells during physiological conditions or under and after metabolic stress (Diaz et al. 1999). A particular focus of this thesis lies on a possible relation of $\Delta \Psi_{\mathrm{m}}$ and the morphology and distribution of the organelle within astrocytes as well as $\Delta \Psi_{\mathrm{m}}$ fluctuations and their dynamics in order to gain more clarification on physiological and morphological conditions, which either trigger or modify the phenomenon often referred to as "blinking mitochondria". This thesis thus aims at a greater understanding of mitochondrial heterogeneity in glial cells.

\subsection{The hippocampus - a versatile structure in the CNS}

The hippocampus was possibly given its name by the Italian anatomist G C Aranzi in the $16^{\text {th }}$ century referring to its stretched and wound up shape in the medial temporal lobe. It represents a locally eccentric and phylogenetically old 
part of the telencephalon - the archicortex. As a component of the limbic system the true role of the hippocampus was not functionally identified until the early $20^{\text {th }}$ century, when $\mathrm{V}$ Bekhterev noted anterograde amnesia in declarative memory in a patient with hippocampal impairment (Bechterew 1900). The hippocampus is vital to transfer working memory information to the short-term memory and stores facts and biographic information for some time until a consolidation sets in (Scoville and Milner 1957). Findings concerning hippocampal function were verified for rats as well as humans and explain why patients with hippocampal degradation suffer from variable amnesia and spatial disorientation as symptoms of dementia (Ekstrom et al. 2003).

A main aspect why the hippocampus is often the first structure to be affected in case of stroke or also degenrative dieseases is its selective vulnerability to oxygen deprivation and mitochondrial impairment (Donnelly et al. 1992, Davolio and Greenamyre 1995). Particularly CA1 neurons are most vulnerable. It was found that their synapse activity stops early upon oxygen deprivation (Cherubini et al. 1989). They probably even before ATP depletion first hyperpolarize due to potassium $\left(\mathrm{K}^{+}\right)$efflux and then depolarize (Erdemli et al. 1998, Hepp et al. 2005) and are likely to express channels that are sensitive to reactive oxygen species (ROS) (Lebuffe et al. 2003). Here mitochondria are the key structures. Their formation of ROS is not only elevated after hypoxic events (Perez-Pinzon et al. 1997) but also in neurodegenerative diseases such as Alzheimer's (Benzi and Moretti 1995). Still it is not clear whether this susceptibility of hippocampal neurons to hypoxia and ROS is caused by special predisposition of their mitochondria. However, astrocytes surrounding these vulnerable neurons play a vital part in maintaining ion homeostasis.

In 2004 the Rat Genome Sequencing Project Consortium announced a large sequencing similarity between the rat as a scientific tool and its great medical beneficiary $\mathrm{H}$. sapiens (RGSP Consortium 2004). It supports findings of similar anatomy of the hippocampus in many mammals and the rat in particular justifying the rat hippocampus as a long-established study object in medical 
research. Despite of some variability the order of the three cell layers forming the archicortex of the hippocampus remains largely the same (Amaral and Lavenex 2007). Outermost is the stratum oriens containing mainly basket cells, followed by a stratum of large glutamatergic pyramidal cells and a molecular stratum of mainly interneurones that link basket and pyramidal cells (Fig. 1). The density of large and clearly organized neurones as well as pronounced synaptic plasticity were some of the reasons why the hippocampus became such a popular object for electrical recordings and imaging as well as cell culture preparation. The anterior choroidal artery vascularizes the hippocampus and is an orientation mark for hippocampus preparation in the rat.

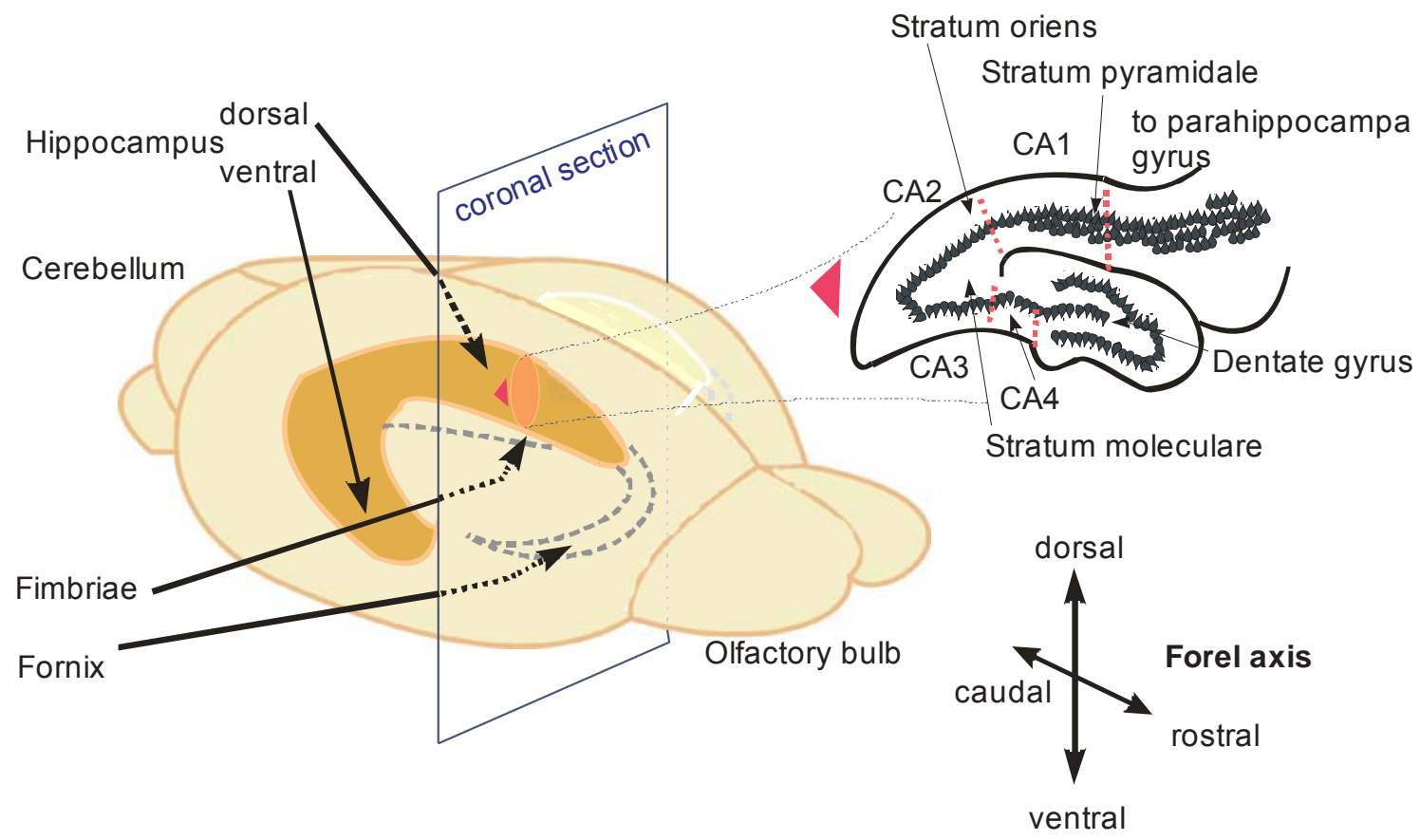

Figure 1 Anatomy of the rat hippocampus

The image depicts the brain of a neonatal rat used for cell culture preparation. The darker orange and light yellow regions mark the location of the banana-shaped hippocampi close to the lateral ventricles. The fornix is a closely attached structure of the limbic system. The detail gives a magnified impression of a coronal section as indicated in the overview: The hippocampus in an allocortical trilayer of three different strata and is divided into four main subfields (CA1-4). The dentate gyrus folds up at the medial side of the hippocampus formation. Compared to humans the rat hippocampus is in relation substantially larger.

Perpendicular to its allocortical trilayer the hippocampus is subdivided into the subfields $\mathrm{CA} 1^{1}, \mathrm{CA} 2, \mathrm{CA} 3$ and $\mathrm{CA} 4$, forming the central hippocampus, and adjacent structures named dentate gyrus and subiculum. The assembly is often

\footnotetext{
1 "CA" stands for "cornu ammonis", the "horn of the seahorse" - a synonym for the hippocampus.
} 
referred to as the hippocampus formation. Quite remarkably W Spielmeyer noted already in 1927 a selective vulnerability of the hippocampus to lack of oxygen (Spielmeyer 1927). Later analyses discovered neurones in the CA1 region as the most affected cells (Schmidt-Kastner and Freund 1991). While reasons for this vulnerability remain in the focus of research the hippocampus was for this reason established as a particularly apt object for hypoxia research leading to a variety of arterial occlusion models and in vitro schemes for hippocampal slices as well as cell cultures (Hossmann 2008). Since high vulnerability to metabolic compromise is related to mitochondrial function, hippocampal preparations are ideal study objects for the analysis of mitochondrial function and dysfunction.

\subsection{Major functions of mitochondria in glial and neuronal cells}

It is for their peculiar double membrane and the partial storage of their own DNA (mtDNA) that mitochondria by symbiosis are suspected to have become organelles of eukaryotes out of independent organisms a long time ago ${ }^{2}$ (Martin and Kowallik 1999). Without this serendipitous event life as it is would not be possible, for mitochondria are the site of oxidative phosphorylation and thus the main source of ATP production with some 28 molecules compared to only 4 ATP/GTP in glycolysis and the TCA cycle (Fig. 2). Accordingly mitochondrial dysfunction will lead to an ATP shortage of intolerable extent for neural tissue.

The four complexes granting oxidative phosphorylation are embedded in the layer of the inner mitochondrial membrane and transport electrons received from TCA cycle products as donors $\left(\mathrm{NADH} / \mathrm{H}^{+}\right.$and $\left.\mathrm{FADH} \mathrm{H}_{2}\right)$ which later react with oxygen and protons to $\mathrm{H}_{2} \mathrm{O}$ along the membrane via ubichinone and cytochrome c carriers.

\footnotetext{
${ }^{2}$ This theory is called the „endosymbiotic theory”.
} 


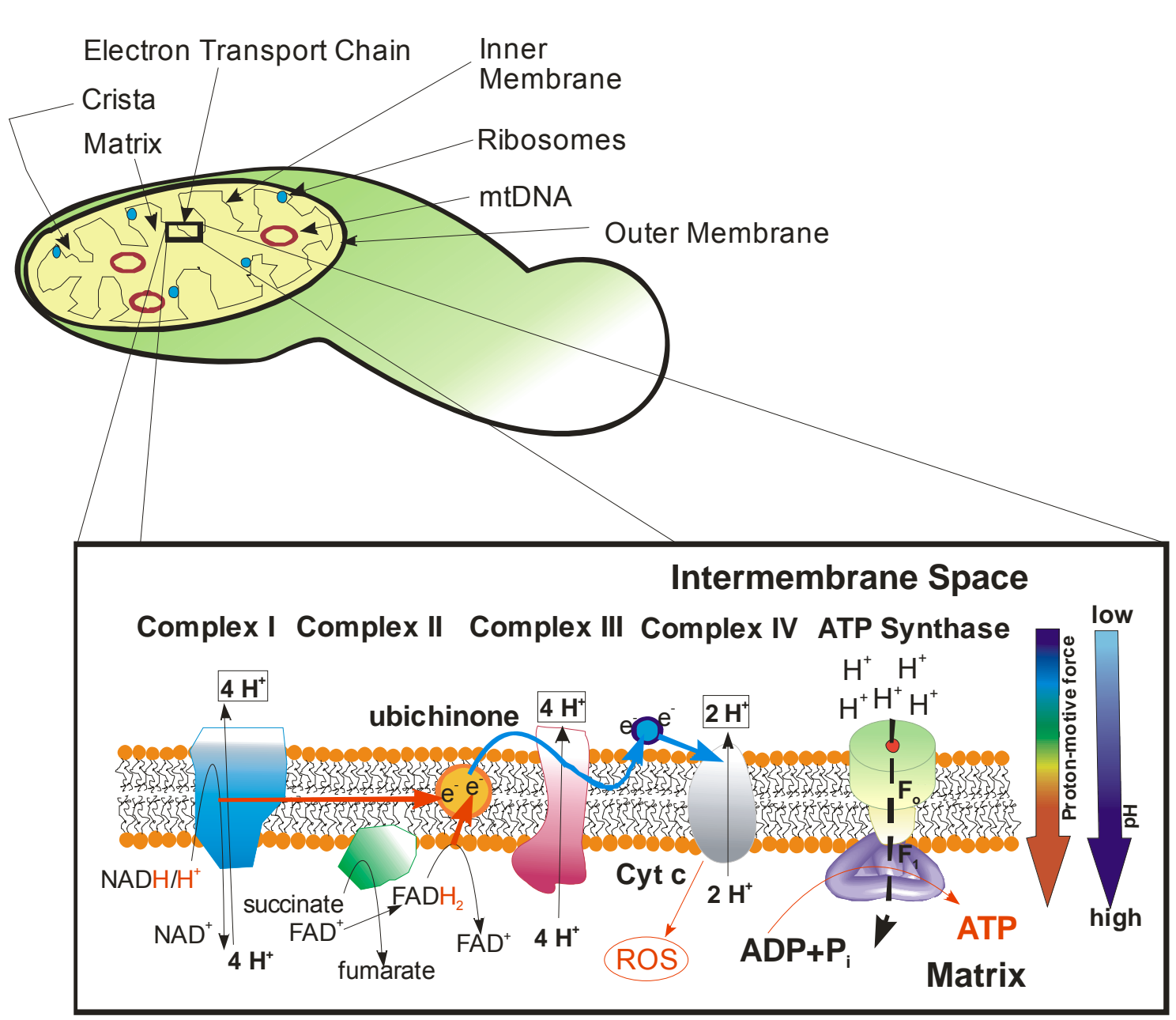

Figure 2 Mitochondrion and respiratory chain

The outer membrane of the mitochondrion has a typical eukaryotic composition, whereas the inner membrane - the site of oxidative phosphorylation - reveals the origin of mitochondria as independent organisms. The central lumen is called the matrix. TCA cycle products such as $\mathrm{NADH} / \mathrm{H}^{+}$or $\mathrm{FADH} \mathrm{H}_{2}$ are produced here and become substrates to complexes of the respiratory chain (see box). The matrix also contains the circular DNA of mitochondria. The lower schematic box illustrates how electrons won from substrate oxidation in complexes I and II are transported via ubichinone and cytochrome c. At complex III ubichinone is oxidized and electrons are passed over to cytochrome c. Finally all electrons react with oxygen and protons to water in complex IV (cytochrome c oxidase; detail not shown). The production of reactive oxygen species (ROS) by the complexes is a side effect of oxidative phosphorylation. The energy liberated in the oxidation reactions of complexes I, II and IV allows pumping protons against a chemical and electrical gradient resulting in a proton-motive force. This explains the origin of $\Delta \Psi_{\mathrm{m}}$. As protons reenter into the matrix via ATP synthase $\left(F_{0} F_{1}\right.$ complex) ATP is generated from ADP and phosphate by the enzyme.

The energy liberated in this redox reaction is used to pump protons from the mitochondrial matrix to the intermembrane space across the inner membrane from a compartment of relatively negative charge and low proton concentration to the intermembrane space - a positively charged region of already high proton concentration. The resulting proton-motive force provides the energy for ATP generation executed by the ATP synthase (or $F_{0} F_{1}$ ATPase or complex V). 
However, the generation of ATP has the production of ROS as a side effect.

It has been shown that this way of ATP production delivers at least $95 \%$ of the energy demand within the body (Erecinska and Silver 1989). Experiments with blockers of the complexes or uncouplers of the respiratory chain causing inhibition oxidative phosphorylation (for details (Foster et al. 2006)) or analyzing mitochondrial density as a marker for the activity of oxidative phosphorylation suggest that survival of neurones depends much more on oxidative ATP production than survival of glial cells such as astrocytes (Chen and Swanson 2003). Additionally, clinical findings in rat models of global cerebral hypoxia and in case studies of patients indicate, that oxygen dependence varies even between brain regions and individual neurones (Gibson et al. 1981, Ross and Graham 1993).

It seems that mitochondria at least in some cells are strategically located within cells paying tribute to local variations of ATP demand (Park et al. 2001, Müller et al. 2005). Mitochondria are transferred within a cell along microtubules. The stimuli for transportation might be local decline in ATP or accumulation of ROS species - both are signs, that already present mitochondria use their full capacity. Changed ADP and $\mathrm{Ca}^{2+}$ levels are similarly discussed (Yi et al. 2004, Mironov 2007). It is known that mitochondria are able to change shape and some research elucidated a correlation to metabolic activity or cell type with shape and clustering of mitochondria (Bereiter-Hahn and Voth 1994).

Mitochondrial polarization may vary within a cell or putatively even a single mitochondrion and correlates with the proton gradient built up in oxidative phosphorylation and hence the $\mathrm{pH}$ gradient. Active mitochondria in astrocyte show a high $\Delta \Psi_{\mathrm{m}}$ of about -145 mV or more (Diaz et al. 2000). This figure may be estimated by the Nernst equation. Today this activity can be visualized in living cells applying fluorescent probes, which change either the intensity or wavelength of light emission according to changes in $\Delta \Psi_{\mathrm{m}}$. 
Beyond the key role of ATP generation mitochondria are involved in $\mathrm{Ca}^{2+}$ sequestration from the cytosol of both neurones and astrocytes (Werth and Thayer 1994, Duchen 1999). In this context mitochondria have to be named as suspected mediators of apoptosis in neurones. Although it was argued that mild depolarization is cytoprotective due to lesser ROS production the greater impairment of complexes in the respiratory chain and resulting depolarization were experimentally linked to various diseases, e.g. Parkinson's disease for complex I malfunction (Votyakova and Reynolds 2001, Andrews et al. 2005).

Another fascinating aspect based upon the buffering capacity for $\mathrm{Ca}^{2+}$ of mitochondria is their influence on cytosolic $\mathrm{Ca}^{2+}$ concentration in astrocytes, whose modulation was suggested to play a vital part in signal transmission between neurones and astrocytes and has become a partial explanation for alteration of synaptic strength, e.g. in long term potentiation, or responses of neural networks to metabolic challenge as well as metabolic compromise (Haydon 2001).

\subsection{The strict demand for oxygen in the CNS}

It is tremendous that one fifth of the total oxygen supply to the human body is consumed by an organ weighing only $1.5 \%$ of the total body mass: the brain (Frosch et al. 2004). Due to the massive ATP consumption of neurones the brain highly depends on the oxidative phosphorylation of ATP rather than the less efficient glycolysis partially explaining its vulnerability to hypoxia.

Hypoxic conditions in the CNS may be classified in the following way. So-called functional hypoxia is a consequence of relatively low $\mathrm{pO}_{2}$ in the blood or impaired mitochondrial metabolisation of TCA cycle products, i.e. impairment of the respiratory chain. Reasons may be insufficient oxygen uptake by the lungs or mitochondrial intoxication by agents such as cyanide $\left(\mathrm{CN}^{-}\right)$or carbon monoxide $(\mathrm{CO})$ resulting in an impaired function of the respiratory chain. On the 
other hand there is the large group of ischemia-derived hypoxia in CNS tissue, where oxygen transport is the problem. It pathophysiologically may be subdivided into causes for global or focal cerebral stroke. Global ischemia has a systemic reason, such as shock, cardiac arrest or severe hypotension, whereas the total or subtotal occlusion of a cerebral artery results in focal ischemia. The clinical manifestation of the latter is more commonly known as stroke. Intracranial arteries are able to compensate a reduction of their lumen of up to $85 \%$ (Marx 2005). Beyond this point the oxygen extraction rate rises and cerebral blood flow drops from a physiological $50-80 \mathrm{ml} / 100 \mathrm{~g}$ tissue per minute to a critical $8 \mathrm{ml} / 100 \mathrm{~g} / \mathrm{min}$. and below. The latter figure acceptedly marks the threshold to irreversible ischemic tissue damage - oxygen extraction from the blood reaches its limit (Baron 2001).

Clinical research shows that cellular damage of the most vulnerable neurones reaches a point of no return after a period of only two to four minutes in total anoxia (Smith et al. 1984). Therefore not only the brain region depending on the occluded vessel, but also the time spent in hypoxic conditions has an impact on the severity and clinical consequences of the trauma. However, it was shown that cells in the CNS differ in their vulnerability to hypoxic conditions depending on cell type and brain region. Most vulnerable neural cell types are, besides CA1 neurones, cerebellar Purkinje cells and medium spiny striatal interneurones. Finally, in slowly evolving chronic hypoxia, such as for reasons of progressing atherosclerosis, it was suggested that the mammalian brain and in particular glial cells are able to adapt to some extent (Vega et al. 2006).

Both acute and chronic brain hypoxia mark substantial reasons for neurological impairment in Western society. According to the WHO ischemic stroke alone has become the second leading cause for mortality in the Western world (Bonita and Reddy 2003). Being on third rank as cause of death globally ischemic stroke can be perceived both as a reason for and an exacerbation of hypoxia in affected brain regions. Blood vessel occlusion and subsequent infarction will occur as a consequence of embolism, but often stroke derives from pathologic 
non-embolic changes in the vessel itself. These among others include atherosclerotic stenosis and hyalinisation of arteries leading to an impaired autoregulation of the vessel lumen with arterial hypertension and variable blood pressure being particular risk factors.

Haemodynamically caused absolute ischemia will result in an intolerable lack of ATP. Depolarization due to impaired function of the $\mathrm{Na}^{+} / \mathrm{K}^{+}$-ATPase will cause major $\mathrm{Ca}^{2+}$ influx through voltage-gated channels (see 1.5.). This activates cytolytic enzymes such as lipases and provokes further depolarization via intracellular pathways. Mitochondria are no longer able to buffer the cytosolic $\mathrm{Ca}^{2+}$ rise. The affected cells undergo apoptosis by opening of the mitochondrial permeability transition pore (MPTP). The boundaries of the affected brain region are determined by highly endangered tissue in a state of reactive hyperaemia following lactate accumulation, named the penumbra. Once the $\mathrm{O}_{2}$ perfusion is re-established ROS production rises in surviving cells causing a secondary damage which may excel the precedent hypoxic damage ${ }^{3}$ (Foster et al. 2006).

Transitory ischemic attacks (TIA) deriving from impaired tissue oxygenation were associated with a higher relative risk for dementia, another great issue for public health being enfaced in this century (Bos et al. 2007). A differentiated analysis of strategies for cell survival in all CNS cell types may one day hint at solutions to minimize neuronal loss in the actual patient suffering from neurodegeneration. The focus on mitochondria as main oxygen consumers during oxidative phosphorylation emerges as a vital aspect of research not only in terms of stroke, but comprises a wide range of hereditary and acquired neurodegenerative diseases (Beal 2005).

The term "vascular dementia" covers progressive cognitive impairment due to loss of neuronal tissue deriving from a large variety of reasons for vascular damage. It is often associated with Alzheimer's disease. The pathogenetic

\footnotetext{
${ }^{3}$ This complication is called the "reperfusion injury".
} 
model assumes that CNS tissue suffers from a permanent and yet variable hypoxic condition close to or sometimes at levels found in mini-strokes. Although some authors found examples of metabolic adaptation to relative hypoxia in neurones and glia in non-mammalian species, it is unlikely that human neurones can function unabatedly in an environment of intermittent oxygen shortage - a fact, which cumulates towards vascular dementia by an increased rate of neuronal apoptosis.

Astrocytes, however, show a greater capability to cope with elevated oxygen extraction rates and reduced ATP generation by oxidative phosphorylation than neurones and might even survive on glycolysis only. In comparative studies on long-term to short-term hypoxia astrocytes were shown to lower their ATP consumption after an initial activation of glycolysis (Vega et al. 2006). Indeed the consecutive elevation of lactic acid and protons in the CNS which is particularly high in diabetics is used as one possible explanation for the worse outcome of stroke in the hyperglycaemic patient referred to as the "glucose paradox". Furthermore analysis of mitochondrial shape and distribution of neurones kept in a hypoxic environment shows swelling and subsequent condensation of mitochondria (Solenski et al. 2002).

\subsection{An overview of astrocytic function}

After a long time of mistakenly being perceived as "neuronal glue" glial cells due to their complicated interaction with neurones have left this status in the past decades. Astrocytes outnumber neurones in the mammalian CNS by about 10 to 1 (Bignami 1991). Not only do they deliver mechanical stability to the neuronal network, but they also act as buffers for ions, neurotransmitters and metabolic products in the CNS network (Fig. 3). At the blood brain barrier astrocytes are in direct contact with capillaries and thus are the first recipients of glucose and oxygen for all cells in the CNS including neurones (Tsacopoulos and Magistretti 1996). In fact, astrocytes appear to be more vulnerable to 
acidosis than to lack of oxygen or hypoglycaemia.

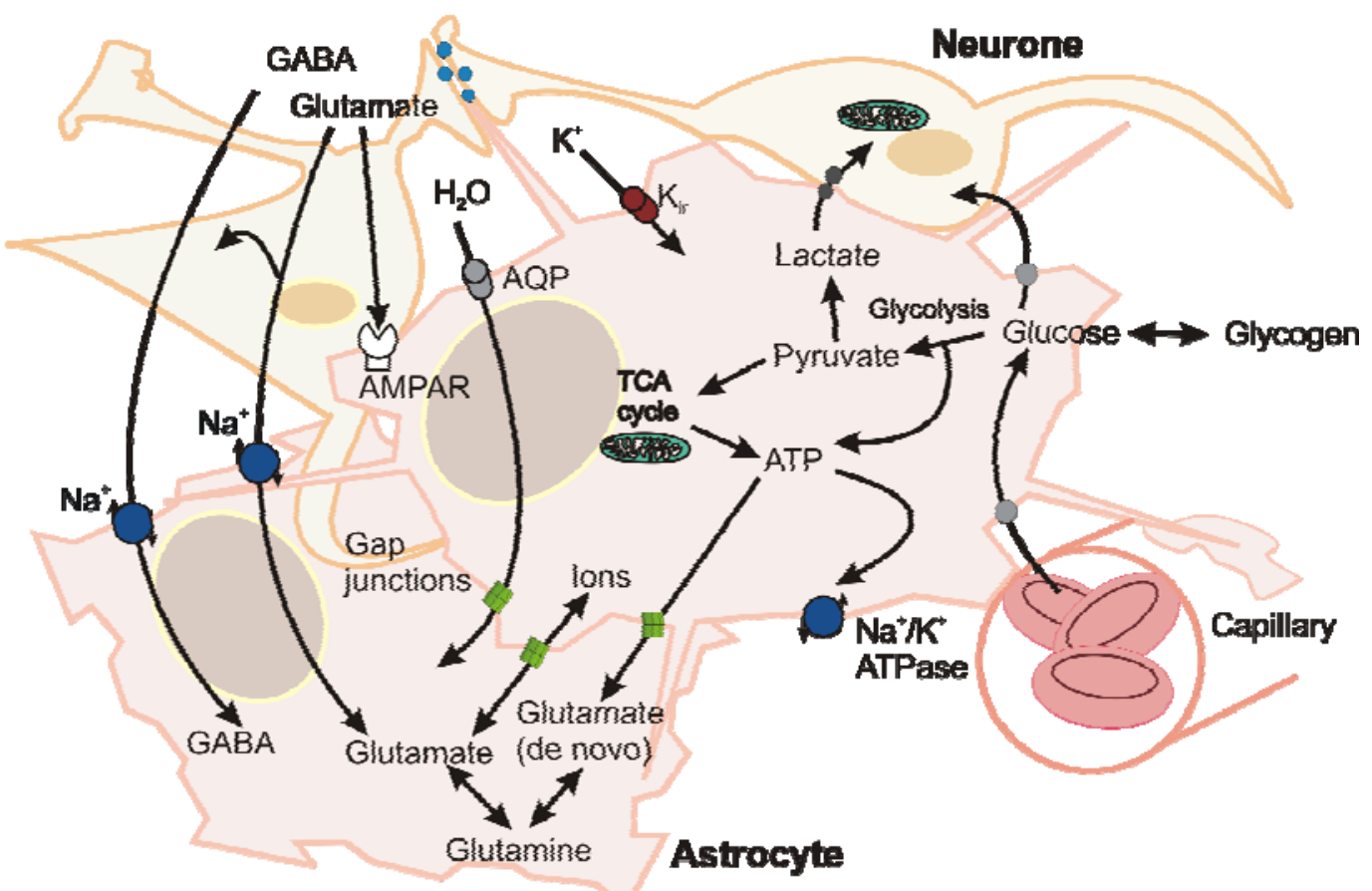

Figure 3 Astrocyte-Neurone interaction

The image can only provide a simplified overview. Main functions of astrocytes are (1) the uptake and storage of glucose, (2) the production of ATP for its own metabolism and surrounding neurones, (3) shuttling of lactate to neurones from glycolytically won pyruvate in case of oxygen deprivation, (4) uptake of neurotransmitters such as glutamate and GABA as well as (5) control of extracellular $\mathrm{K}^{+}$and $\mathrm{H}^{+}$levels and fluid transport via transporters, aquaporins and channels. Astrocytes can form a syncytium with gap junctions allowing cytosolic exchange of all these substances. The astrocyte surrounds the synapse and responds to chemical changes in the perisynaptic extracellular space. It has receptors for many neurotransmitters, e.g. AMPA receptors for glutamate. Changes in extracellular ion concentrations cause the opening of voltage-gated channels and alter the activity of transporters. The secondarily induced cascades inside the astrocyte cause the responsive extrusion of substances such as lactate, glutamate and $\mathrm{Ca}^{2+}$ into the synaptic cleft.

In the CNS astrocytes other than neurones exist as a functional syncytium. They are intensely coupled to each other via gap junctions made of astrocytespecific hemi-tunnels such as connexin 43 (Nadarajah et al. 1996). This means that their cytosol is in direct contact allowing a passage of ions, glucose, neurotransmitters and fluid over large distances. In this sense astrocytes, for example, deliver lactate as a metabolite for the TCA cycle to active neurones and remove extruded $\mathrm{K}^{+}$from these acting as a neuroprotective buffer. A main channel involved in the $\mathrm{K}^{+}$uptake is the ATP dependent $\mathrm{K}_{\mathrm{ir}} 4.1$ (KCNJ 10) which develops $\mathrm{Ca}^{2+}$ permeability in a low $\mathrm{K}^{+}$extracellular environment (Dallwig et al. 2000, Butt and Kalsi 2006, Hartel et al. 2007). 
Similarly, astrocytes protect neurones from excitotoxicity of excessive glutamate and GABA via removal from the synaptic cleft across specific $\mathrm{Na}^{+}$dependent transporters. On the other hand astrocytes store glutamate in order to pass it back to neurones as soon as it is needed and participate in de novo glutamate synthesis (Hertz et al. 1999, Parpura et al. 2004). Furthermore they were shown to produce neurotrophins such as BDNF and modulate inflammatory response in the CNS (Dougherty et al. 2000, Wu et al. 2004). From this perspective it is not surprising that a role of astrocytes as neuromodulators in synaptic plasticity was proposed. It was found that astrocytes can change the excitability of neurones by modulation of extracellular potassium and glutamate or of neuronal $\mathrm{Ca}^{2+}$ (Ullian et al. 2001, Magistretti 2006). Therefore recent concepts of synapse function and plasticity include astrocytes. Nedergaard et al. argued that glia may even be physically linked to neurones via gap junctions (Nedergaard 1994). Astrocytes may also respond to local deficiencies of oxygen via induction of angiogenesis and modulation of arteriolar width. Astrocytic $\mathrm{Ca}^{2+}$ waves appear to be part of the physiological mechanisms involved (Sinor et al. 1998, Gordon et al. 2008).

Since communication is not unilateral these examples illustrate vice versa the impact of neuronal activity on subcellular processes inside the astrocytes. $\mathrm{Ca}^{2+}$ homeostasis inside the cell gives one good example of the complexity of interaction between different organelles and channels. Among other pathways $\mathrm{Ca}^{2+}$ may enter astrocytes via voltage-gated channels (VGCC). Apparently these are only expressed in the presence of neurones and open upon depolarization of the astrocyte cell membrane as seen during neuronal activity when extracellular $\mathrm{K}^{+}$is high (Verkhratsky and Kettenmann 1996, Verkhratsky et al. 1998).

High glutamate concentrations may also elevate $\mathrm{Ca}^{2+}$ in at least two ways: by ionotropic AMPA receptors, that trigger depolarization via cationic ion influx and via G-protein coupled metabotropic receptors that lead to a rise in $\mathrm{IP}_{3} . \mathrm{IP}_{3}$ has receptors on the ER of the astrocyte causing $\mathrm{Ca}^{2+}$ release into the cytosol. $\mathrm{Ca}^{2+}$ 
may also be released from the ER via channels of the ryanodine receptor (RyR) - $\mathrm{Ca}^{2+}$ sensitive $\mathrm{Ca}^{2+}$ channel that represents the model of $\mathrm{Ca}^{2+}$ induced $\mathrm{Ca}^{2+}$ release in astrocytes $(\mathrm{CICR})$. Under physiological conditions the astrocyte can re-balance its homeostasis of the ion by ATP dependent transport either across the cell membrane or by intracellular buffering of $\mathrm{Ca}^{2+}$, e.g. by sequestration in the endoplasmic reticulum (ER) via the $\mathrm{Ca}^{2+}$ ATPase of the organelle - SERCA (Mata and Fink 1989, Kostyuk and Verkhratsky 1994) or in mitochondria.

Storage of ions and neurotransmitters locally transported into the astrocyte, e.g. at the synaptic cleft, can result in unequal concentrations within the syncytium a concentration gradient builds up. For $\mathrm{Ca}^{2+}$ ions a wavelike cytosolic propagation was described in astrocyte cultures (Cornell-Bell et al. 1990). They can be triggered by mechanical manipulation, low extracellular $\mathrm{Ca}^{2+}$ concentration, UV light and glutamate (Verkhratsky and Kettenmann 1996). For glutamate extracellular $\mathrm{Ca}^{2+}$ is apparently needed for propagation. Quite interestingly the waves vary both in frequency of oscillations and in velocity of propagation with ranges from 5 to $20 \mu \mathrm{m} / \mathrm{s}$ or more described (Charles et al. 1991). Both the mechanism and physiological nature of glial $\mathrm{Ca}^{2+}$ waves are issues still argued about. As regard to the mechanism extracellular stimuli such as ATP as well as cytosolically transported mediators of $\mathrm{Ca}^{2+}$ elevation such as $\mathrm{IP}_{3}$ both play their part in $\mathrm{Ca}^{2+}$ wave generation and propagation (Finkbeiner 1992, Guthrie et al. 1999). The inhibition of $\mathrm{IP}_{3}$ mediated $\mathrm{Ca}^{2+}$ release was found to suppress the propagation of $\mathrm{Ca}^{2+}$ waves (Charles et al. 1993). Possible physiological roles nowadays involve their regular participation in the modulation of neurotransmitter and ion concentration in neurones and conclusively a modulatory function in CNS architecture (Hansson and Ronnback 2003, Fellin and Carmignoto 2004, Allen and Barres 2005). However, $\mathrm{Ca}^{2+}$ transients are also supposed to be involved spreading depression, ischemic cell death and epilepsy (Basarsky et al. 1998, Takano et al. 2009). Only some years ago there was evidence found for $\mathrm{Ca}^{2+}$ waves to occur not only in cell cultures but also in intact brain tissue which raises hopes to be able to integrate $\mathrm{Ca}^{2+}$ waves in models of in-vivo brain function (Hirase et al. 2004). 
The primary hippocampal cell cultures used for my experiments did not exclusively consist of glia. Generally astrocytes can easily be distinguished from neurons due to their larger size - meaning the soma in particular. They are not as filigran, but of a rather amoeboid shape and flat. Individual mitochondria can better be observed in glia due to their lesser density. It is also interesting to know that not only different kinds of glia but also different subpopulations of astrocytes vary in their expression of receptors and channels - a fact that may have pivotal influence on $\Delta \Psi_{\mathrm{m}}$ (Porter and McCarthy 1997). However, to prove that the used cell cultures predominantly contained glia and in order to show the variety of glial appearance GFAP immunolabeled astrocytes were imaged. For experiments only cytoplasmic and not stellate-shaped glia was thought eligible in order to minimize the number of oligodendrocytes recorded.

\subsection{Influential factors on $\Delta \Psi_{\mathrm{m}}$ and mitochondrial structure}

Mitochondria at rest in various species were found to have a basic $\Delta \Psi_{\mathrm{m}}$ of about -180 to $-150 \mathrm{mV}$ (Mitchell 1966). However, deviations of this state were registered in astrocytes under different conditions (Loew et al. 1993, Diaz et al. 2000). Primarily mitochondrial polarization depends on the proton pumping activity of their respiratory chain keeping up the voltage between the matrix and the intermembrane space. Possible modulators besides the availability of oxygen are the actual ATP demand of the cell and changes in cytosolic and mitochondrial ion homeostasis.

It was long doubted that mitochondria substantially function as $\mathrm{Ca}^{2+}$ stores. However, experiments have shown that mitochondria in astrocytes at rest seem to buffer large amounts of $\mathrm{Ca}^{2+}$ as long as they are sufficiently polarized (Nicholls 1985). It was proposed that mitochondria can accumulate $250-1000$ $\mathrm{nM}$ of $\mathrm{Ca}^{2+}$ depending on a sufficiently high rise in cytosolic $\mathrm{Ca}^{2+}$. Cytosolic $\mathrm{Ca}^{2+}$ was estimated only 100nm at rest (Walz and Wilson 1986). Finkbeiner et al. proclaimed a possible rise in cytosolic $\mathrm{Ca}^{2+}$ from intracellular stores to $2 \mu \mathrm{M}$ 
under certain circumstances such as elevated extracellular glutamate levels (Cornell-Bell et al. 1990, Finkbeiner 1993). Metabotropic glutamate receptors elevate cytosolic $\mathrm{IP}_{3}$ levels. This intracellular messenger was shown to cause $\mathrm{Ca}^{2+}$ accumulation in mitochondria following local rises in cytosolic $\mathrm{Ca}^{2+}$ probably by $\mathrm{IP}_{3}$ triggered $\mathrm{Ca}^{2+}$ release from the ER (Rizzuto et al. 1993). The suggested uptake mechanism is a fast $\mathrm{Ca}^{2+}$ uniporter ATPase which has a high affinity to $\mathrm{Ca}^{2+}$ as well as a passive uptake driven by the mitochondrial electrochemical gradient (Gunter and Pfeiffer 1990, Kirichok et al. 2004) (Fig. 4). In some way this mitochondrial uniporter competitively buffers elevations of cytosolic $\mathrm{Ca}^{2+}$ with SERCA, the Ca ${ }^{2+}$ ATPase of (Pozzo-Miller et al. 1997) the partially colocalized ER. Indeed in experiments with thapsigargin, a blocker to SERCA, an elevation in mitochondrial $\mathrm{Ca}^{2+}$ uptake beyond average was found (Pozzo-Miller et al. 1997). The ER and mitochondria seem to develop a synchronized time mode for $\mathrm{Ca}^{2+}$ uptake and release (Pivovarova et al. 2002). Mitochondrially stored $\mathrm{Ca}^{2+}$ is released by a $\mathrm{Na}^{+} / \mathrm{Ca}^{2+}$ antiporter whose transport capacity is 30 times slower than of the uptaking ATPase (Babcock et al. 1997). By these mechanisms both mitochondria and the ER are presumed to modulate and synchronize wavelike fluctuations in cytosolic $\mathrm{Ca}^{2+}$. Jouaville et al. on Xenopus oocytes suggest a voltage-dependent uptake in mitochondria and as a consequence fluctuating cytosolic $\mathrm{Ca}^{2+}$ levels that alter $\mathrm{IP}_{3}$ induced $\mathrm{Ca}^{2+}$ release due to a decreased opening probability of ER $\mathrm{Ca}^{2+}$ channels (Jouaville et al. 1995). For neuronal mitochondria in squid presynaptic terminals variable buffering capacity for $\mathrm{Ca}^{2+}$ was supposed to be an element in synaptic plasticity (Jonas et al. 1999). 


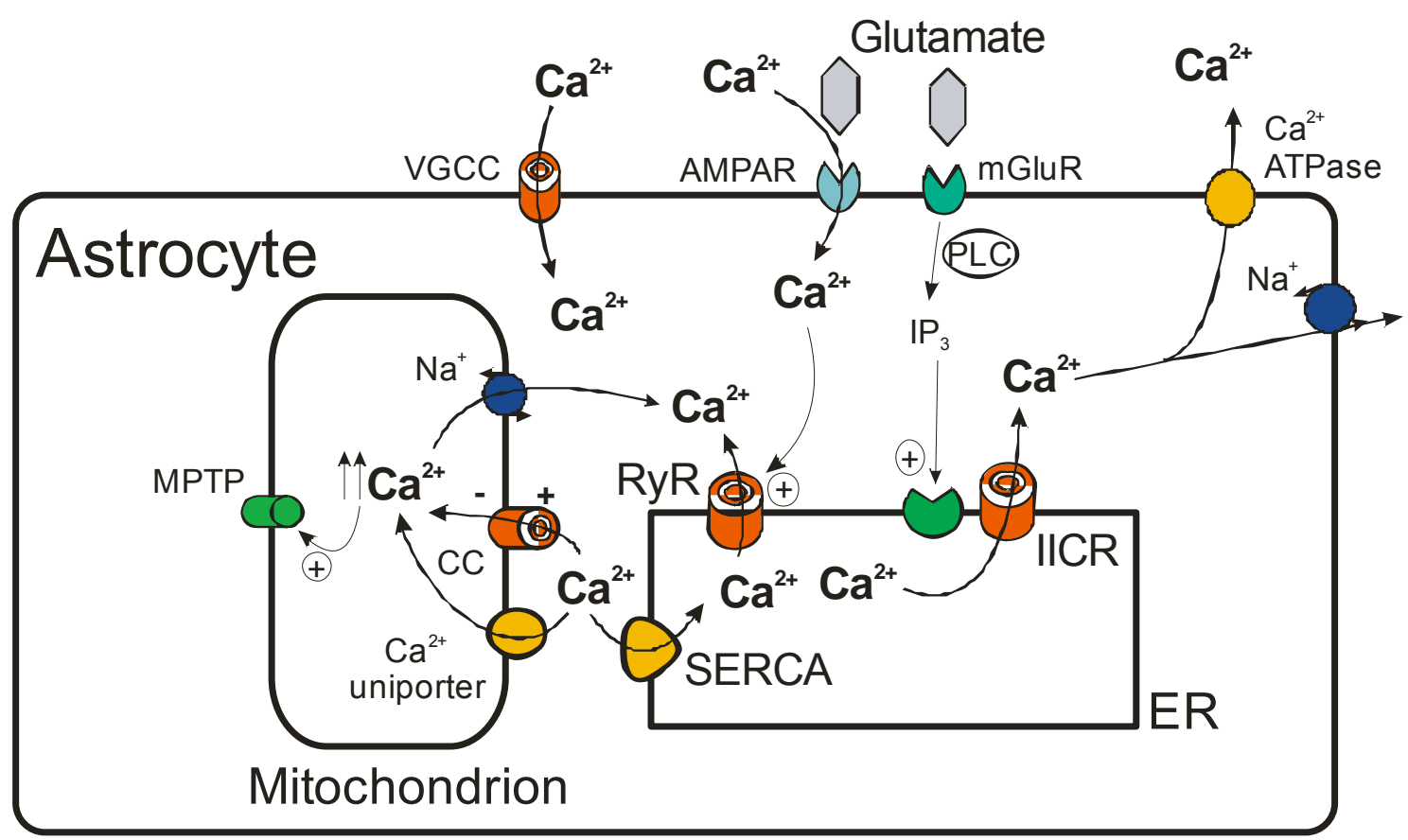

Figure 4 Intracellular $\mathrm{Ca}^{2+}$ homeostasis and organelle interaction

This is a simplified overview. $\mathrm{Ca}^{2+}$ enters the astrocyte, e.g., via voltage-gated channels (VGCC) or ionotropic glutamate receptors (e.g. AMPAR) that contain a non-selective cation channel. Metabotropic glutamate receptors (mGluR) elevate the concentration of $\mathrm{IP}_{3}$ via phospholipase $\mathrm{C}$ activation $(\mathrm{PLC}$ ). The messenger stimulates $\mathrm{Ca}^{2+}$ efflux from the ER binding at IP 3 receptors (IICR $-\mathrm{IP}_{3}$ induced $\mathrm{Ca}^{2+}$ release). $\mathrm{Ca}^{2+}$ may also leave the ER via ryanodine sensitive $\mathrm{Ca}^{2+}$ channels/receptors (RyR) that open at elevated cytosolic $\mathrm{Ca}^{2+}$ levels (CICR $-\mathrm{Ca}^{2+}$ induced $\mathrm{Ca}^{2+}$ release). The ER accumulates cytosolic $\mathrm{Ca}^{2+}$ via SERCA - its Ca2+ ATPase. SERCA acts competitively to the mitochondrial $\mathrm{Ca}^{2+}$ uniporter. Another way of mitochondrial $\mathrm{Ca}^{2+}$ accumulation probably is via influx of the cation $\mathrm{Ca}^{2+}$ via capacitive channels (CC) depending on mitochondrial $\Delta \Psi_{\mathrm{m}}$. If mitochondrial $\mathrm{Ca}^{2+}$ exceeds a certain concentration the ion may cause opening of the mitochondrial permeability transition pore (MPTP) - a factor in early apoptosis. Mitochondria can raise cytosolic $\mathrm{Ca}^{2+}$ via a $\mathrm{Na}^{+} / \mathrm{Ca}^{2+}$ antiporter. Similar to the $\mathrm{Na}^{+} / \mathrm{Ca}^{2+}$ antiporter of the cell membrane it can probably work also in reverse mode causing an influx. Cytosolic $\mathrm{Ca}^{2+}$ can additionally be extruded from the astrocyte via another $\mathrm{Ca}^{2+}$ ATPase.

The metabolic and buffering activity of mitochondria has an influence on the morphology of the organelle which can easily be visualized in astrocytes due to their relatively lesser density of mitochondria than in other cell types such as neurones. ATP depletion was observed to make mitochondria wound up, swollen and cup-shaped (Bereiter-Hahn and Voth 1994). Swollen mitochondria were also regularly observed in brain preparations of brains of patients with Parkinson's (Christie-Pope et al. 1989). In 2005 Müller et al. showed that FCCP, which uncouples mitochondria and dissipates $\Delta \Psi_{\mathrm{m}}$, blocks mitochondrial movement in the cytosol probably due to the dependence of microtubules on a local ATP delivery by the transported mitochondrion (Müller et al. 2005). Besides this transport an active motion by local swelling and shrinking 
according to altered ATP/ADP quotients is discussed (Bereiter-Hahn et al. 1983, Loew et al. 1993).

Another aspect of mitochondria is their ability to fuse and divide (fission). This is peculiar because it suggests that mitochondria share the same genes inside one cell and still show differences, e.g. in $\Delta \Psi_{\mathrm{m}}$ depending on their cytosolic location (Smiley et al. 1991). A colocalization of mitochondria and the ER had often been observed. Müller et al. described also the formation of dense mitochondrial clusters for neuronal mitochondria (Bereiter-Hahn and Voth 1994; Müller 2005). This observation motivated scientists to focus on functional mitochondrial heterogeneity as e.g. as can be defined by localization of the mitochondrion inside the cell. Other groups focussed on a phenomenon of transient changes in $\Delta \Psi_{\mathrm{m}}$ - often described as oscillations, blinking, fluctuations or flickers. Studies on both isolated as well as in-situ mitochondria of different species exist (Duchen et al. 1998, Diaz et al. 2000, Vergun and Reynolds 2004). Some groups estimated changes in mitochondrial polarization applying the Nernst equation (Scaduto and Grotyohann 1999, Diaz et al. 2000). O'Reilly et al. observed in toad gastric muscle cells that $83 \%$ of the mitochondria spontaneously showed reversible, transient depolarizations (O'Reilly et al. 2003). However, the assumption that one mitochondrion may show alternating different polarizations at the same time (Smiley et al. 1991) contradicts experiments supporting the "cable theory" of mitochondria showing a total depolarization of the entire organelle after only partial laser radiation (Siemens et al. 1982, Skulachev 2001, Müller et al. 2005).

Another absorbing aspect is the mechanism behind the fluctuations. Several groups experimented with the effect of $\mathrm{Ca}^{2+}$ loads on $\Delta \Psi_{\mathrm{m}}$. Vergun et al. found that a low ATPIADP quotient as well as high $\mathrm{Ca}^{2+}$ loads stop $\mathrm{Ca}^{2+}$ induced fluctuations in isolated brain mitochondria due to depolarization (Vergun and Reynolds 2004). This result is coherent with a publication describing depolarizations after glutamate or FCCP induced $\mathrm{Ca}^{2+}$ accumulations in mitochondria of hippocampal neurones (Bindokas et al. 1998). Moderate $\mathrm{Ca}^{2+}$ 
elevation in mitochondria was, however, not sufficient to depolarize the organelles in astrocytes (Kahlert et al. 2001). In this context the still undetermined interpretation of the physiological nature of fluctuations in $\Delta \Psi_{\mathrm{m}}$ needs to be mentioned. Neither were the underlying molecular mechanisms ever revealed, nor was an analysis of mitochondrial heterogeneity concerning the phenomenon as regard to location, shape or average $\Delta \Psi_{\mathrm{m}}$ seriously attempted. Arguing that fluctuations in $\Delta \Psi_{\mathrm{m}}$ are $\mathrm{Ca}^{2+}$ dependent apparently only high $\mathrm{Ca}^{2+}$ concentrations beyond the level at which fluctuations in $\Delta \Psi_{\mathrm{m}}$ occur trigger the transient opening of the mitochondrial permeability transition pore complex - a multiprotein complex that plays a pivotal role in apoptosis. Experiments with blockers as well as agonists of the pore delivered variable results that may contradict a substantial involvement of the pore in the development of fluctuations and support a physiological role (Vergun et al. 2003). 


\section{Materials and Methods}

The following sections will give a detailed overview of all techniques applied and the instrumentations used for the experimental approach of this thesis.

\subsection{A word on critical factors}

Research on living cells requires care in terms of preserving not only cellular viability during the experiment but also a physiological environment for natural acting of the cell as far as this is possible. When applying imaging techniques critical factors that may impair this prerequisite are the toxicity of fluorescent probes and exposure to UV radiation during the experiment. Therefore test experiments were performed to identify minimum doses necessary for fluorescent dyes, drugs and light exposure times. The concentration of DMSO as a solvent was always kept below $0.2 \%$ in the cell medium to avoid possible side effects.

Animal killing for organ extraction in science such as for cell culture preparation was announced and registered ${ }^{4}$. No experiments were performed in living animals. All primary cell cultures were used readily to minimise waste and animal material.

\subsection{Solutions and drugs}

Unless otherwise mentioned all chemicals were purchased from Sigma-Aldrich ${ }^{\circledR}$.

2.2.1. Artificial cerebrospinal fluid (ACSF)

ACSF had the following composition (in mM): $130 \mathrm{NaCl}, 24 \mathrm{NaHCO}_{3}, 10$

${ }^{4}$ Mitteilung zur Tötung von Wirbeltieren T-31/03 und T-13/08 (Tierschutzbeauftragter der Univ. Göttingen). 
Dextrose, $3.5 \mathrm{KCl}, 1.25 \mathrm{NaH}_{2} \mathrm{PO}_{4}, 1.2 \mathrm{CaCl}_{2}$ and $1.2 \mathrm{MgSO}_{4}$. A physiological $\mathrm{pH}$ of 7.4 was maintained by keeping the ACSF at a constant temperature of 38 ${ }^{\circ} \mathrm{C}$ while aerating constantly with $95 \% \mathrm{O}_{2}-5 \% \mathrm{CO}_{2}$ (carbogen). Some experiments were run applying in low or high $\mathrm{KCl}(0.2 \mathrm{mM}$ or $50 \mathrm{mM})$ or nominally $\mathrm{Ca}^{2+}$ free ACSF for investigatory questions (see below).

\subsubsection{Cell culture medium}

Cell culture medium consisted of a minimal essential medium (MEM, by Invitrogen) with $5 \mathrm{~g}$ of supplemented glucose, $200 \mathrm{mg} \mathrm{NaHCO} 3$ and $100 \mathrm{mg}$ transferrin $\left(\right.$ Calbiochem ${ }^{\circledR}$ ) per litre. To produce plating medium this base medium was supplemented with FBS $10 \%$ (fetal bovine serum; Biochrom ${ }^{\circledR}$ ), 2 $\mathrm{mM} \mathrm{L-glutamine} \mathrm{and} 25 \mu \mathrm{l} / \mathrm{ml}$ insulin. A day after preparation plating medium was exchanged by growth medium. This contains FBS 5\%, $0.5 \mathrm{mM}$ L-glutamine, $20 \mu \mathrm{l} / \mathrm{ml} \mathrm{B}-27$ as a growth factor $\left(\right.$ Invitroge ${ }^{\circledR}$ ) and $100 \mu \mathrm{g} / \mathrm{ml}$ penicillinstreptomycin $\left(\right.$ Biochrom $^{\circledR}$ ) to prevent microbial contamination.

\subsubsection{Special ACSF compositions}

Apart from standard ACSF solutions with $50 \mathrm{mM}$ potassium, $0.2 \mathrm{mM}$ potassium and nominally no $\mathrm{Ca}^{2+}$ were applied. $50 \mathrm{mM}$ of $\mathrm{K}^{+}$in the extracellular compartment depolarize astrocytes causing substantial $\mathrm{Ca}^{2+}$ influx into the cell via activation of voltage-gated $\mathrm{Ca}^{2+}$ channels (VGCC).

Exposure to $0.2 \mathrm{mM} \mathrm{K}^{+}$ACSF for several minutes instead of a regular ACSF containing $3.5 \mathrm{mM} \mathrm{K}^{+}$triggers $\mathrm{Ca}^{2+}$ influx into the cytosol of astrocytes via then $\mathrm{Ca}^{2+}$ permeant $\mathrm{K}_{\text {ir }}$ channels. Nominally $\mathrm{Ca}^{2+}$ free ACSF restricts the possible sources of released $\mathrm{Ca}^{2+}$ to intracellular stores. During the recording the cell culture usually was first placed in ACSF of $1.2 \mathrm{mM} \mathrm{Ca}^{2+}$ and then exposed to $\mathrm{Ca}^{2+}$ free ASCF prior to drug application. 
2.2.4. Drugs and chemical agents applied

\begin{tabular}{|c|c|c|c|c|}
\hline $\begin{array}{l}\text { Drug/ Agent } \\
\text { Full name }\end{array}$ & $\begin{array}{c}\text { Stock } \\
\text { solution }\end{array}$ & Storage & $\begin{array}{c}\text { Final } \\
\text { concentration }\end{array}$ & $\begin{array}{l}\text { Mode of } \\
\text { action }\end{array}$ \\
\hline $\begin{array}{l}\text { 2-APB } \\
\text { 2-Aminoethoxydiphenylbo- } \\
\text { rate }\end{array}$ & $\begin{array}{l}100 \mathrm{mM} \text { in } \\
\text { DMSO }\end{array}$ & $-18^{\circ} \mathrm{C}$ & 10 or $100 \mu \mathrm{M}$ & $\begin{array}{l}\mathrm{IP}_{3} \text { receptor } \\
\text { blocker } \\
(\mathrm{ER})\end{array}$ \\
\hline $\begin{array}{l}\text { Azide } \\
\mathrm{NaN}_{3}\end{array}$ & $\begin{array}{l}1 \mathrm{M} \text { in } \mathrm{H}_{2} \mathrm{O} \\
\text { destillata }\end{array}$ & $-18^{\circ} \mathrm{C}$ & 2 or $4 \mathrm{mM}$ & $\begin{array}{l}\text { Inhibitor of } \\
\text { complex IV of } \\
\text { the respiratory } \\
\text { chain at haem } \\
\text { a3 division }\end{array}$ \\
\hline $\begin{array}{l}\text { BAPTA AM } \\
\text { 1,2-Bis(2-aminophenoxy)- } \\
\text { ethane-N,N,N',N'-tetraacetic } \\
\text { acid acetoxymethyl ester }\end{array}$ & $\begin{array}{l}32.5 \mathrm{mM} \text { in } \\
\mathrm{DMSO}\end{array}$ & $-18^{\circ} \mathrm{C}$ & $\begin{array}{l}100 \mu \mathrm{M} \\
(20 \quad \text { minutes } \\
\text { incubation at } \\
\left.37^{\circ} \mathrm{C}\right)\end{array}$ & $\begin{array}{ll}\text { Chelator } & \text { of } \\
\text { cytosolic } & \mathrm{Ca}^{2+} \\
\text { ions }\end{array}$ \\
\hline $\begin{array}{l}\text { Cyanide } \\
\mathrm{NaCN}\end{array}$ & $\begin{array}{l}1 \mathrm{M} \text { in } \mathrm{H}_{2} \mathrm{O} \\
\text { destillata }\end{array}$ & $\begin{array}{l}+4^{\circ} \mathrm{C} \\
\text { freshly } \\
\text { made }\end{array}$ & $\begin{array}{llll}100 \mu \mathrm{M} \text { or } 1 \\
\mathrm{mM} & & & \end{array}$ & $\begin{array}{l}\text { Inhibitor of } \\
\text { complex IV of } \\
\text { the respiratory } \\
\text { chain }\end{array}$ \\
\hline $\begin{array}{l}\text { Dantrolene } \\
\text { 1-([5-(4-nitrophenyl)-2-fu- } \\
\text { ryl]methyldeneamin)-imida- } \\
\text { zolidine-2,4-dione }\end{array}$ & $\begin{array}{l}20 \mathrm{mM} \text { in } \\
\text { DMSO }\end{array}$ & $+4^{\circ} \mathrm{C}$ & 20 or $40 \mu \mathrm{M}$ & $\begin{array}{l}\text { Ryanodine } \\
\text { receptor } \\
\text { blocker (ER) }\end{array}$ \\
\hline $\begin{array}{l}\text { FCCP }\left(\text { Tocris }^{\circledR}\right) \\
\text { Carbonyl cyanide-4-trifluoro- } \\
\text { methoxyphenylhydrazone }\end{array}$ & $\begin{array}{l}10 \mathrm{mM} \text { in } \\
\text { DMSO }\end{array}$ & $+4^{\circ} \mathrm{C}$ & $\begin{array}{llll}500 & \mathrm{nM} \text { or } 1 \\
\mu \mathrm{M} & & & \end{array}$ & $\begin{array}{l}\text { Protonophore } \\
\text { at the inner } \\
\text { mitochondrial } \\
\text { membrane - } \\
\text { "uncoupler" }\end{array}$ \\
\hline $\begin{array}{l}\text { Glutamate } \\
\mathrm{C}_{5} \mathrm{H}_{9} \mathrm{NO}_{4}\end{array}$ & $\begin{array}{l}1 \mathrm{M} \text { in } \mathrm{H}_{2} \mathrm{O} \\
\text { destillata }\end{array}$ & $-18^{\circ} \mathrm{C}$ & $\begin{array}{l}500 \mu \mathrm{M} \text { or } 1 \\
\mathrm{mM} \\
\text { (experiments) } \\
50 \mu \mathrm{M} \\
\text { (viability test) }\end{array}$ & $\begin{array}{l}\text { Agonist on } \\
\text { ionotropic and } \\
\text { metabotropic } \\
\text { glutamate } \\
\text { receptors }\end{array}$ \\
\hline
\end{tabular}




\subsection{Animal requirements and preparation}

Prior to animal preparation autoclaved glass cover-slips were laminated with Matrigel (BD Biosciences ${ }^{\circledR}$ ) and growth medium [medium (see 2.2.2.), $5 \%$ fetal bovine serum, $0.2 \mathrm{M}$ glutamine, B-27 supplement and antibiotics] and placed in a four-well plate $\left(\mathrm{Nunc}^{\circledR}\right)$. Matrigel and growth medium were mixed in a ratio of 1:50. $50 \mu \mathrm{l}$ of it were placed in the midst of each well. An incubation of one hour followed, during which animal preparation occurred under permanently ice-cold conditions.

Sprague-Dawley rats were used for preparation of primary hippocampal cell cultures. For reasons of cellular survival of the preparation and facilitated dissociation of single cells in the preparation process only neonatal pups no older than P5 (120 h after birth) were regarded eligible. Rapid decapitation ensured a painless death of the pup. The brain rapidly was extricated using the method shown below (Fig. 5) and placed in a Petri-dish containing Hanks' solution (HBSS) and $20 \%$ foetal bovine serum (FBS). The hemispheres were separated from each other removing both the cerebellum and the brain stem. Applying a scalpel and tweezers each hippocampus was carefully excised and cleaned from major blood vessels, membranes and fimbriae before being cut into smaller. During all these processes the ice-cold HBSS and FBS solution repeatedly was exchanged. Prior to digestion pieces were washed five times in HBSS/ 20\% FBS and subsequently HBSS. 

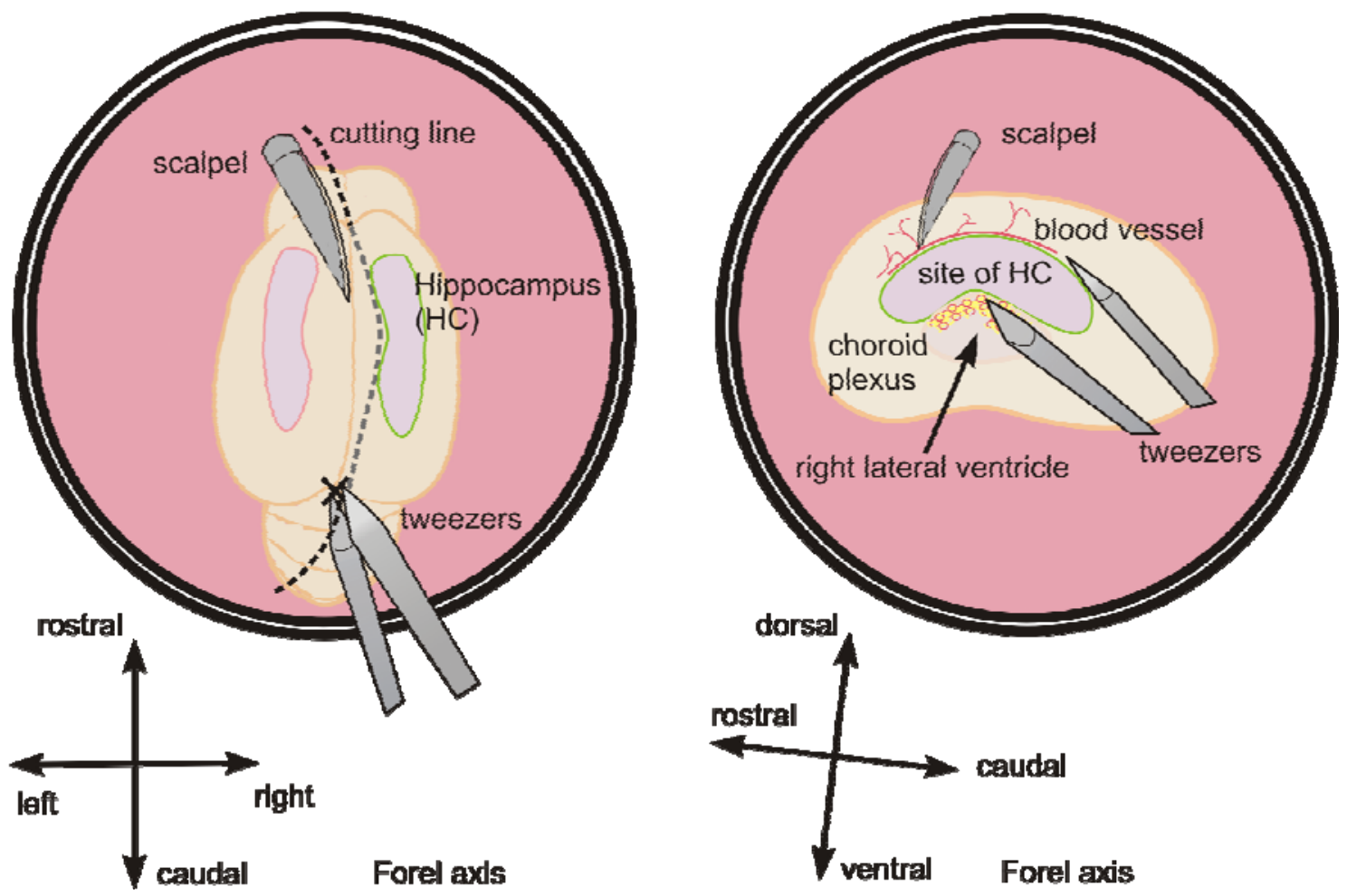

Figure 5 First steps of cell culture preparation

The brain is submerged in ice-cold HBSS and fixed with tweezers. Preparation takes place under a microscope. The right hemisphere is dissipated from the brain with a diagonal cut inserting between the hemispheres. Commissures can gently be severed with a drag of the scalpel. The separated hemisphere lies with the medial side on top. The hippocampus prominently protrudes if tweezers are placed between the choroid plexus and a remarkable blood vessel usually found dorsal of the hippocampus. The formation is gently loosened out of the hemisphere with the back of the scalpel and then carefully cleaned from meninges and other structures. The steps are repeated for the left hemisphere. All following steps are according to the main text.

The extracellular matrix of hippocampal pieces was digested in $2 \mathrm{ml}$ of digestion $\operatorname{mix}\left(137 \mathrm{mM} \mathrm{NaCl}, 5 \mathrm{mM} \mathrm{KCl}, 7 \mathrm{mM} \mathrm{Na}_{2} \mathrm{HPO}_{4}\right.$ and $25 \mathrm{mM}$ HEPES - buffered at $\mathrm{pH}$ 7.2) with an added $10 \mathrm{mg}$ of trypsin and $40 \mu \mathrm{l}$ of DNase during a ten-minute incubation at $37^{\circ} \mathrm{C}$. Washing in HBSS/ $20 \%$ FBS to stop the digestion followed again as described above. After three FBS washout cycles cell dissociation was achieved moving the pieces in a dissociation mix of $100 \mathrm{ml}$ HBSS containing $\mathrm{MgSO}_{4}$ to support membrane stability and again $40 \mu \mathrm{l}$ of DNase until distinct particles were no longer visible. A final washing step was executed using HBSS and FBS at $4^{\circ} \mathrm{C}$ and $1500 \mathrm{rpm}$ in a centrifuge for ten minutes. Supernatant was removed and the cellular sediment was re-suspended in $800 \mu \mathrm{l}$ of plating medium. 
Matrigel was removed from the cover-slips leaving a residual film of the adherent. $50 \mu \mathrm{l}$ of cell suspension were pipetted onto each cover-slip inside the four-well-plate. To allow cells to attach properly on the glass of the cover-slip wells the plates were incubated at $37^{\circ} \mathrm{C}$ for at least one hour. Subsequently, each well was covered in $600 \mu \mathrm{l}$ of isothermal plating medium.

The primary cultures received a total exchange of growth medium after $24 \mathrm{~h}$ and then after each $36 \mathrm{~h}$ in order to deliver new substrate and prevent the accumulation of toxic metabolic products.

To minimize metabolic and mechanical stress for the cell cultures they were transported quickly but carefully to the recording chamber inside a Petri-dish filled with warm ACSF and were allowed an accommodation time of 10 minutes prior to the first recording.

For the achievement of reliable results it was inevitable not to examine cells that either rested too short or too long in culture. Consideration of these aspects seemed helpful:

1. Glial cells just as neurones need to be given time to form new intercellular contacts as well as contact to the cover slip (Fischer and Kettenmann 1985). A minimum of two days in culture (DIC) was found adequate for this purpose. The general appearance in each well was verified by light microscopy prior to the staining process.

2. However, the longer glial cells were grown in culture, the less single cells were detectable. Significantly for astrocytes, they tend to form an almost homogenous cell-layer that prevents the recognition of cell boundaries.

3. Even under ideal storage conditions the viability of cells in culture will not excel much more than approximately two weeks. Furthermore, it remains unclear whether immature astrocytes dissociated from their original locus 
show a false or dedifferentiation over time. Thus an empirically estimated time range between $48 \mathrm{~h}$ to 7 days in culture was established.

\subsection{Cell labelling}

Various fluorophores have been used to monitor organelle structures or to obtain different dynamic measures of cellular and especially mitochondrial function. The following section explains use and properties of the probes in detail.

\subsubsection{JC-1}

JC-1 (MW $652 \mathrm{~g} / \mathrm{Mol}$; powder soluble in DMSO by Molecular Probes ${ }^{\circledR}$, USA) is a cationic probe which due to its marked change in fluorescence is particularly suitable to study the ratiometric dynamics of $\Delta \Psi_{\mathrm{m}}$. The lipophilic, and hence membrane-permeant, JC-1 monomers aggregate in polarized mitochondria due to their positive charge and emit at $590 \mathrm{~nm}$ (red). According to the manufacturers' description a peak excitation wavelength of $485 \pm 11 \mathrm{~nm}$ proved best to stimulate both aggregates and monomers. $485 \mathrm{~nm}$ were chosen for all JC-1 experiments in conventional wide-field microscopy. As mitochondria depolarize, e.g. during metabolic demand or in cell death, the J-aggregates dissociate again into monomers which emit a green fluorescence at a wavelength of $527 \mathrm{~nm}$. Already in 1991 Smiley et al. showed that the increase in red fluorescence follows linearly the increase in $\Delta \Psi_{m}-a$ fact that facilitates later ratiometric calculations of $\Delta \Psi_{\mathrm{m}}$ (Smiley et al. 1991).

JC-1 reacts relatively slowly to changes in $\Delta \Psi_{\mathrm{m}}$ compared to Rhodamine 123 (Rho123). However, JC-1 is the only optical probe allowing a true ratiometric analysis of $\Delta \Psi_{\mathrm{m}}$. Furthermore $\mathrm{JC}-1$ molecules seem to be less sensitive to changes in plasma membrane polarization than other $\Delta \Psi_{\mathrm{m}}$ sensitive dyes (Salvioli et al. 1997) and show reversible linear changes in ratiometric fluorescence values upon changes in $\Delta \Psi_{m}$ (Cossarizza et al. 1996). Similar to 
all cationic probes $\mathrm{JC}-1$ is taken up into the cell and each of its organelles according to its concentration following the Nernst equation. The aggregation leads to an excess of molecules inside the mitochondria. Other than Rho123 in a dequenching mode, JC-1 shows no reduction in fluorescence but an increase under this circumstance. The cytosolic green background fluorescence of the monomers diminishes.

A stock solution of $2 \mathrm{mg} \mathrm{JC}-1$ per $1 \mathrm{ml}$ DMSO was prepared and stored at $4^{\circ} \mathrm{C}$. $0.4 \mu \mathrm{l}$ of it were carefully added to the $800 \mu \mathrm{l}$ of medium of each well delivering a final dye concentration of $1 \mu \mathrm{g} / \mu \mathrm{l}$. Due to possible unwanted side effects of DMSO it was necessary to keep its concentration below $0.2 \%$ in the medium. In accordance with the manufacturers' advice the labelling was at $37^{\circ} \mathrm{C}$ for about 15 minutes.

\subsubsection{Rhodamine 123}

Rhodamine 123 (Rho123; MW $380.82 \mathrm{~g} / \mathrm{Mol}$ ) also allows visualization of relative changes in $\Delta \Psi_{\mathrm{m}}$ (Emaus et al. 1986, Duchen 1999, Foster et al. 2006). The mechanism is however totally different. Rho123 molecules are autofluorescent and at the applied concentration their fluorescence is substantially quenched in polarized mitochondria (dequenching mode). This means that Rho123 molecules form aggregates inside the organelle from a certain concentration on allowing accumulating more molecules than actually would be expected according to the Nernst equation (Lemasters and Ramshesh 2007, Pon 2007). Fluorescence is then decreased. During depolarization they leave the mitochondria and more molecules start emission at $529 \mathrm{~nm}$ - a process referred to as de-quenching. Unlike JC-1 there is only one emission wavelength. The peak excitation wavelength is $480 \mathrm{~nm}$.

Rho123 was dissolved in DMSO to produce a stock solution of $2.5 \mathrm{mg} / \mathrm{ml}$ that was stored at $4^{\circ} \mathrm{C}$. Cells were incubated at $37^{\circ} \mathrm{C}$ in a final concentration of 2.5 $\mu \mathrm{g} / \mathrm{ml}$ Rho123 for 25 minutes. 
Rho123 experiments were run in order to confirm findings of JC-1 experiments with a second potentiometric dye although a ratiometric analysis is not possible, since Rho123 is shows less distinct response to changes in $\Delta \Psi_{m}$ and is more prone to respond to cellular changes in potential as well as it shows a stronger bias due to quenching effects.

\subsubsection{Fluo-3 AM}

Fluo-3 AM (MW $1129.9 \mathrm{~g} / \mathrm{Mol}$; Molecular Probes ${ }^{\circledR}$, USA) is a non-ratiometric $\mathrm{Ca}^{2+}$ indicator used to analyze the dynamics of intracellular $\mathrm{Ca}^{2+}$ waves and microdomains in glia. A final dye concentration of $5 \mu \mathrm{M}$ was prepared out of a 2 $\mathrm{mM}$ stock solution from $50 \mu \mathrm{g}$ Fluo-3 AM powder dissolved in DMSO. Stocks were kept at $-18^{\circ} \mathrm{C}$. Despite of being a cell membrane permeant ester a more efficient penetration of the probe into the cell is achieved when the amphiphilic substance Pluronic ${ }^{\circledR}$ was added to the stock solution at a concentration of $10 \%$. Labelling took 35 minutes at $37^{\circ} \mathrm{C}$. Fluo-3 AM was excited at a wavelength of $485 \mathrm{~nm}$ and emitted maximally at $527 \mathrm{~nm}$.

\subsubsection{Further organelle labelling}

Mitotracker Red 580 FM and ER Tracker Blue-White DPX (both Molecular Probes $^{\circledR}$, OR, USA) were used for two-photon laser imaging to investigate the spatial connection of mitochondria and endoplasmic reticulum in astrocytes. Mitotracker Red $(724.0 \mathrm{~g} / \mathrm{Mol})$ was prepared as a $1 \mathrm{mM}$ stock solution in DMSO. The probe has an optimum excitation wavelength of $580 \mathrm{~nm}$ and has a peak emission at $644 \mathrm{~nm}$. The ER Tracker (MW $580.53 \mathrm{~g} / \mathrm{Mol}$ ) already arrives as a 1 $\mathrm{mM}$ stock solution. After a suggested excitation at $374 \mathrm{~nm}$ emitted fluorescence may vary from 430 to $640 \mathrm{~nm}$. It showed that labelling produced best results when applying the ER Tracker first at a final concentration of $1 \mu \mathrm{M}$ at $37^{\circ} \mathrm{C}$ for 20 minutes and consecutively applying the Mitotracker for $10-12$ minutes at a concentration of $500 \mathrm{nM}$ only.

DAPI Nucleic Acid Stain (Molecular Probes ${ }^{\circledR}$, OR, USA; $350.25 \mathrm{~g} / \mathrm{Mol}$ ) binds to 
double-stranded DNA. Its excitation wavelength is $358 \mathrm{~nm}$. The emission wavelength of $461 \mathrm{~nm}$ lets nuclei appear blue.

All three labelling dyes were excited at 790 to $800 \mathrm{~nm}$ of the two-photon laser. Since they are alkylates and toxic to living cells, instead of e.g. following the dynamics of mitochondrial transport only the visualization of subcellular structures was possible. All fluorescent probes were stored at $-18^{\circ} \mathrm{C}$.

\subsubsection{GFAP immunohistochemistry as an aid for cell identification}

The protocol demands a primary antibody to attach to the glial acidic fibrillary protein (GFAP) and a secondary antibody carrying the fluorophore that attaches to the primary antibody. Both protocol and antibodies were generously provided by Prof. Dr. G. Flügge (Dt. Primatenzentrum, Göttingen). GFAP is an important protein in the glial cytoskeleton and is not expressed in neurons. The primary antibody was polyclonal rabbit Anti-GFAP (Dako ${ }^{\circledR}$; code N1506) diluted in PBS to a 1:10 stock. The secondary antibody was labelled donkey Anti-rabbit IgG $\left(\right.$ Invitrogen ${ }^{\circledR}$ ) diluted to a $1: 2$ stock in glycerol. The fluorescent is Alexa $488^{\circledR}$ (excitation/emission peaks 495/519 nm).

A cover slip with a cell culture of a $72 \mathrm{~h}$ old pup (P3) was chosen at DIC 3. Growth medium was removed and the culture was gently rinsed with phosphate buffered saline (PBS). Then it was fixed in PBS/ 4\% paraformaldehyde (PFA) at room temperature for 20 minutes. Again it was carefully washed three times with PBS and then incubated at $37^{\circ} \mathrm{C}$ for 20 minutes in a PBS/ $0.2 \%$ Triton $\mathrm{X} / 10 \%$ FBS mix. Triton $X 100^{\circledR}$ (MW $625 \mathrm{~g} / \mathrm{Mol}$ ) is a detergent to permeabilize the cells for labelling. After a last rinse in PBS the slip was incubated at $7^{\circ} \mathrm{C}$ in a mix of PBS/ $0.2 \%$ Triton $\mathrm{X} / 1 \%$ FBS plus the primary antibody that was diluted by factor 200 to a final concentration of 1:2000. A day later the slip was rinsed with PBS and then incubated in the dark at room temperature for 1 hour. The secondary antibody was diluted to a final concentration of 1:700 using the same mix as for the primary antibody. Finally the cover slip was rinsed in PBS and 
eventually was dried at room temperature. A drop of mounting medium was applied on a glass slide and the cover-slip was most carefully placed on it. Microscopy started as soon as the mounting medium had dried granting tight adhesion of the cover-slip to the glass slide.

\subsection{Experimental set-up}

There were two different set-ups used for optical recordings - one set-up for wide-field microscopy and another for two-photon excitation laser imaging (TPELSM). Both of them are described in detail in the following section.

2.5.1. Set-up for both wide-field microscope and two-photon excitation laser imaging

To keep the cell culture vital during the recording a PVC submersion style chamber with a continuous flow of ACSF was used. The ACSF was kept in a warm water bath at $38^{\circ} \mathrm{C}$ and aerated with carbogen through a micro-porous stone. Separate gas pipes could be used for additional flasks containing drugs dissolved in ACSF. The ACSF ran in clean PVC hose. An air vessel was integrated to deliver a continuous flow and prevent bubbles and pressure oscillations in the submersion-style recording chamber and possible backflow to the ACSF bottle. A cassette pump (Watson Marlow 205S/CA ${ }^{\circledR}$ ) on roller bearings moved the ACSF forward at 35 revolutions per minute. This equals a flow velocity inside the hose of $1.6 \mathrm{~m} / \mathrm{min}$ - a delay which has to be considered in drug application. The ACSF cooled down on its way to the recording chamber and was repetitively measured to have 33 to $34^{\circ} \mathrm{C}$ which is an adequate temperature for hippocampal cell cultures. The ACSF, and especially ACSF of altered composition or containing dissolved drugs, was discharged from the recording chamber into a hypobaric flask. 


\subsubsection{Optical microscope wide-field imaging}

The optical imaging set-up for conventional wide-field microscopy contained two light sources (Fig. 6a). A source for white light inside a gooseneck lamp was used for transmission pictures or for visual focussing of the cell culture prior to recording. Alternatively, a polychromatic xenon gas $150 \mathrm{~W}$ high stability light source (Ushio Inc. ${ }^{\circledR}$, Japan) with a bandwidth of $\pm 15 \mathrm{~nm}$ was applied. The wavelength could precisely be adjusted to the specific fluorophore applying a monochromator (Polychrome V by Till Photonics ${ }^{\circledR}$, Gräfelfing, Germany) via scanner mounted diffraction grating. The light source was fibre-coupled to the microscope. The choice could be changed and saved within the Till Vision ${ }^{\circledR}$ (see 2.6.1.) protocols.

All light bulbs and particularly new ones were preheated for 20 minutes prior to usage to grant a stable monochromatic light emission. None of the bulbs was used longer than 500 hours in total according to the producers' advice.

For JC-1 recordings at $485 \mathrm{~nm}$ emission wavelength a $505 \mathrm{~nm}$ dichroic mirror DCLP was applied (Fig. 6b).

The upright microscope (Axiotech Vario ${ }^{\circledR}$, Zeiss, Oberkochen, Germany) was equipped with a 63x, 1.0 NA water immersion objective (Apochromat ${ }^{\circledR}$, Zeiss) for Fluo-3 AM recordings or a 100x, 1.0 NA water immersion objective (Achroplan ${ }^{\circledR}$, Zeiss) for JC-1 recordings. The cell culture was slightly submerged during the entire recording. The camera was a highly-sensitive CCD camera (Imago $\mathrm{QE}^{\circledR}, \mathrm{PCO}$ Imaging, Kelheim, Germany) that was either directly attached to the microscope or to the Dual-View ${ }^{\circledR}$ device for JC-1 recordings. 


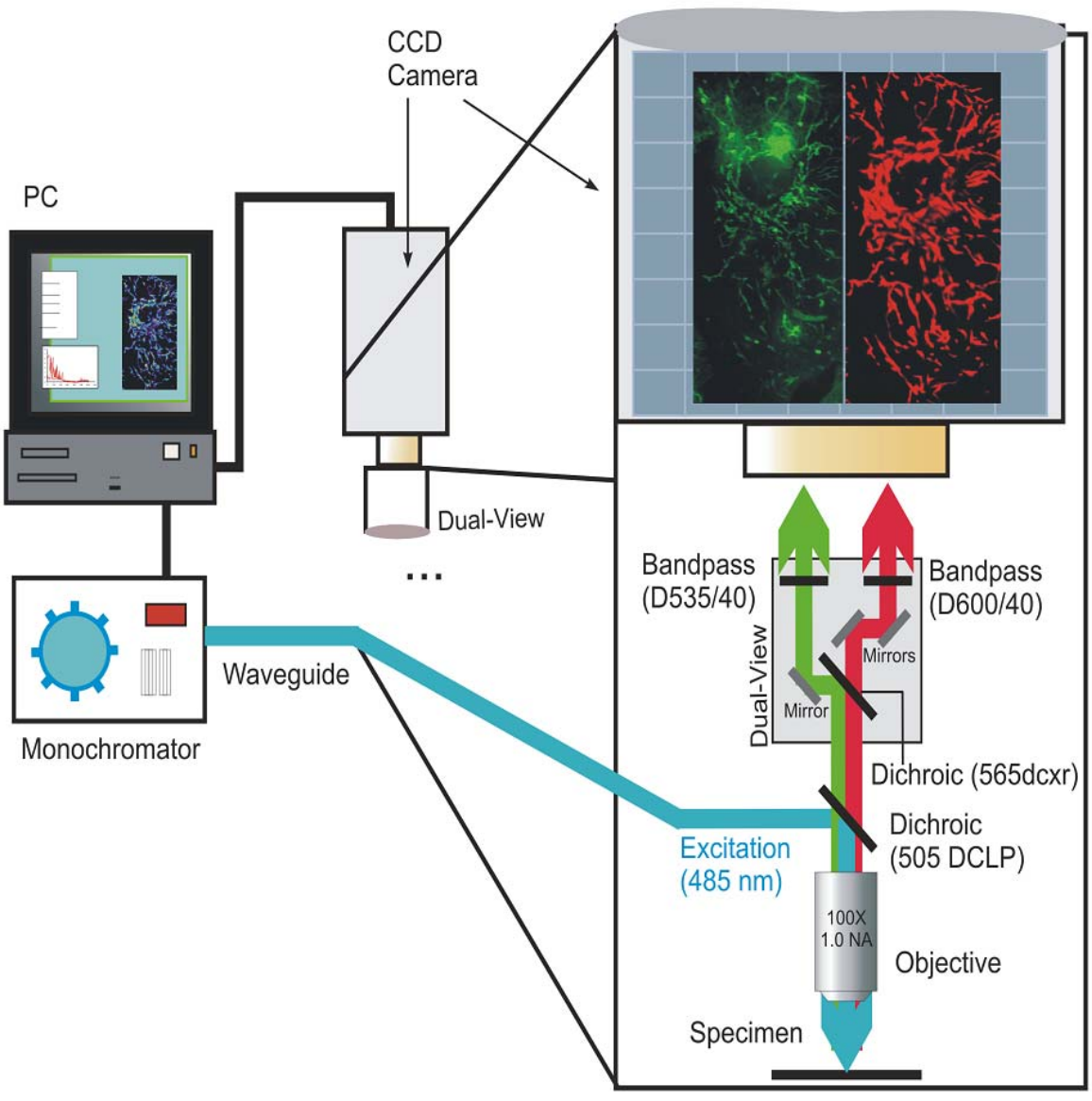

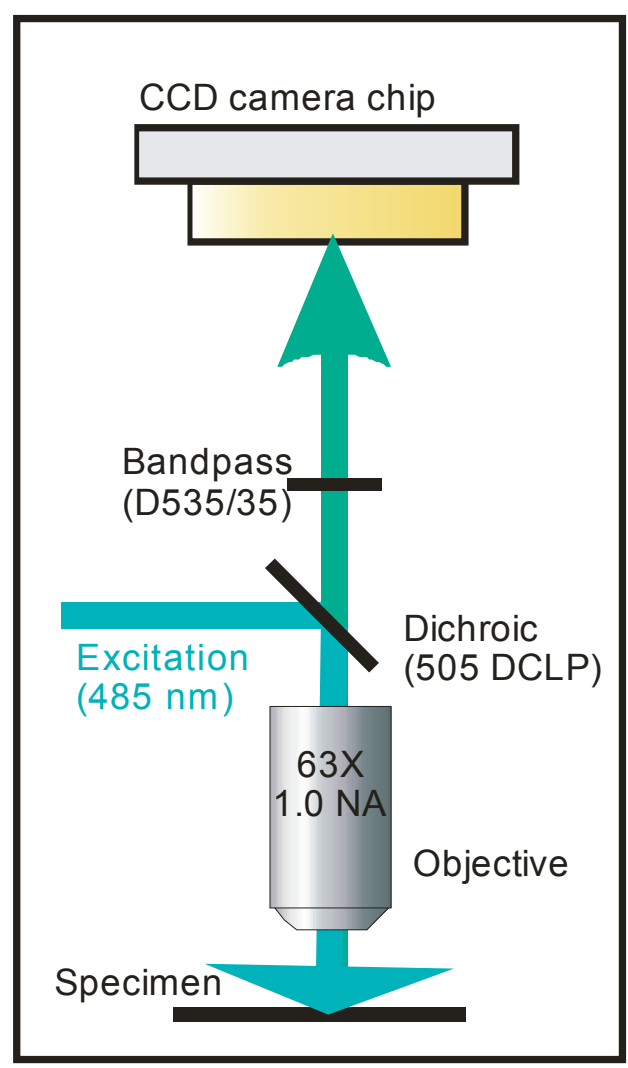

Figure 6a Wide-field imaging set-up

For JC-1 recordings $120 \mathrm{~ms}$ pulses of $485 \mathrm{~nm}$ light from a monochromatic light source enter the microscope via a waveguide. A 505DCLP dichroic reflects the excitatory light to the specimen. The emitted light of two different wavelengths can pass the dichroic and enters the DualView device. Here a second dichroic (565dcxr) splits the red and green images according to wavelength. The emission is filtered for the respective wavelength applying bandpass filters. Mirrors can be changed in angle to produce the greatest parallelization possible of both images on the CCD chip of the camera. The image is sent back to the $\mathrm{PC}$ whose Till Vision programme runs the recording. The specimen is magnified by a $100 \mathrm{X} 1.0 \mathrm{NA}$ objective. A C-mount between Dual-View and camera was neutral to magnification.

Figure 6b Adaptation for Fluo-3 AM fluorescence

For Fluo-3 AM images a larger overview on several astrocytes was necessary. Therefore a $63 \mathrm{X} 1.0 \mathrm{NA}$ objective was applied. The excitation wavelength was 485 $\mathrm{nm}$. The 505 DCLP dichroic proved functional. The emitted fluorescence was registered directly by the CCD camera after having applied a D335/35 nm bandpass filter.

(Both images were modified from my publication in submission.) 
Dual-View $^{\circledR}$ (Optical Insights ${ }^{\circledR}$, Tucson, USA) is an optical image splitter device that can be inserted into the emission light path between the microscope and the camera (Fig. 6a). Its filter set comprises a $565 \mathrm{dxcr}$ beam splitter to spectrally separate the red and green emissions of e.g. JC-1 labelled mitochondria and two bandpass emission filters of D535/40 (green emission) and D600/40 (red emission; both Chroma Technology Corporation ${ }^{\circledR}$ ). The spectrally separated half images appear simultaneously on the CCD chip of the camera allowing the observation of changes in $\Delta \Psi_{\mathrm{m}}$ in real time. It is therefore ideal for JC-1 recordings with the intention of ratiometric analyses.

To obtain ideal results the angle of all mirrors inside the device had to be carefully adjusted to produce correctly aligned images of the observed cell culture region on the CCD chip on the $x$ and $y$ axis. Without complementary images neither overlay images nor ratio images are possible to produce. However, since correspondence of mirrors could only be achieved manually slight errors could secondarily be eliminated within the MetaMorph programme. For Fluo-3 AM and Rho123 labelled cells, which only emit at one wavelength, the image splitter was not applied and instead the 505nm DCLP dichroic mirror and a D535/35 $\mathrm{nm}$ bandpass emission filter were used only.

Wide-field microscopy with monochromatic light has several drawbacks such as exposure of the specimen and fluorophore to a UV light component causing toxic side effects or also a poor axial resolution which impairs a realistic impression in a three-dimensional perspective, since all light emission is a super-imposed cumulation of all fluorescence at one spot.

\subsubsection{Two-photon excitation laser scanning microscopy}

The concept of two-photon excitation laser scanning microscopes (TPELSM) was presented to the scientific community only in 1990 (Denk et al. 1990). The idea allows depicting living cells three-dimensionally, as so-called z-stacks, at the nanometre range. The fluorophore will only be excited if two photons of 
about the double wavelength to the normal excitation wavelength are directed to the same space by the scanning mirrors to be absorbed both at the same time (Fig. 7) (Göppert-Mayer 1931). This means that different from wide-field microscopy TPELSM produces images that only depict structures on the scanned level and not summarizations of all fluorescence found on all axially super-imposed structures.

\section{Photon 2 Photons}

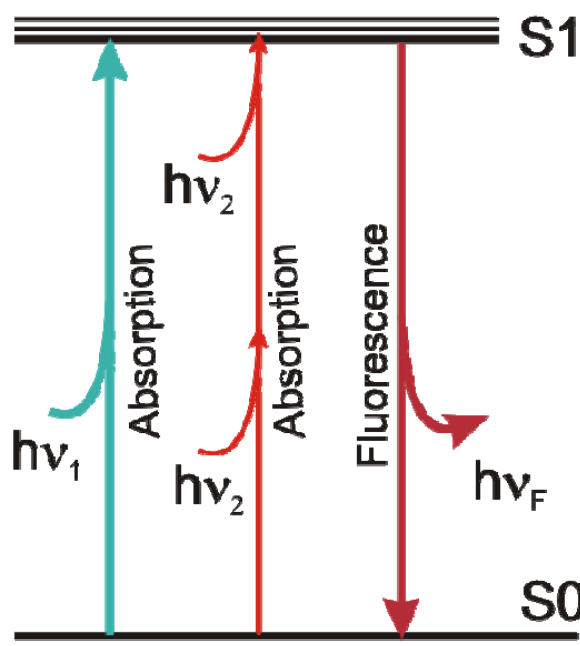

Figure 7 The Jablonski diagram

This diagram visualizes the physical theory behind TPELSM. In fluorescence microscopy the fluorophore absorbs a defined quantum of energy ( $h v_{1}$; $h$ : Planck's constant; $v$ : frequency). The molecule is then excited to a higher energetic level (S1). This takes about $10^{-15}$ seconds. If two photons of double this wavelength (and hence half the energy; $h v_{2}=h v_{1} / 2$ ) excite the fluorophore during this short period, it may also reach $\mathrm{S} 1$. As the fluorophore turns to the ground state S0 a photon is emitted - the fluorescence seen. For energetic reasons this emission will always have a longer wavelength than the excitation.

To achieve this temporal coincidence of simultaneous photon absorption, that according to physical deliberations does not allow more than 100 attoseconds, a pulsed laser beam is generated by a Titan:Sapphire laser (Tsunami ${ }^{\circledR}$, Model 3941-M3BB) delivering ultra-fast 100 femtosecond pulses at $80 \mathrm{MHz}$ (Fig. 8). The applied fluorophores could all be sufficiently excited by light of $800 \mathrm{~nm}$. The original laser beam of $20 \mathrm{~W}$ diode laser bars pumps an all-solid-state laser (Millenia Vs ${ }^{\circledR}$, Spectra Physics ${ }^{\circledR}$ ) at $5 \mathrm{~W}$ into the Tsunami ${ }^{\circledR}$ resonator cavity and leaves it at less than $2 \mathrm{~mm}$ of diameter. The beam runs in a safety tube until being expanded by a set of lenses prior to entering the scanning element of two $4 \mathrm{~mm} \mathrm{x}$ - and $\mathrm{y}$-mirrors, the galvanometer driven VM500C scanners and MiniSAX control units (all General Scanning Inc. ${ }^{\circledR}$ ). The beam then enters the upright microscope (Olympus ${ }^{\circledR}, \mathrm{BX} 51 \mathrm{WI}$ ) via the scan lens, the tube lens and the objective (IR-optimized 20x 0.95 NA; XLUMPlanFL, Olympus ${ }^{\circledR}$ ). The working distance is $2 \mathrm{~mm}$. Furthermore the specimen placed in a submersion style chamber can be viewed through the oculars via a white light source. 


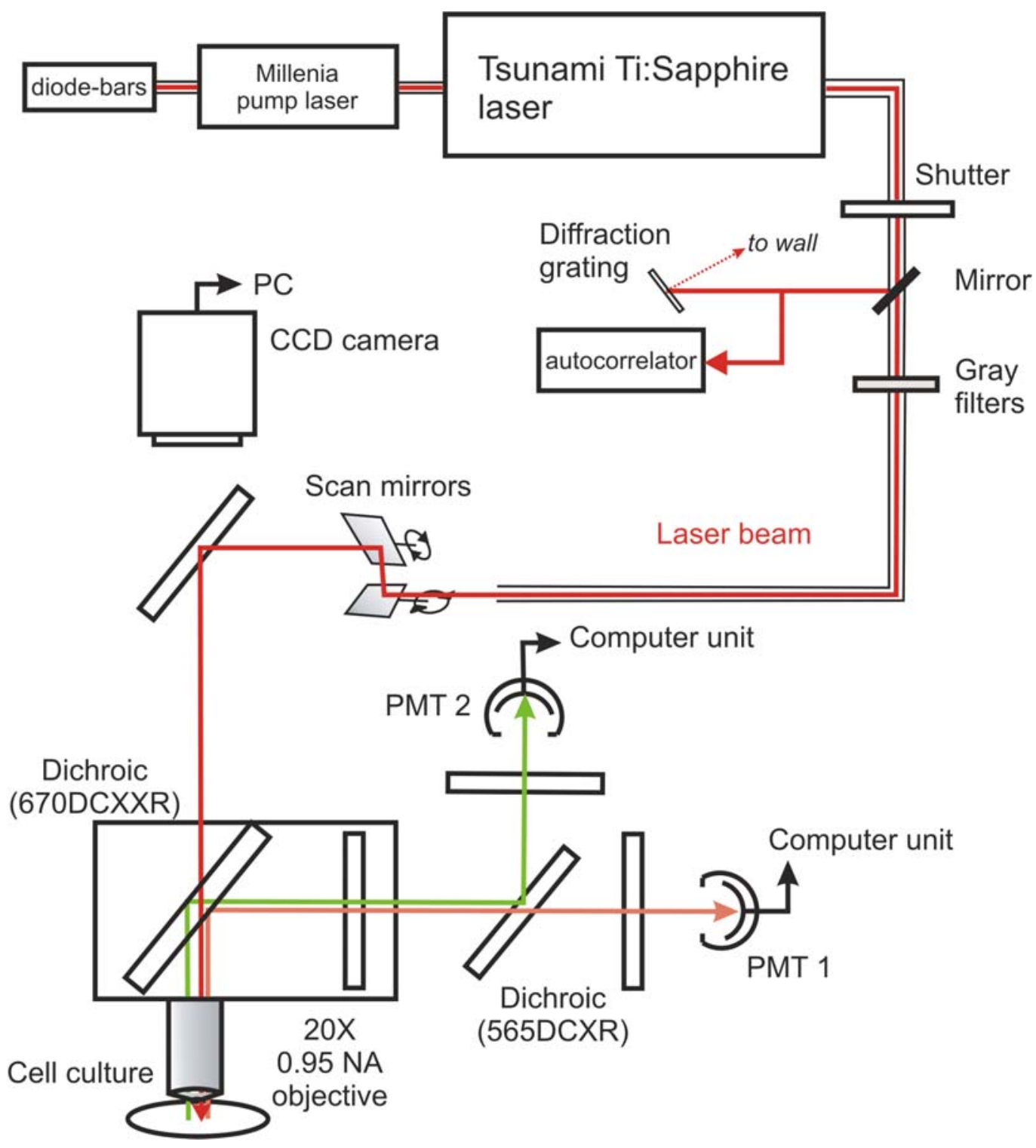

Figure 8 The two-photon excitation laser scanning microscope (TPELSM)

This simplified scheme particularly omits mirrors and lenses. The laser source are $20 \mathrm{~W}$ laser diode bars that are pumped by the Millenia all-solid state laser to the Tsunami Titan-Sapphire laser source that can provide laser beams at different wavelength. A high-frequency pulsed laser beam of $<2 \mathrm{~mm}$ diameter at 5 W leaves the Tsunami. To check for a correct pulse and wavelength the first order of the beam is reflected to a nm-scale on the wall making use of the fact that light is scattered at different angles depending on the wavelength. Further mirrors and filters shape the beam prior to entry into the photoresist foil covered microscope unit. The near infrared beam is directed by the scanning mirrors passes a 670DCXR dichroic and enters the upright microscope. Emitted light on the other hand is reflected and filtered before being split by a second 565DCXR dichroic according to wavelength. Emissions of the green and red spectrum are separately registered by two photon multipliers (PMT). Sensitivity of the PMTs can be adjusted manually. The 20X 0.95 NA objective can be focussed on the z-axis by a Pifoc unit (not shown). A white light source is fixed under the cell culture chamber for transmission images (not shown). (modified from (Müller et al. 2003) p. 1749).

During recording the axial focus can precisely be controlled using a piezo-driven objective positioning system (Pifoc P-721 ${ }^{\circledR}$, Physik Instrumente ${ }^{\circledR}$ ). The emitted 
light is measured by two wide-field-detection photomultipliers (H7421-40, Hamamatsu $^{\circledR}$ ) for red and green wavelength in JC-1 experiments and was intensity coded in TTL pulses. A digital signal processor (Adwin-Gold-ENET ${ }^{\circledR}$, Jäger $\mathrm{GmbH}^{\circledR}$ ) controls both scanning and data acquisition. Scanning time, light exposure time, repeat of scanning, distance of focus levels for z-stacks and digital zoom could all be selected by a linux-based control software programme. Equally, the assembled images could be depicted and saved for further processing, e. g. in MetaMorph ${ }^{\circledR}$. Usually a test scan was run followed by a series of five scans at 2000 steps per scanning line at the largest zoom possible. Longer scanning times mean better signal-to-noise ratio but also greater possible photo damage to the structure in focus.

The entire experimental set-up as well as the computer programmes applied are custom-built and greatly were created by members of the Müller lab (Müller et al. 2003). For non-laser white light transmission images an additional digital CCD camera (Micromax ${ }^{\circledR}$; Princeton Instruments ${ }^{\circledR}$ ) was installed.

TPELSM has several advantages over one-photon confocal laser scanning microscopy. The double wavelength of each of the photons needed for excitation is close to near infrared which on the one hand reduces phototoxic damage to both the living specimen and the fluorescent probe and on the other hand means that the beam is less scattered. The latter is advantageous for deeper penetration into tissue, e. g. to produce z-stacks, and for focussing. Regions outside this tiny space, in which simultaneous absorption of two photons occurs, are spared. Furthermore the focussed excitation produces only emission inside the focus which makes the use of a pinhole as in confocal microscopy superfluous. However, although contrast is better, which describes the apparent resolution, TPESLM is not superior to one-photon excitation as regards to factual resolution (Sheppard 1990). A limited drawback, that needs to be mentioned, is that TPELSM usually demands higher rating power of the laser to produce the same intensity of fluorescence, since the excitation depends on the probability of photons to be in the same space at the same time, which itself 
depends on the photon intensity. Still TPELSM proved superiority particularly against conventional wide-field microscopy concerning greater resolution and the possibility to acquire a three-dimensional, realistic view of subcellular structures.

\subsection{Image acquisition and processing}

All acquired images were processed with the appropriate set of software to perform a more refined and complex analysis allowing better resolution and statistical calculations of the data.

\subsubsection{Till Vision ${ }^{\circledR}$}

Till Vision $^{\circledR}$ (Till Photonics ${ }^{\circledR}$, Gräfelfing, Germany) is a software designed for scientific imaging and image processing which can save individually created protocols. Protocols contain wavelength and exposure time of the monochromatic excitation light for fluorescence imaging. Single images may be taken as a reference for continuous sequences. The frequency of camera activation (frame rate) as well as the total number of images taken are also part of the protocol. One may define automatic pixel binning of the image. In a $2 \times 2$ binning protocol the image data of four adjacent pixels, two pixel coordinates on the $\mathrm{x}$ axis and two on the $\mathrm{y}$ axis respectively, are averaged producing a resulting image of half the pixel count of the original on the CCD chip. This allows an increased contrast and at the same times a decreased resolution. The advantage is a reduction in required storage capacity. Within the image regions of interest (ROI) may be defined and saved in a reference image, whose changes in intensity on a priorily chosen colour range can be followed on a real time graph during the course of sequential imaging.

At the end of the imaging sequence the colour range may be adjusted if needed. The single images can be run as a film sequence of variable speed to give a 
first analysis of the image dynamics and finally this sequence can be exported from the original workplace to other folders and formats for offline image processing such as done by MetaMorph ${ }^{\circledR}$.

\subsubsection{MetaMorph Offline 7.1. ${ }^{\circledR}$}

MetaMorph Offline ${ }^{\circledR}$ (Universal Imaging Corporation/Molecular Devices ${ }^{\circledR}$ ) is an image processing software. Single exported images of Till Vision ${ }^{\circledR}$ or TPELSM recordings can be reassembled to a film sequence ("stack") as the basis for further analysis. MetaMorph ${ }^{\circledR}$ allows individual colour scaling of images in several bit ranges as well as visualization in different colours including a pseudo colour mode that was particularly helpful in discerning the polarization of mitochondria. The programme can correct the movement of cells during the recording applying an alignment function for consecutive images or the corresponding images of the two different red and green channels. The exact alignment of two images is of utter importance for overlay and ratio images in Dual-View ${ }^{\circledR}$ recordings as well as all analyses of light intensity over time. Other than conventional wide-field images TPELSM images of JC-1 labelled cells did not need alignment as long as they were acquired simultaneously because of their two different photomultipliers that delivered the exact same scanning locus rather than the single CCD chip used for Dual-View ${ }^{\circledR}$ based microscopy.

MetaMorph ${ }^{\circledR}$ can show statistical aspects of individually placed regions or lines of interest. The function can describe light intensity, and hence polarization, over time, mitochondrial length and polarization. This means that the programme is ideally suited for morphometric and spatiotemporal analyses. The data can be exported as a text file to Microsoft Excel ${ }^{\circledR}$. The exact location of $\mathrm{ROI}$ was saved with a screenshot.

Furthermore MetaMorph ${ }^{\circledR}$ was used to carefully ameliorate the resolution of individual mitochondria with a filter. This was especially necessary for all analyses, which required the colour threshold function such as analyses of 
length and polarization. The threshold function can define a region of interest.

For ratio and overlay images of Dual-View ${ }^{\circledR}$ images MetaMorph ${ }^{\circledR}$ provided a "split view" function, which allowed the separation of the two channels of the image into two paired images that possibly needed a secondary alignment prior to proceeding. In TPELSM recordings this step could be omitted. Arithmetic processing functions produced the overlay or ratio image.

\subsubsection{Microsoft Excel ${ }^{\circledR}$}

All statistical and quantitative analyses of $\mathrm{ROI}$ or other defined structures chosen by MetaMorph ${ }^{\circledR}$ region tools were graphically plotted applying Microsoft Excel $^{\circledR}$. MetaMorph ${ }^{\circledR}$ region analysis tables had undergone a preceding step of export into a text file.

The variables were plotted either against time in seconds. Changes of $\Delta \Psi_{\mathrm{m}}$ were hardly comparable as long as absolute colour values of the regions were regarded. Therefore values were normalized depicting only relative changes against a pre-treatment baseline whose value was defined as $1(=100 \%)$. Since blinking mitochondria usually turn into a state of higher polarization, which is represented as a peak in the trace, their baseline polarization value of brightness was chosen. If possible the average of the first five values was found eligible to mark this reference value defined as 1 in a normalized plot.

Most data is depicted as a line or scatter plot. The Excel average and standard deviation functions were used for statistical calculations.

\subsection{Statistical Analysis}

The data obtained for this dissertation were obtained from the cultured hippocampal cells of 28 rat pups. All experiments were at least run on the cells 
of three different pups to minimize the possibility of false results due to individual differences of the animal or the preparation. 210 recordings were made with JC-1 labelled cells and 56 recordings applying Fluo-3 AM. TPELSM recordings were made with cells cultures from 8 rat pups.

All data analyses are given as mean \pm standard deviation. Data were found significant at a 95\% confidence level (two standard deviations). Correlation coefficients were found high between 0.5 and 1or -0.5 and -1 respectively. 


\section{Results}

Astroglia are ideal cells for studies on a subcellular level due to their flat and spread out growth in cell cultures. Particularly mitochondria are not as densely packed as for example in neurones, which makes optical resolution of a single mitochondrion feasible without being forced to disrupt the functional organelle network in order to produce isolated mitochondria. Fluorescent dyes for morphological and functional imaging of astrocytes were bulk loaded and visualized with high-resolution microscopy. Short exposure times and the focussed laser beam applied in TPELSM largely spared the functionality of the cultured cells during the recording process.

In summary, these were the prerequisites to make an analysis of mitochondrial structure, distribution and polarization under physiological and pathological conditions in astroglia possible.

\subsection{Choice of astrocytes}

A specimen of a representative cell culture (age of pup 3 days; 3 days in culture) was immunolabelled against GFAP to validate the morphological choice of the analyzed astrocytes. GFAP is highly selective, for it is not present in neurones. TPELSM and conventional wide-field microscopic images of cells immunolabelled against GFAP revealed an astoundingly large morphological variety of glial cells within the cell culture (Fig. 9). Since I had focused exclusively on those cells, which - due to their flat shape - could classically be described as astrocytes, it was surprising to realize how many more shapes exist. However, since anti-GFAP-labelling is not a marker for astrocytes but for all glial cells including oligodendrocytes and some microglia subspecies, it was probably a wise choice not to have chosen cells of different shape in order to grant a most purely selected population of astrocytes as study objects. 


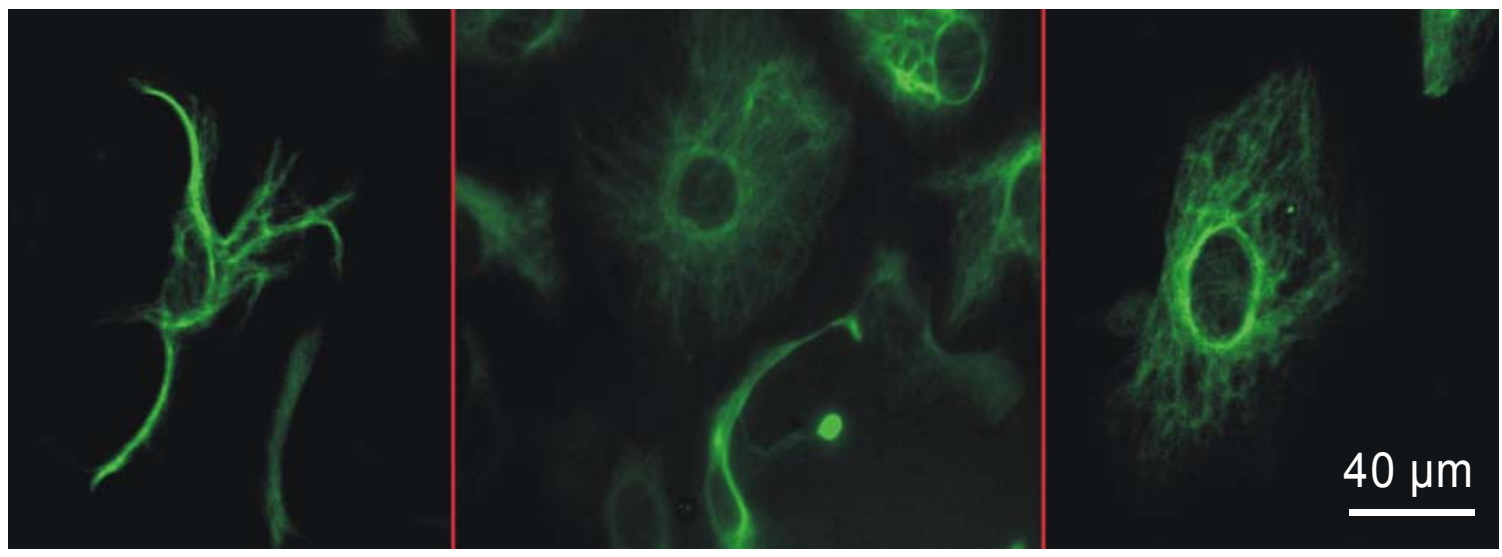

Figure 9 Overview of GFAP immunolabelled rat hippocampal astrocytes. Note the great variety of possible shapes of various glial cells. For all later experiments only those of roundish, widely spread shape were recorded (right image).

\subsection{Morphological variety and flexibility of mitochondria}

Research increasingly corrects the misconception of mitochondrial uniformity on the subcellular level. For mitochondria a great variety in shape and localization has already been published. I focused on eliciting heterogeneity of mitochondrial morphology, and thus assumingly function, in vital astrocytes - a cell type whose diverse functions are similarly now discovered.

In all conventional wide-field experiments performed on either JC-1 or Rho123 labelled cells the recording started with a perfusion of ordinary ACSF for at least two minutes. The most brilliant images were analyzed in this early phase according to mitochondrial morphology and cytoplasmic distribution. The effect is that a large set of recorded astrocytic mitochondria could be assessed at the same time of recording averting a bias deriving from phototoxic or pharmacologic damage to mitochondria and the astrocyte.

The protocol for shape and distribution analyses is given here:

1. 23 ratiometric Dual-View ${ }^{\circledR}$ and 5 TPELSM images were adjusted to an 8bit scale of 256 colour levels in MetaMorph ${ }^{\circledR}$ and optimized for mitochondrial contrast against the cytosol. To obtain ratiometric images 
intensity values of the red JC-1 channel colour values were multiplied by 5 and then divided by the green channel (Ratio $=\mathrm{JC}-1_{\text {red }}{ }^{*} 5 / \mathrm{JC}-1_{\text {green }}$ ). The coefficient 5 was introduced in order obtain ratio values from 0 (highly depolarized) to 255 (highly polarized) by analogy with the 8-bit colour scale chosen.

2. A threshold function was applied to separate the background furthermore from the fluorescent mitochondria.

3. An open-close morphology filter was applied to erase accidentally bright single pixels that would be a bias in the following integrated morphometric analysis.

4. The analysis was performed selecting individual mitochondria appearing inside the threshold as regions of interest by mouse-click, to avoid large mitochondrial clusters from being included.

5. Parameters analyzed for each mitochondrion were length and pixel coordinates of the centre of the structure as well as its area. For each astrocyte the highest possible number of individual mitochondria was chosen.

\subsubsection{Differences in mitochondrial shape}

Mitochondria showed a great heterogeneity in length. Granular shapes of $<1 \mu \mathrm{m}$ in length were found as well as particularly long mitochondria of up to about 21 $\mu \mathrm{m}$ (Fig. 10). These long and less densely packed mitochondria appeared to be preferably in the periphery of the astrocyte.

The morphological analysis approach mentioned in 3.2. was applied to 33 astrocytes - 2343 mitochondria were analyzed. The bar diagram (Fig. 11) describes the distribution of length in these mitochondria. As can be taken from 
the diagram all possible lengths from 0.5 to $21.5 \mu \mathrm{m}$ are present. $10.4 \%$ of mitochondria had a granular appearance $(<1 \mu \mathrm{m})$ and $11.5 \%$ were $5 \mu \mathrm{m}$ or longer, which means they belong to a set of long mitochondria. The majority of mitochondria $(78.1 \%)$ measured between 1 and $<5 \mu \mathrm{m}$. The average mitochondrial length was $2.48 \pm 2.33 \mu \mathrm{m}$.

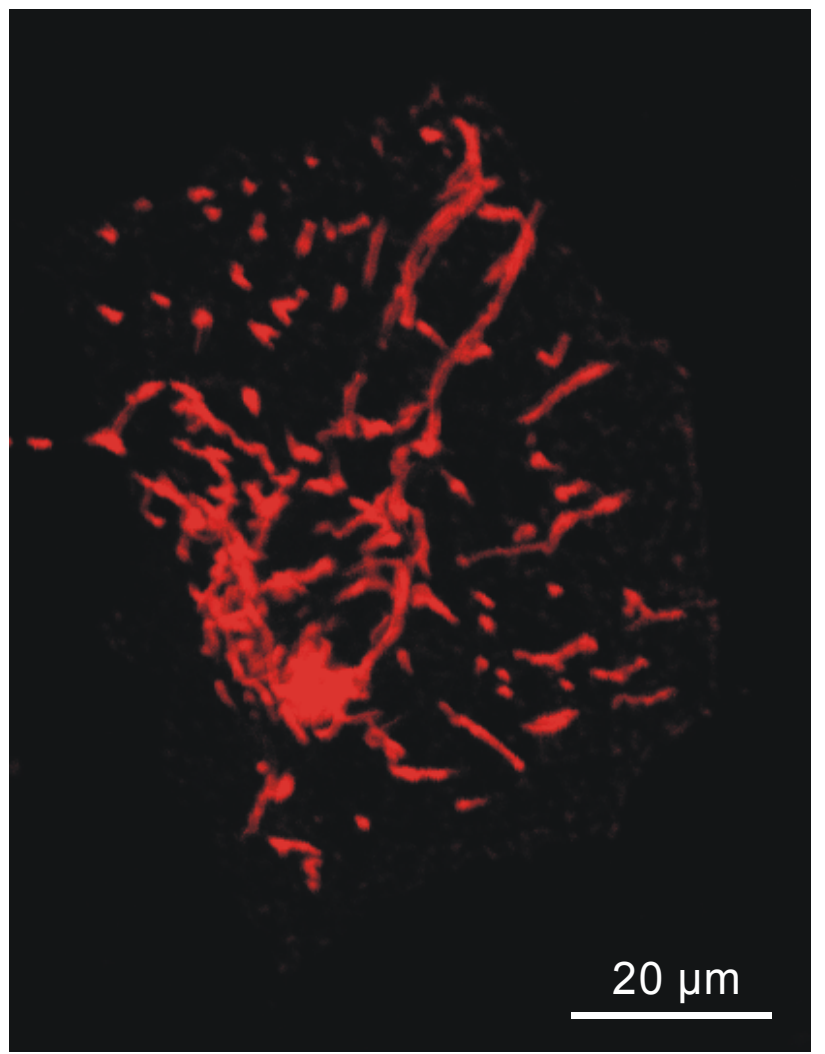

\section{Figure 10}

A TPELSM overview showing an astrocyte with mitochondria of variable length

\section{Figure 11}

Frequency distribution of mitochondrial length of 2343 randomly chosen mitochondria

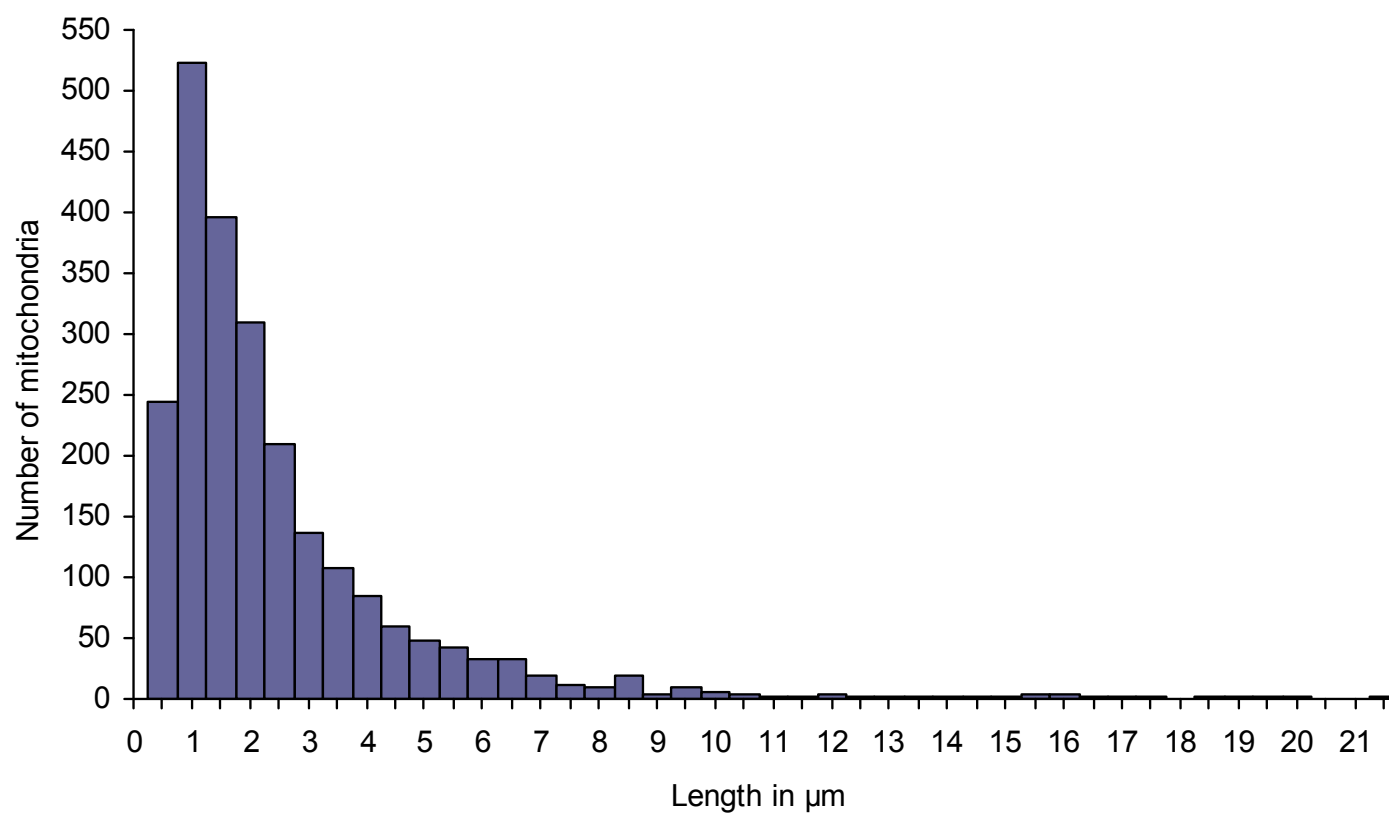


Since mitochondria sometimes were not only straight in shape, but also curled and possibly not clearly distinguishable from each other by a threshold function as they, particularly in the perinuclear region, were organized in densely packed clusters, a confirming second analysis on 917 mitochondria of 16 astrocytes was performed. The length of mitochondria could be measured in pixel values applying a line region tool in MetaMorph ${ }^{\circledR}$ and then be converted to their actual length in $\mu \mathrm{m}$ using this formula:

$$
1 \mathrm{px}=\frac{\text { (pixel size in } \mu \mathrm{m} \text { on the } \mathrm{CCD} \text { chip })^{*}(\text { binning factor })}{(\text { magnification factor })^{*}(\mathrm{C}-\text { Mount factor })}
$$

The usual conversion was $1 \mathrm{px}=0.129 \mu \mathrm{m}$ after having inserted $6.45 \mu \mathrm{m}$ as pixel size in $\mu \mathrm{m}$ on the CCD chip, a binning factor of 2, a 100x objective and a neutral C-mount magnification factor of 1 . This formula was used for all conversions from pixel to $\mu \mathrm{m}$.

For this method the mitochondria had to be selected manually. However, it allowed considering values of mitochondria that otherwise would not have been considered applying a decent and largely automated threshold function in MetaMorph $^{\circledR}$. The bar diagram (Fig. 12) greatly confirms the results based on threshold selected mitochondria. $6 \%$ of mitochondria can be considered granular. $11.6 \%$ are $5 \mu \mathrm{m}$ or longer. The rest lies in between. The average mitochondrion measures $2.58 \pm 1.70 \mu \mathrm{m}$ in length.

This analysis interprets morphological findings at the beginning of a recording. Analyses of mitochondrial movement and change of shape are described below. 


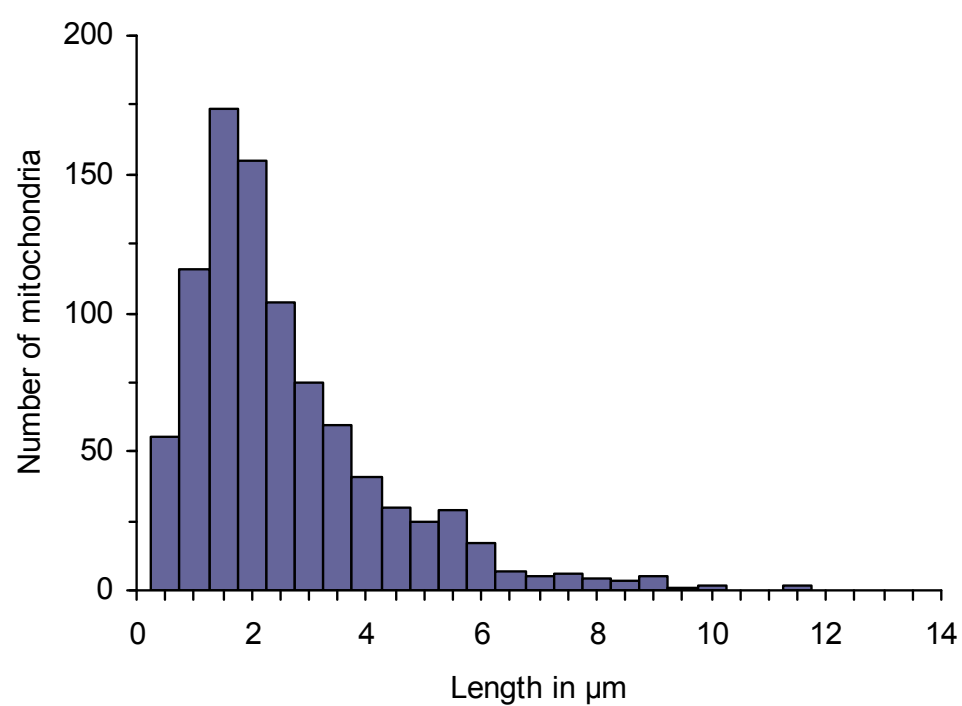

Figure 12

Secondary analysis of a frequency distribution of mitochondrial length - Most mitochondria are not much longer than 2 or $3 \mu \mathrm{m}$. There are exceptions however.

\subsubsection{The subcellular distribution of mitochondria in astroglia}

The distribution of mitochondria was examined for two different criteria: 1.) the total density of mitochondria in comparison to the entire cytosol and 2.) local cytosolic differences in mitochondrial density.

In order to give an analysis of the density of mitochondrial packing in the cytosol ratiometric images of $20 \mathrm{JC}-1$ labelled astrocytes were thresholded, binarized and filtered as described in 3.2.. This time all mitochondrial structures within the astrocyte were included for area measurements regardless of a possible resolution to the single mitochondrial particle. The values of all areas were added. In a second step the area of the astrocyte was calculated and the area of the nucleus subtracted. The area of all mitochondria was then divided by a total area of the astrocyte.

Mitochondrial structures occupied a share of 15.0 to $50.6 \%$ of the entire cytosol in focus. The average value was $33.2 \pm 8.9 \%$.

It is important to stress that this method can only estimate a mitochondrial density on a two-dimensional level since the image does not depict the three- 
dimensional state. However, since glial cells are very flat extrapolations can be made.

The density of mitochondria varied largely among the individual astrocytes. In a quantitative analysis of 114 astrocytes applying a threshold function to obtain a better contrast of mitochondrial structures against the cytosolic background $77 \%$ of all astrocytes (88 out of 114 ) showed the highest mitochondrial density in the perinuclear area.

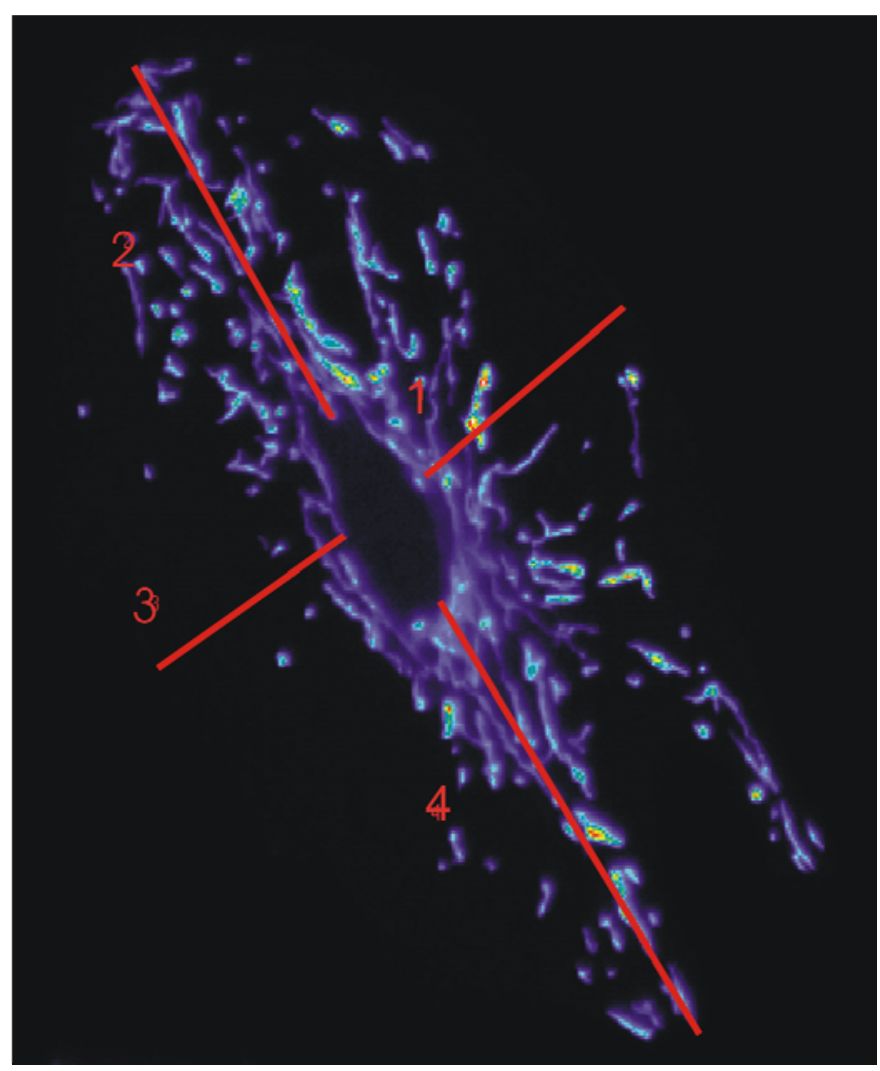

\section{Figure 13}

Intensity profile of light particles (mitochondria) and dark background (cytosol) as a schematic overview of a linescan - The thin red lines mark the lines of interest studied for average brightness to determine the density distribution of the mitochondria.

For another analysis on 16 randomly chosen astrocytes 39 lines of interest of at least $20 \mu \mathrm{m}$ across the cytoplasm analyzed starting from the edge of the nucleus (Fig. 13). The average intensity measured was transformed into a normalized averaged density profile. First, the intensity values on an 8-bit scale of all pixels on the line scan were measured. The five highest values obtained were averaged, defined as standard for normalization and used to depict all others on a normalized graph with the distance from the nucleus in $\mu \mathrm{m}$ on the $\mathrm{x}$ axis and an averaged intensity of the pixel on the y-axis. The length of the 
analyzed line was converted into $\mu \mathrm{m}$ applying the formula shown in 3.2. by inserting the same values. High average intensity values represent mitochondria at this distance. Subsequently all averaged intensity values of the 39 scans were aligned according to their distance from the nucleus and then averaged. The resulting normalized density graph may be seen as a probability indicator to find mitochondria at a certain distance to the nucleus (Fig. 14). The plot of mitochondrial distance to the nucleus against the normalized density of mitochondria revealed that $50 \%$ of mitochondria are located within $7 \mu \mathrm{m}$ of the nucleus. For better illustration a cumulative density graph was calculated. Taking into consideration that the other $50 \%$ of mitochondria spread out another 13 to $27 \mu \mathrm{m}$ in the cytoplasm the unequal distribution of mitochondria in astrocytes is obvious.

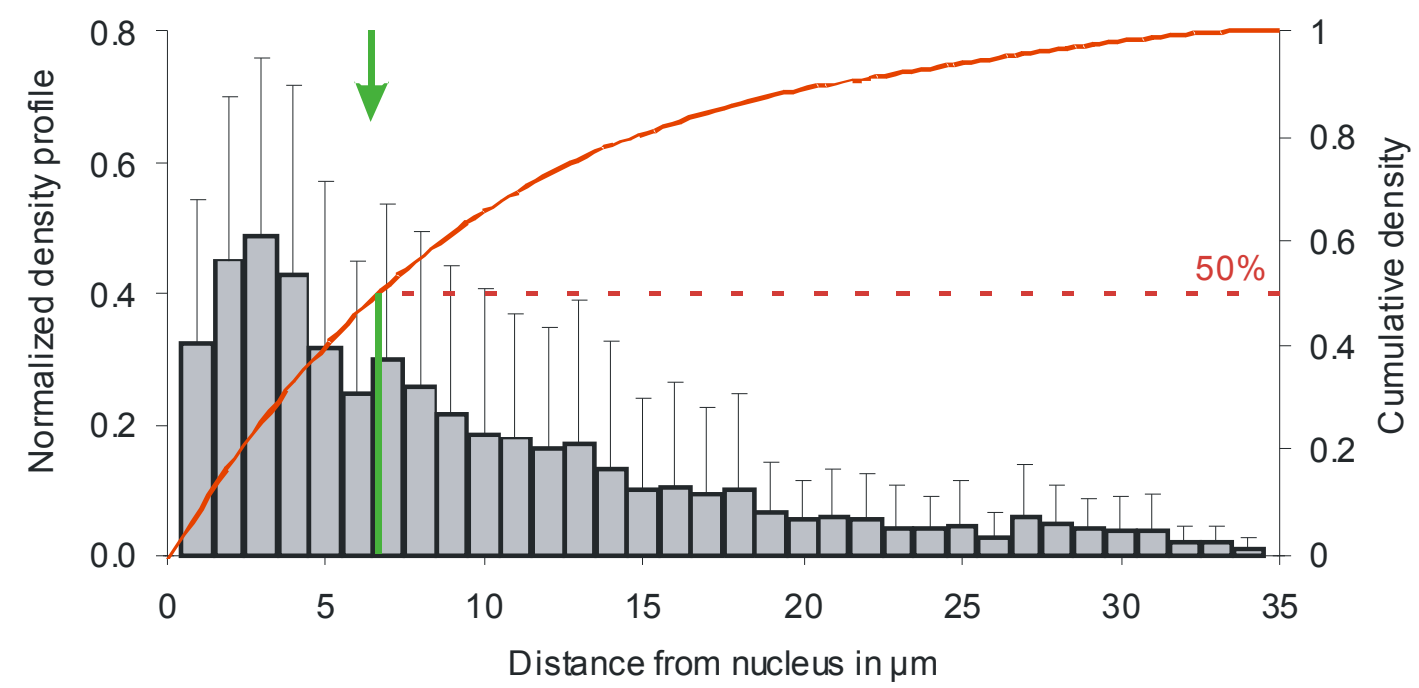

Figure 14 A normalized averaged density distribution of mitochondria from the edge of the nucleus

However, some few astrocytes revealed a rather equal distribution of mitochondria (Fig. 15). More rarely astrocytes showed regions of higher mitochondrial density that were not perinuclear. More often a protrusive area of high mitochondrial density deriving from a perinuclear region of high density of mitochondria was observed (Fig. 16). 


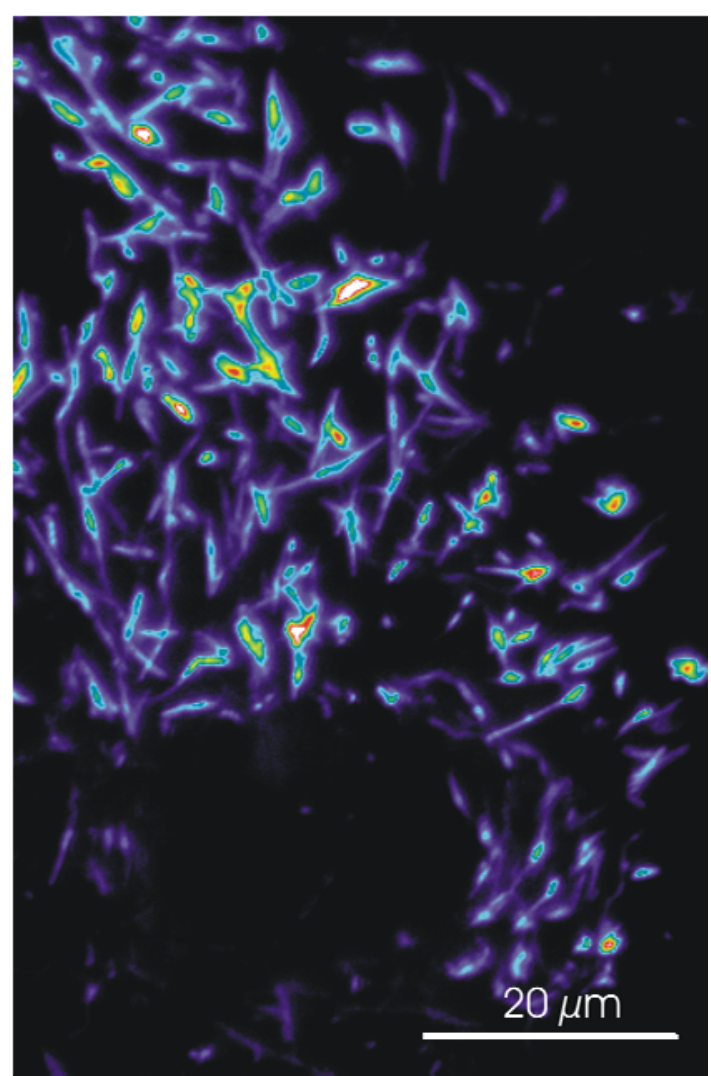

Figure 15

Mitochondria are equally distributed in the cytoplasm of this astrocyte.

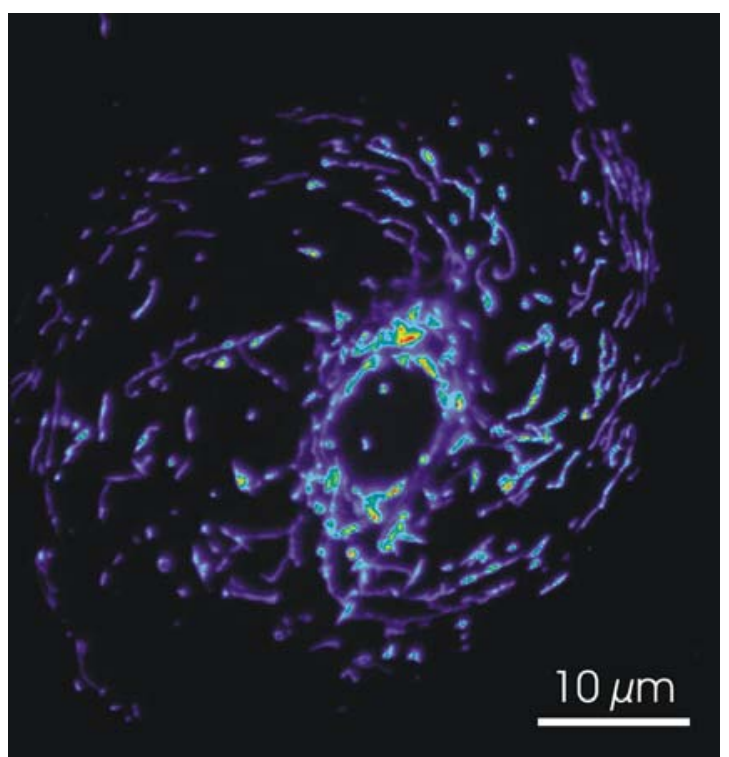

Figure 16

The astrocyte shows spiral-formed protrusions of higher mitochondrial density evolving from the perinuclear area.

\subsubsection{Colocalization of mitochondria with other organelles}

TPELSM at high resolution revealed a close colocalization of Mitotracker Red labelled mitochondria and the DAPI labelled nucleus of astrocytes supporting the results of the earlier density distribution analysis (Fig. 17).

Similarly, a dense clustering of perinuclear mitochondria to the ER, which was labelled with ER Tracker Blue-White DPX, was revealed (Fig. 18). The ER sporadically showed protrusions away from the circular surrounding of the nucleus, which explain why densely packed mitochondria beyond the perinuclear region had been an occasional observation in $\mathrm{JC}-1$ labelled cells.

Mitotracker Red labelling once more confirmed the mostly irregular distribution of mitochondria within the astrocyte. 
Results

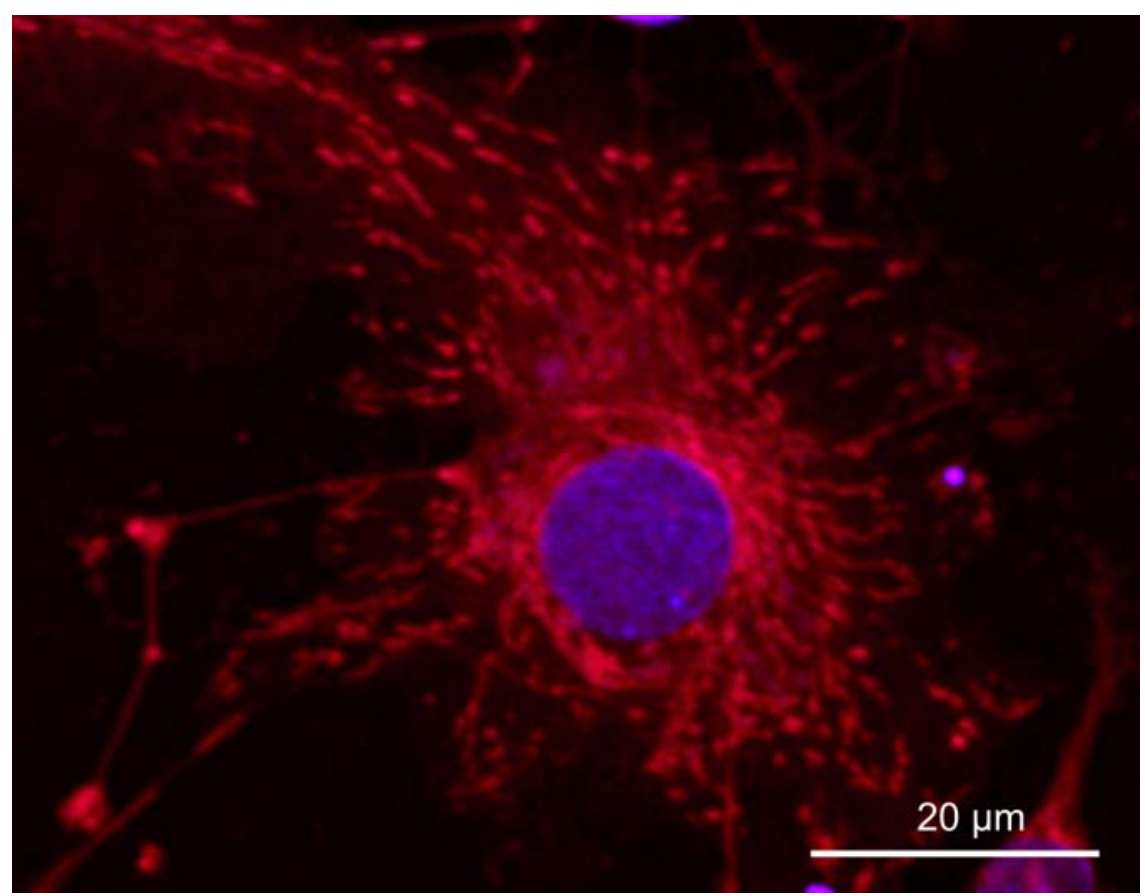

Figure 17

Mitochondria (Mitotracker Red) forming a dense ring around the nucleus (DAPI). (TPELSM scan at 100x zoom)

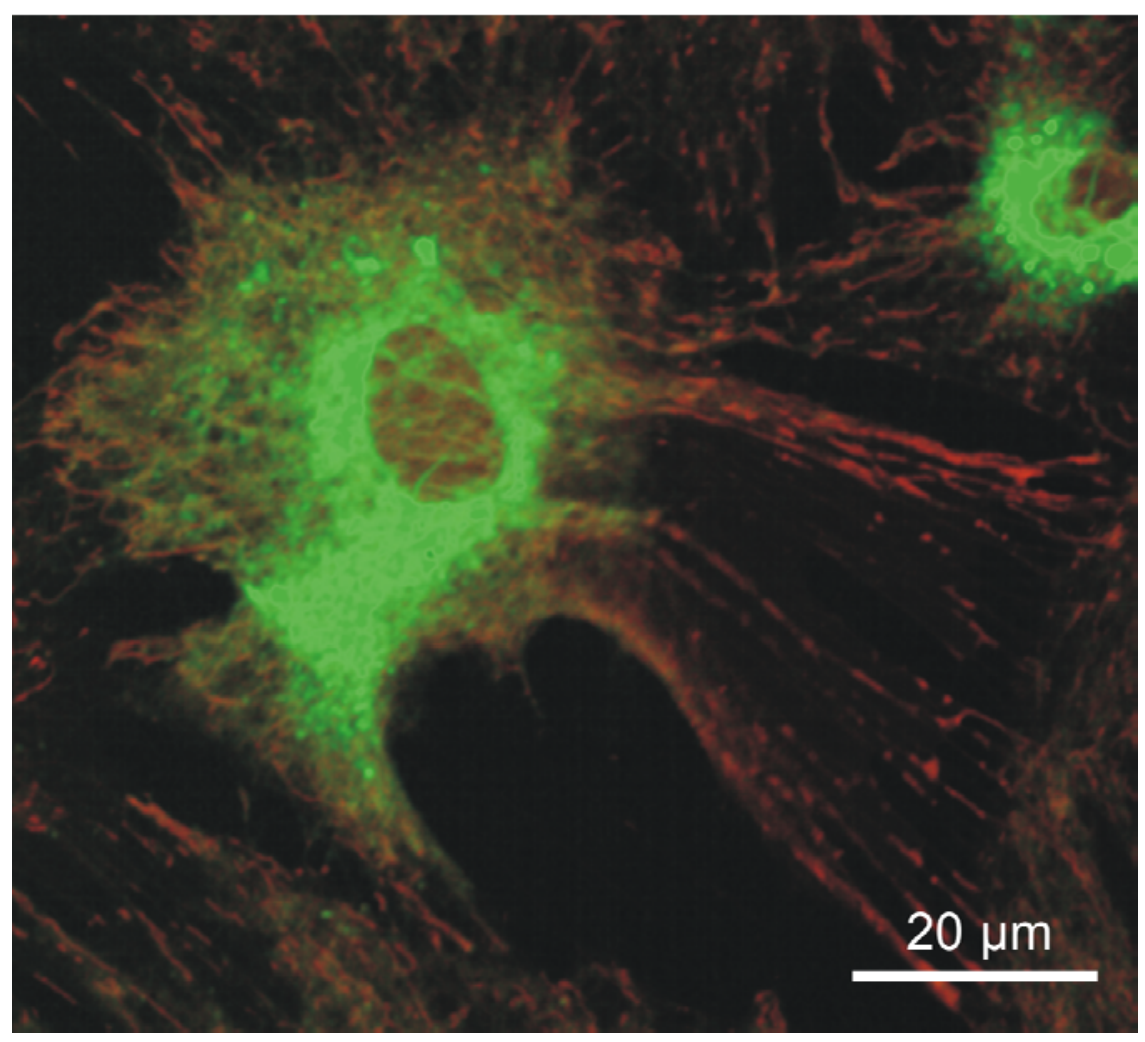

Figure 18

The ER (green) and mitochondria (red-orange) are closely colocalized. (TPELSM scan at $100 \times$ zoom) 


\subsubsection{Observations of mitochondrial movement}

Although movement of mitochondria was not a core aspect of this work the sheer number of observations of mitochondrial movement at haphazard demanded to mention representative examples. There are two main observations. The first is depicted in Fig. 19 showing the coiling of a straight mitochondrion to a grain-like or cup-shaped structure. This is sometimes referred to as a "thread to grain" transition and is a possible consequence of ATP depletion or other respiratory compromise of mitochondria. The usual time course is one minute. I commonly observed this effect - particularly after $\mathrm{CN}^{-}$or FCCP application or after extensive TPELSM scanning.
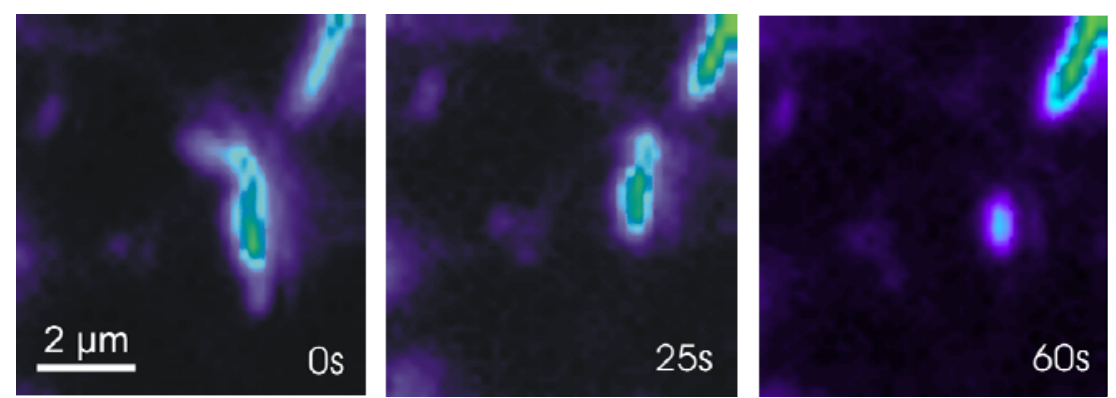

Figure 19 A mitochondrion condensing into a grain-like shape upon repetitive application of glutamate

Another occasional observation was the fast transport of single mitochondria in the cytosol. It was only registered in astrocytes that were not metabolically impaired by pharmacological agents in any way. In particular no directed migration of high-polarized mitochondria to the perinuclear area during glutamate application was observed as had been done before (Diaz et al. 1999). A calculation of one mitochondrion crossing the cytosol resulted in an average velocity of $3.8 \mu \mathrm{m} / \mathrm{min}$. However, no further attempts were made to analyze underlying mechanisms of this transport. Therefore it cannot be stated whether this movement occurred by transportation along cytoskeletal structures or even active movement of the mitochondrion itself. 


\subsection{Variety of $\Delta \Psi_{m}$ in mitochondria}

The heterogeneous polarization of mitochondria was primarily analyzed on mitochondria labelled with the ratiometric $\Delta \Psi_{\mathrm{m}}$ dye $\mathrm{JC}-1$ and was for the aspect of fluctuating $\Delta \Psi_{\mathrm{m}}$ confirmed with Rho123 labelled mitochondria (see 3.3.4.). $\mathrm{JC}-1$ fluorescent molecules are cationic and form J-aggregates in mitochondria that are more polarized than about $-140 \mathrm{mV}$ (Di Lisa et al. 1995). These aggregates emit red light upon excitation, whereas JC-1 monomers, which predominate in less polarized mitochondria, emit green light. This conversion of JC-1 is dynamic and allows a functional analysis of changes in $\Delta \Psi_{\mathrm{m}}$.

For conventional wide-field microscopy the Dual-View ${ }^{\circledR}$ device and a CCD camera were used. This experimental set-up granted high frame rates of 12 per minute and hence a most continuous depiction of changes in $\Delta \Psi_{m}$ in real-time. Higher frame rates of up to 20 per minute were not found eligible due to greater phototoxicity. TPELSM recordings in contrast appealed due to the perfect alignment of the images and excellent contrast. For all analyses only ratiometric images were used to allow conclusions on dynamic changes in $\Delta \Psi_{\mathrm{m}}$.

\subsubsection{An outset analysis of $\Delta \Psi_{m}$}

The digital colour scale applied allowed a limited number of possible intensity values ranging from 0 (minimum) to 255 (maximum) on an 8-bit scale. Ratiometric images must reflect changes in $\Delta \Psi_{m}$ within this scale. Mitochondria analysed for intensity can yield a cumulative distribution graph of $\Delta \Psi_{m}$ (Fig. 20).

It was found important to elicit the a priori state of $\Delta \Psi_{\mathrm{m}}$ for several reasons. First, many of the later experiments focus on effects of pharmacological agents on $\Delta \Psi_{\mathrm{m}}$ in astrocytic mitochondria. An impression of a representative a priori state is utterly important to clearly identify the drug effect. Secondly, long-term recordings (20 - $30 \mathrm{~min})$ without any drug application were run as controls. The a posteriori state after several exposures to UV light allows in contrast 
estimation of phototoxic effects on both JC-1 indicator and $\Delta \Psi_{\mathrm{m}}$.

The analysis of mitochondrial polarization of ratiometric images in 22 astrocytes showed a variability of intensity from 6 to 240 units on the 8-bit scale. The distribution was Gaussian which reveals that polarization is continuous and not clearly divided into two groups of polarized and depolarized mitochondria. The average ratiometric $\Delta \Psi_{m}$ was $84.0 \pm 33.9$ ( $n=1954$ mitochondria) on this scale. Shifts of this mean would represent a ratiometrically expressed polarizing or depolarizing effect on mitochondria.

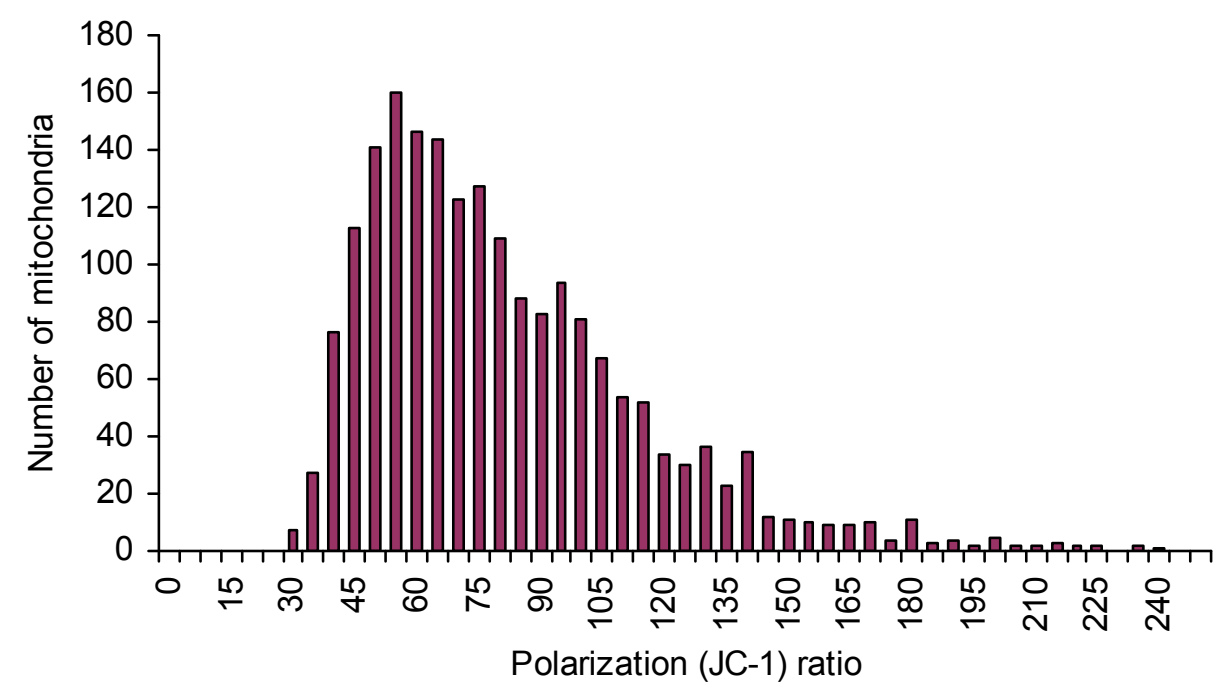

Figure 20 The frequency distribution of ratiometrically analyzed mitochondrial polarization ranged from values between 6 (highly depolarized) to 240 (highly polarized) on a scale from 0 to 255. There is a substantial number of outliers on the highly polarized side.

\subsubsection{Analysis of polarization in respect of mitochondrial length}

A putative correlation of mitochondrial length and $\Delta \Psi_{\mathrm{m}}$ was the first parameter to be analyzed. Those mitochondria, which had already been analyzed for length, were now also analyzed for their average $\Delta \Psi_{\mathrm{m}}$.

1954 mitochondria showed variability in length from 0.3 to $20.2 \mu \mathrm{m}$ and from 6 to 240 bit values of $\Delta \Psi_{\mathrm{m}}$. However, the correlation plot clearly reveals that $\Delta \Psi_{\mathrm{m}}$ is not correlated with the length of a mitochondrial particle (correlation 
coefficient $=0.177$; level of small significance $\geq 0.5$; Fig. 21 ).

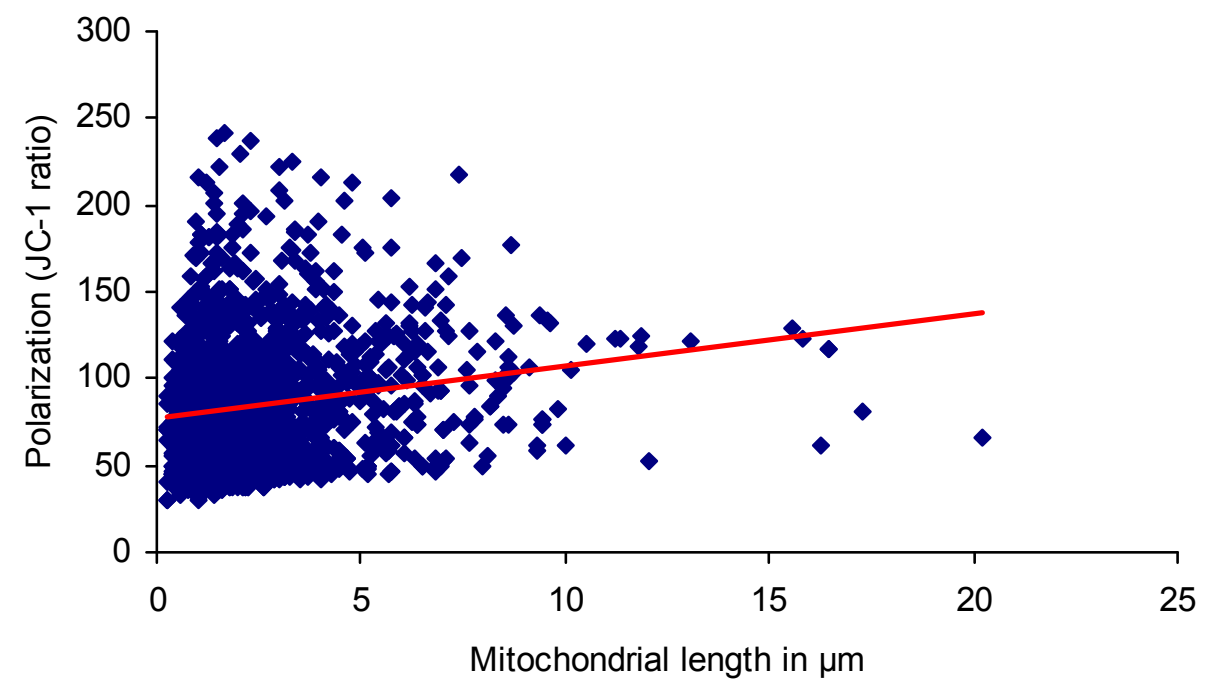

Figure 21 The plot illustrates that mitochondrial polarization and length are independent factors. The trendline shows only poor correlation between them (correlation coefficient $=0.177$ ).

\subsubsection{Analysis of polarization in respect of mitochondrial localization}

If one analyzes JC-1 labelled mitochondria not ratiometrically but only on the red channel, one quickly may get the impression that on average perinuclear mitochondria are more highly polarized. To prove this assumption the intensity values of the mitochondrial regions of the ratiometric images used in 3.2.1. were now correlated with the distance of the mitochondrion to the nucleus. Therefore the centroid $x, y$-values of each region had already been determined in 3.2.1.. Applying Pythagoras' theorem the distance of the centre of a mitochondrion from the estimated coordinate of the centre of the nucleus was calculated in $\mu \mathrm{m}$.

The correlation plot of $\Delta \Psi_{\mathrm{m}}$ and distance to the nucleus revealed that $\Delta \Psi_{\mathrm{m}}$ surprisingly seems not to be affected by the localization of the mitochondrion $(n=1954$; correlation coefficient=-0.03) (Fig. 22). 


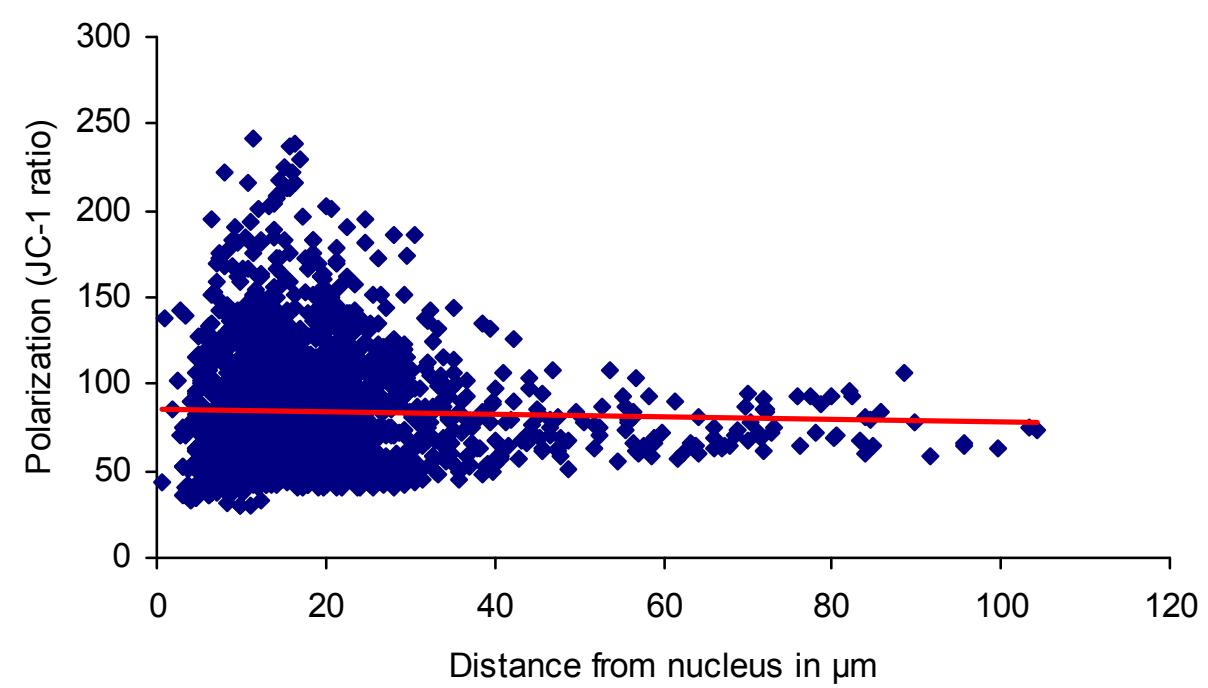

Figure $22 \Delta \Psi_{\mathrm{m}}$ and localization according to this analysis are not correlated in astrocytes at rest (correlation coefficient $=-0.03$ ).

\subsubsection{Spontaneous fluctuations in $\Delta \Psi_{\mathrm{m}}$}

The dynamic state of JC-1 aggregates and monomers made obvious that $\Delta \Psi_{\mathrm{m}}$ is not static but may change more or less regularly over time. This was most clearly seen in the red channel of wide-field microscopic recordings. The phenomenon is often referred to as "blinking mitochondria" or " $\Delta \Psi_{\mathrm{m}}$ fluctuations/oscillations". Putative questions were whether these fluctuations occur instantaneously after the start of recording and if they can be triggered by some chemical agent. Secondly, the percentage of mitochondria with a fluctuating $\Delta \Psi_{\mathrm{m}}$ compared to all mitochondria inside the astrocyte was found worth evaluating. Qualitative aspects of investigation were:

1. Is the fluctuation in $\Delta \Psi_{\mathrm{m}}$ a transient depolarization or higher polarization?

2. How intense is the $\Delta \Psi_{m}$ fluctuation?

3. How long are these episodes of transient changes in $\Delta \Psi_{m}$ ?

4. Are $\Delta \Psi_{\mathrm{m}}$ fluctuations isolated events or is there a spatiotemporal order?

With regard to the spontaneity of fluctuations of $\Delta \Psi_{\mathrm{m}}$ an analysis of $124 \mathrm{JC}-1$ 
labelled astrocytes revealed that in 113 of these cells fluctuations could be observed right from the beginning $(91,1 \%)$, which may give support to the theory that fluctuating $\Delta \Psi_{\mathrm{m}}$ is a normal aspect of vital mitochondria.

As can be taken from Fig. 23 some mitochondria keep a stable $\Delta \Psi_{\mathrm{m}}$ over time whilst others do not. To determine the share of fluctuating, i.e. blinking, mitochondria to the total number of mitochondria present proved difficult.
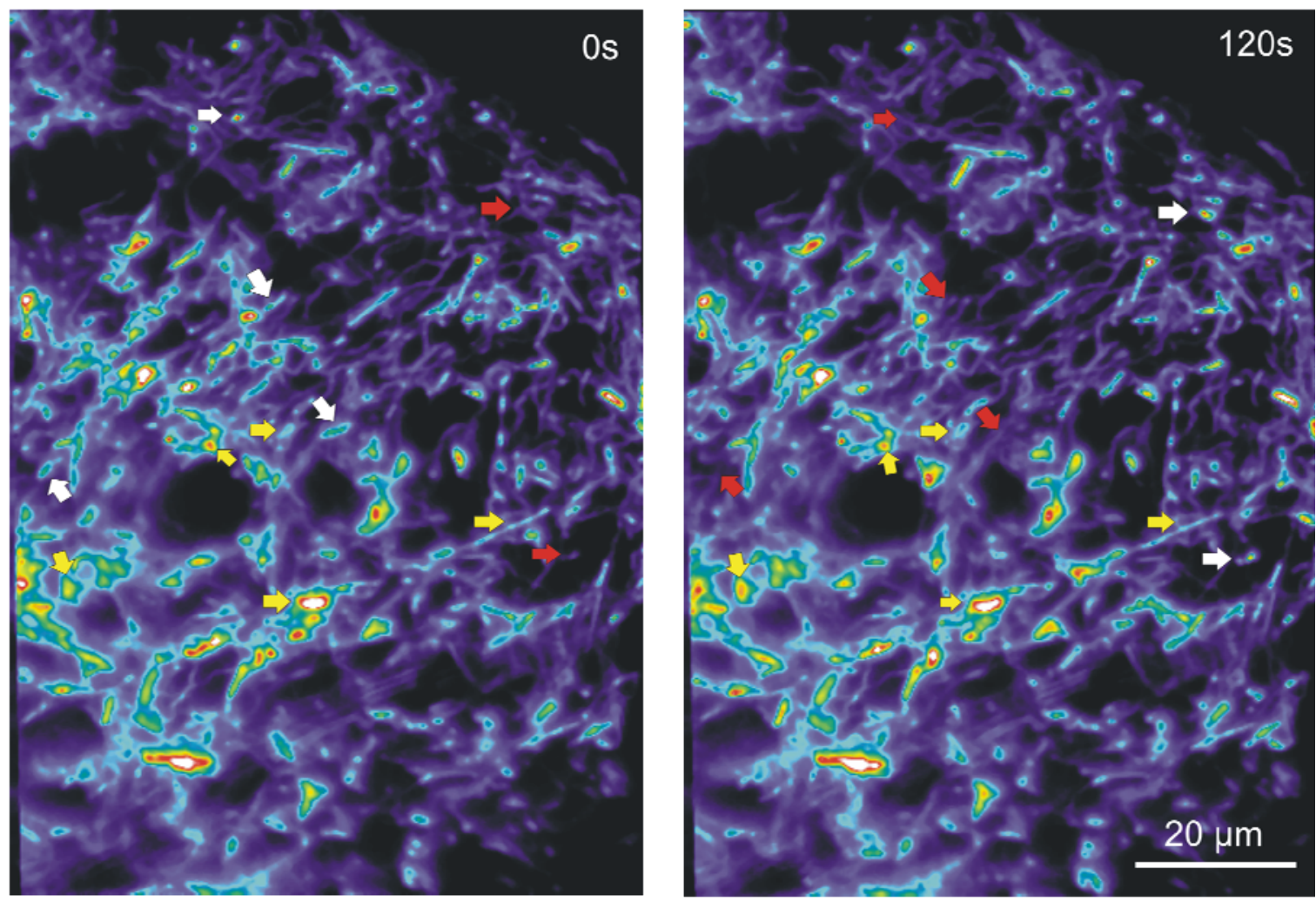

$\Rightarrow$ polarized $\Rightarrow$ depolarized $\Rightarrow$ constant

Figure 23 The image shows that some mitochondria change their $\Delta \Psi_{\mathrm{m}}$ over time whereas others remain at a constant $\Delta \Psi_{\mathrm{m}}$. All images were recorded at a frame rate of $0.2 \mathrm{~Hz}$.

Estimation was tried analyzing ratiometrically 50 mitochondria of an astrocyte with very actively fluctuating mitochondria randomly chosen by threshold function and comparing these with 50 mitochondria of an astrocyte containing apparently more stable mitochondria. In the astrocyte with "active" mitochondria 33 out of 50 mitochondria (66\%) showed at least one $\Delta \Psi_{\mathrm{m}}$ fluctuation during the observation period of three minutes. In comparison, the "passive" astrocyte only contained $26 \%$ of fluctuating mitochondria (13 out of 50 ). These numbers 
estimated probably mark the possible fraction of fluctuating mitochondria within the entire mitochondrial population in an astrocyte at rest.

The result won in this analysis also cleared the question of the direction of polarization: The respective graphs (see Fig. 24) show that mitochondria gain polarization during the fluctuation which may be interpreted as an intermittent state of greater activation of mitochondrial respiration.

JC-1 as a ratiometric probe can be used to convert changes in colour intensity values into approximate absolute voltage values for $\Delta \Psi_{\mathrm{m}}$ applying the Nernst equation as long as the range of intensity priorily was determined applying hyperpolarizing and maximally depolarizing agents, i.e. oligomycin and FCCP for example, to scale the ratiometric intensity values. Although JC-1 was considered superior in a comparative study of ratiometric dyes concerning this estimation (Mathur et al. 2000) I decided to concentrate on relative ratiometric analyses of $\Delta \Psi_{\mathrm{m}}$ both for reasons of practicability during long recordings as well as for doubts about the reliability of the Nernstian approach using potentiometric dyes (Scaduto and Grotyohann 1999).

100 analysed ratiometrically mitochondria reveal normalized fluctuations from baseline (1.0) of $61.0 \pm 44.2 \%$ on average. The maximum value found in all experiments was $3.0(200 \%)$.

Additional analyses were performed on selected mitochondria on separate images showing the same mitochondrion on the red and green channel. Fluctuations of about $150 \%$ were seen in the red channel. Furthermore, here the functionality of the potentiometric probe JC-1 was proven: Corresponding mitochondrial regions show opposite responses in the red and green channel during the $\Delta \Psi_{\mathrm{m}}$ fluctuation (Fig. 24). 


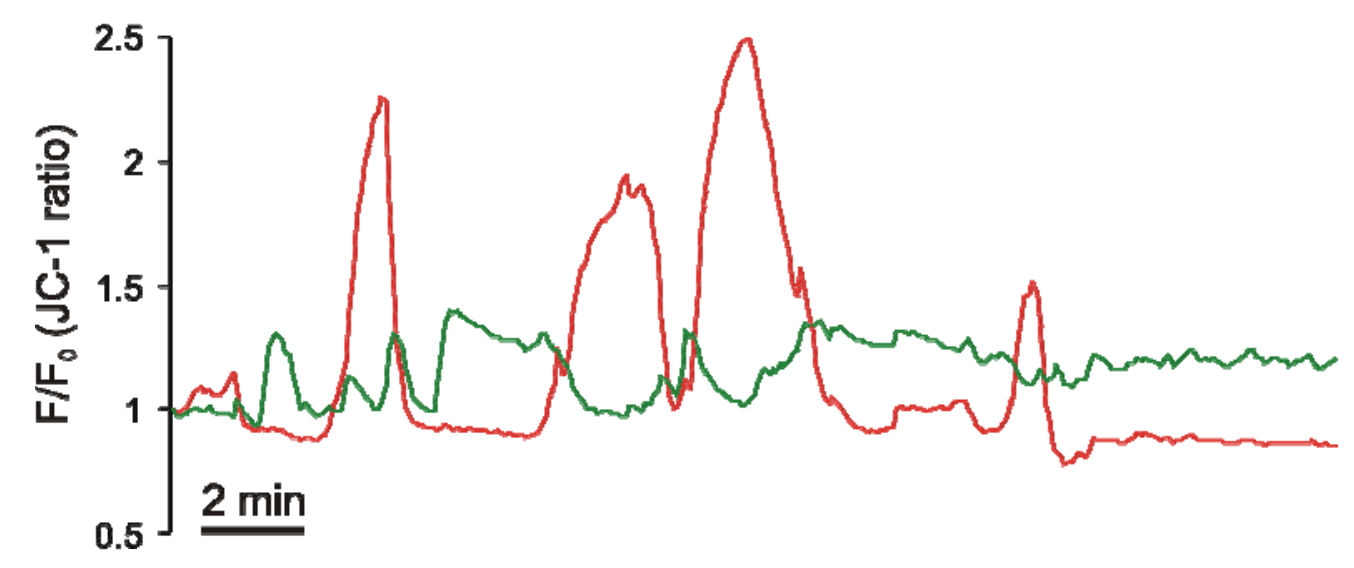

Figure 24 This graph reveals that fluctuations of $\Delta \Psi_{\mathrm{m}}$ occur to turn towards higher polarization - the red channel shows increased normalized averaged intensity. Similarly the green channel decreases in intensity - an indicator to verify that $\mathrm{JC}-1$ molecules indeed reversibly aggregate according to changing $\Delta \Psi_{\mathrm{m}}$.

Another parameter of interest is the time course of the fluctuations. The time course can easily be studied regarding the red channel. The prior analysis of a singular red channel concerning the percentage of mitochondria fluctuating in each population was taken for this purpose and revealed a quite variable duration typically ranging in between $20 \mathrm{~s}$ to $3-4$ minutes.

$\Delta \Psi_{\mathrm{m}}$ fluctuations often seemed to occur randomly. However, due to the fact that 210 experiments were run with $\mathrm{JC}-1$ labelled mitochondria under ideal conditions certain patterns were regularly noticed:

a) Mitochondria sometimes appear to produce synchronized $\Delta \Psi_{\mathrm{m}}$ fluctuations as dense clusters or in close colocalization as can be seen in Fig. 25. This was one of the most regular findings. 


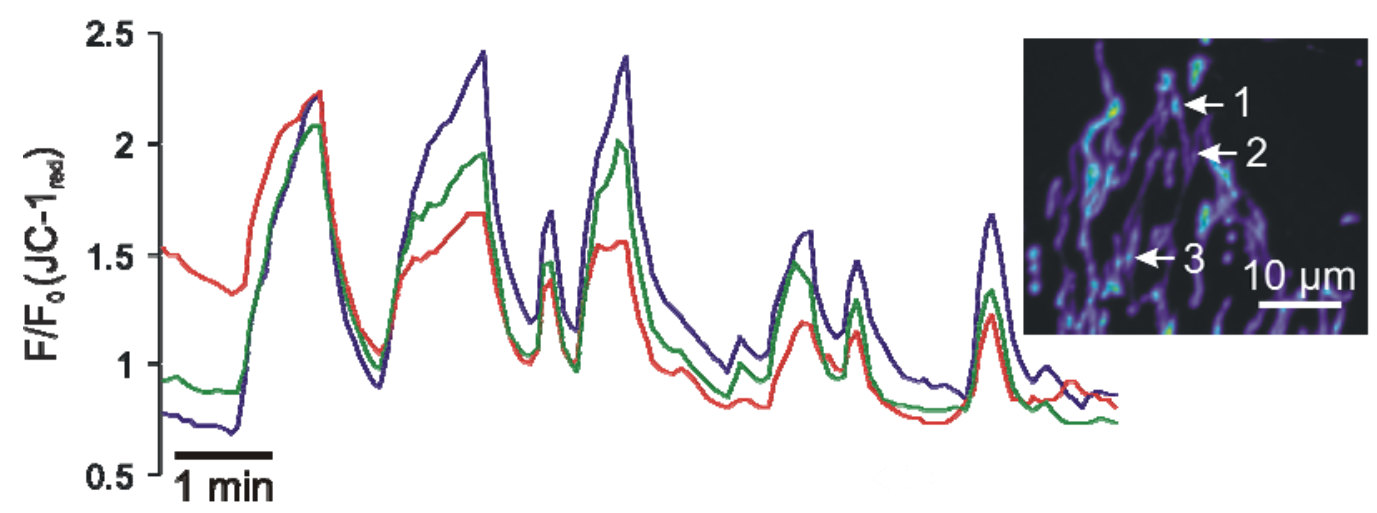

Figure 25 The analysis of normalized average intensity of three adjacent mitochondria revealed a close synchronization of fluctuations in $\Delta \Psi_{\mathrm{m}}$. Similar findings exist for clusters of mitochondria.

b) Some mitochondria only fluctuate once whereas others fluctuate with a high frequency and sometimes over the entire recording period. To find out how long fluctuations persist under control conditions ${ }^{5}, 5$ recordings were carried out showing that $\Delta \Psi_{\mathrm{m}}$ fluctuations continued for at least 18 minutes. The longest persisting fluctuations in a control experiment lasted 24 minutes (Fig. 26). This knowledge is pivotal to discriminate effects of drugs (see 3.4.) from probably phototoxic effects on $\Delta \Psi_{\mathrm{m}}$.

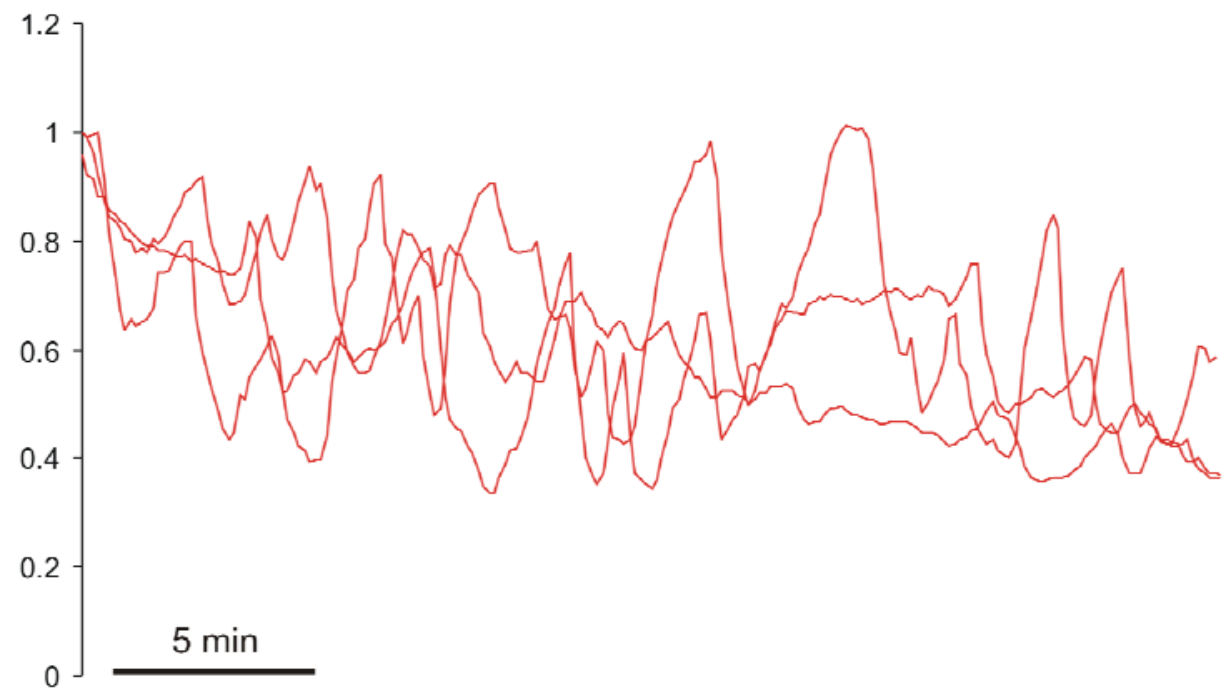

Figure 26 Mitochondria show fluctuations in $\Delta \Psi_{\mathrm{m}}$ for longer than 20 minutes in ACSF.

5 Control conditions mean placement in ACSF at standard temperature and oxygen supply, exposure to $485 \mathrm{~nm}$ light for each time $120 \mathrm{~ms}$ at a frame rate of 12 per minute. 
c) These regional $\Delta \Psi_{m}$ fluctuations could propagate in a wavelike or jumping fashion from one region of higher mitochondrial density to others often adjacent ones (Fig. 27). This finding was rare. In case of a regional propagation an estimated velocity of $3.9 \pm 1.1 \mu \mathrm{m} / \mathrm{s}$ was calculated.

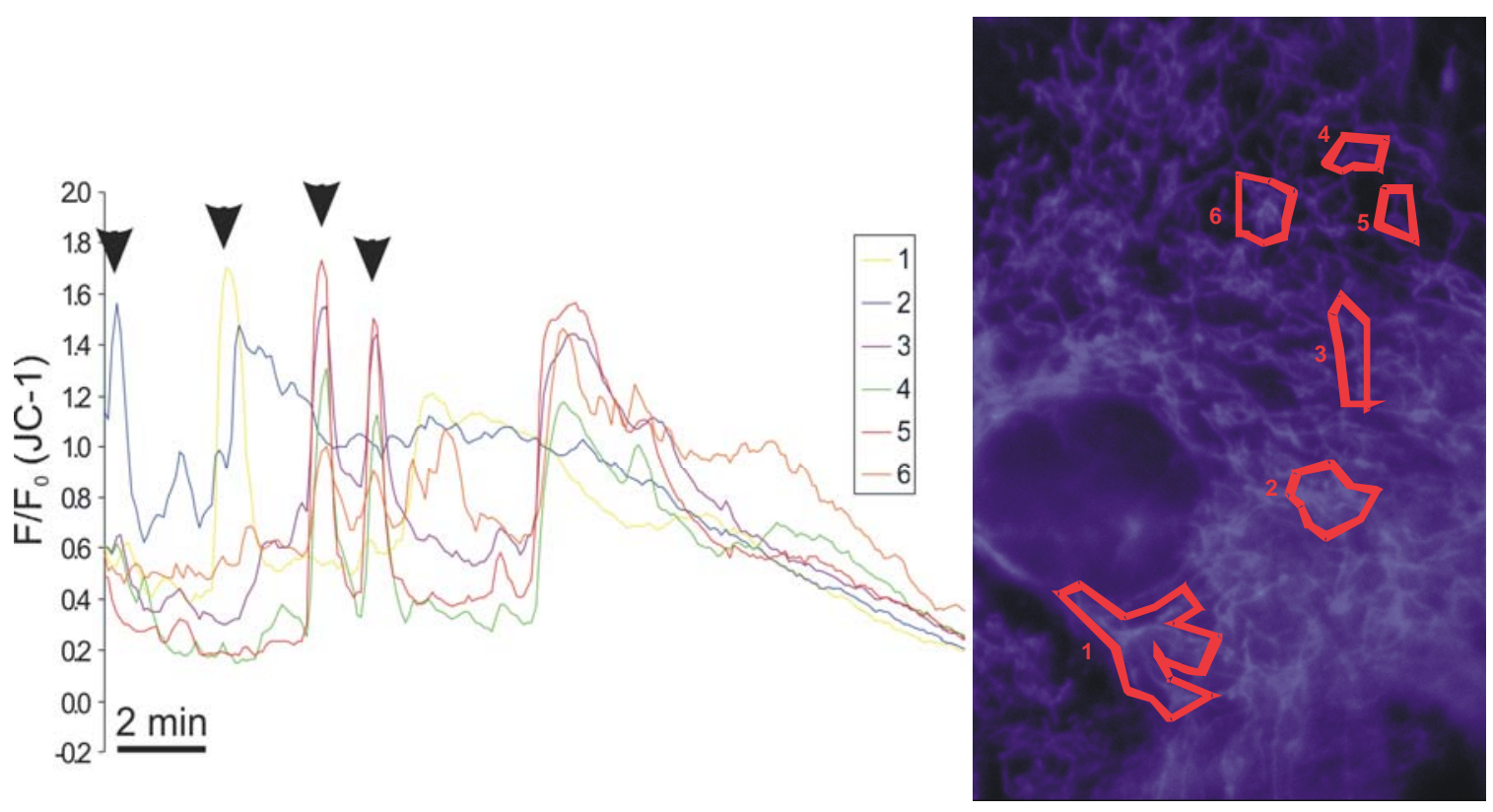

Figure 27 Graph and picture are corresponding. The graph depicts the red JC-1 channels of 6 regions of interest marked in the picture. They show how a rise in $\Delta \Psi_{\mathrm{m}}$ ("spark") travelling between the clusters of mitochondria over time. Note that some of the regions are not very close to each other, e.g. 3 and 6 , and still experience a $\Delta \Psi_{\mathrm{m}}$ rise simultaneously.

d) In several recordings an apparently synchronized or harmonised fluctuation was found in mitochondria that were not close to each other but rather distant (Fig. 27).

JC-1 aggregates apparently are susceptible to acidosis and changes in osmolarity as confounders of the intended ratiometric analyses on $\Delta \Psi_{\mathrm{m}}$ (Duchen et al. 2003). In order to establish a second potentiometric probe that would confirm findings obtained with the indicator JC-1, rhodamine123 (Rho123) was chosen.

Control experiments in ACSF and nominally $\mathrm{Ca}^{2+}$ free ACSF revealed that $\Delta \Psi_{\mathrm{m}}$ fluctuations could equally be observed and also for a similarly long time of 17 
minutes ( $n=10$; Fig. 28). The only difference, which can be deduced from the depicted graphs, is a shorter duration of each fluctuation. This finding may be explained by faster kinetics of the Rho123 molecules, whose fluorescence is varied by a totally different mechanism of quenching at the applied concentration (see 2.4.2.).

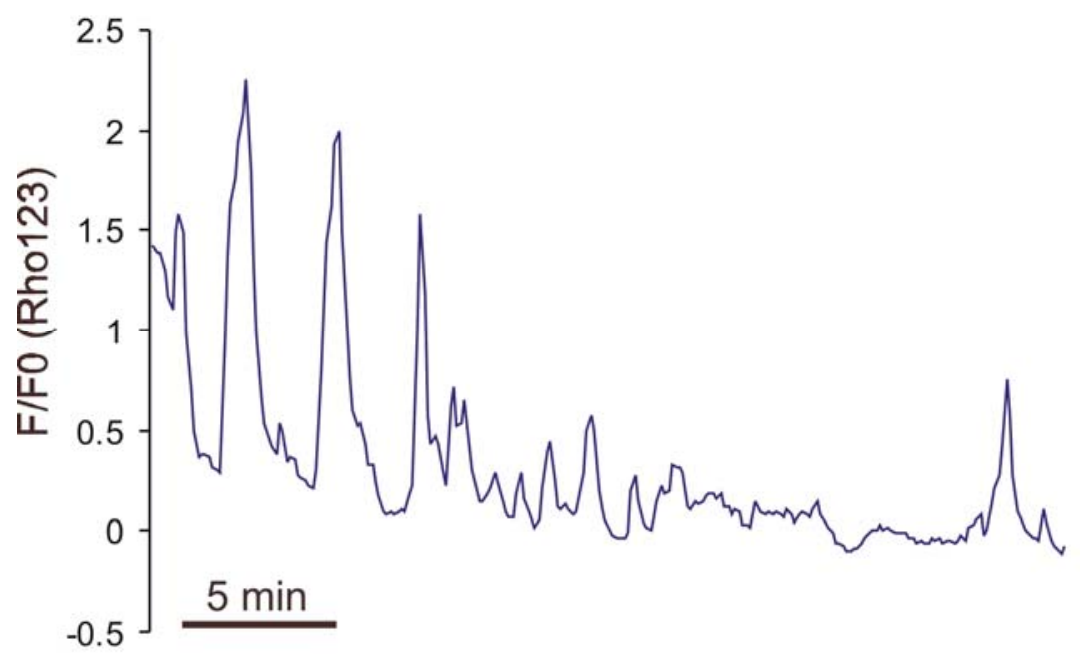

Figure 28 Fluctuations were similarly observed in Rho123 labelled mitochondria. The response kinetics of Rho123 yet appeared to somewhat faster since the frequency of fluctuations was consistently higher.

3.3.5. The influence of $\mathrm{Ca}^{2+}$ ions on $\Delta \Psi_{\mathrm{m}}$ transitions

\subsubsection{Nominally $\mathrm{Ca}^{2+}$ free ACSF in experiments}

Nominally $\mathrm{Ca}^{2+}$ free ACSF was applied to analyze the role of intracellular $\mathrm{Ca}^{2+}$ stores in the propagation of $\mathrm{Ca}^{2+}$ waves (see 3.5.) or fluctuating $\Delta \Psi_{\mathrm{m}}$, e. $\mathrm{g}$. during exposure to suspected modulators of intracellular $\mathrm{Ca}^{2+}$ levels in astrocytes such as dantrolene or 2-APB. It annihilates the influence of extracellular $\mathrm{Ca}^{2+}$ on cytosolic $\mathrm{Ca}^{2+}$ concentration in astrocytes. Usually the cell culture was first placed in ACSF of $1.25 \mathrm{mM} \mathrm{Ca}^{2+}$ and then exposed to $\mathrm{Ca}^{2+}$ free ASCF during the recording prior to drug application. Although this change in ACSF composition has no major effect on ion homeostasis or cellular polarization all astrocytes were given several minute of time to accommodate represented by a stable baseline at a lower level than in ordinary ACSF of an 
observed region of interest (ROI).

Control experiments equal to those with ordinary ACSF concerning fluctuations in 3.3.4. were made. Indeed fluctuations persisted also in nominally $\mathrm{Ca}^{2+}$ free ACSF for at least 18 minutes ( $n=7$; Fig. 29), which demonstrates that external $\mathrm{Ca}^{2+}$ influx is not an important factor in the sustainment of fluctuations in $\Delta \Psi_{\mathrm{m}}$. The average duration of fluctuations was $20 \pm 2$ minutes.

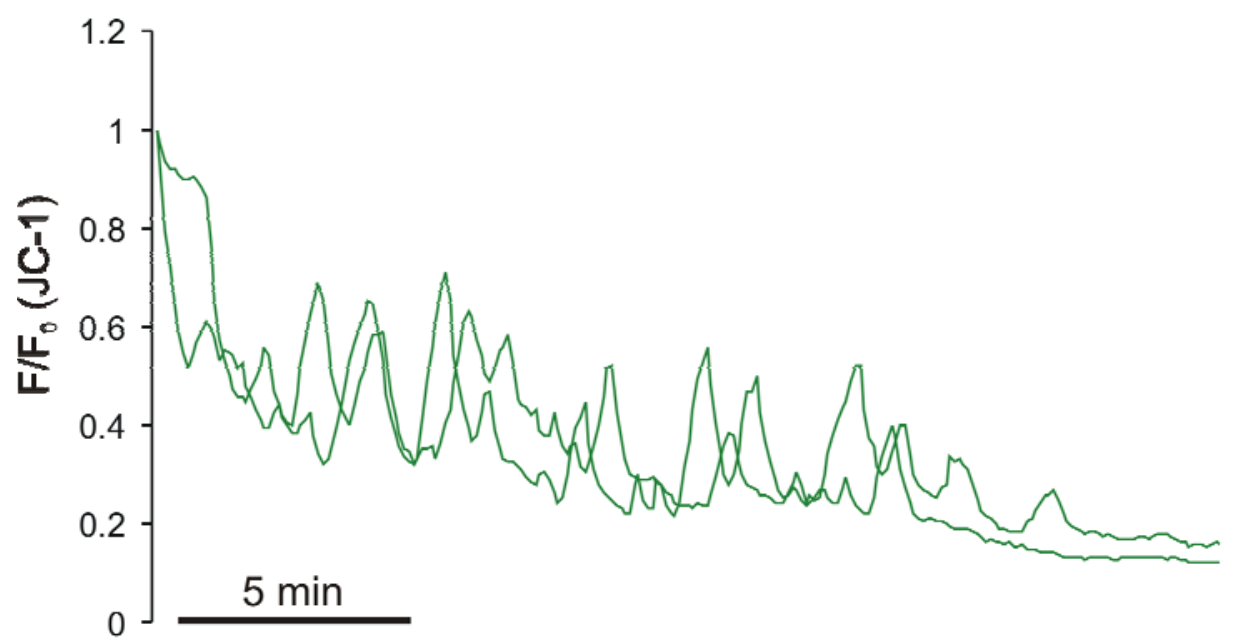

Figure $29 \Delta \Psi_{\mathrm{m}}$ fluctuations persist in $\mathrm{Ca}^{2+}$ free ACSF for a similarly long time as in ordinary ACSF.

\subsubsection{Dantrolene - a blocker of the ryanodine receptor}

In pharmacology dantrolene has long been known as a blocker of the ryanodine receptor (RyR) - a $\mathrm{Ca}^{2+}$ channel present in the endoplasmic reticulum of astrocytes which is responsible for $\mathrm{Ca}^{2+}$ induced $\mathrm{Ca}^{2+}$ release $(\mathrm{CICR})$ to the cytosol (Langley and Pearce 1994).

For experiments with $\mathrm{JC}-1$ labelled mitochondria dantrolene was used to determine the effect of the blockade of $\mathrm{ER}^{\mathrm{Ca}^{2+}}$ release on mitochondrial polarization and $\Delta \Psi_{m}$ fluctuations. This set of experiments was carried in $\mathrm{Ca}^{2+}$ free ACSF and is topically associated with experiments with 2-APB and dantrolene in Fluo-3 AM labelled astrocytes. 
Control experiments with JC-1 labelled mitochondria (see 3.3.5.) showed that $\Delta \Psi_{\mathrm{m}}$ fluctuations would persist in $\mathrm{Ca}^{2+}$ free ACSF for at least 18 minutes. Fluctuations, however, were diminished (in 11 astrocytes) or stopped totally (7 recordings) in 18 out of 22 experiments elucidated the effect of $20-40 \mu \mathrm{M}$ dantrolene during an exposure of 4 to 7 minutes (Fig. 30). In four recordings mitochondria were not affected.

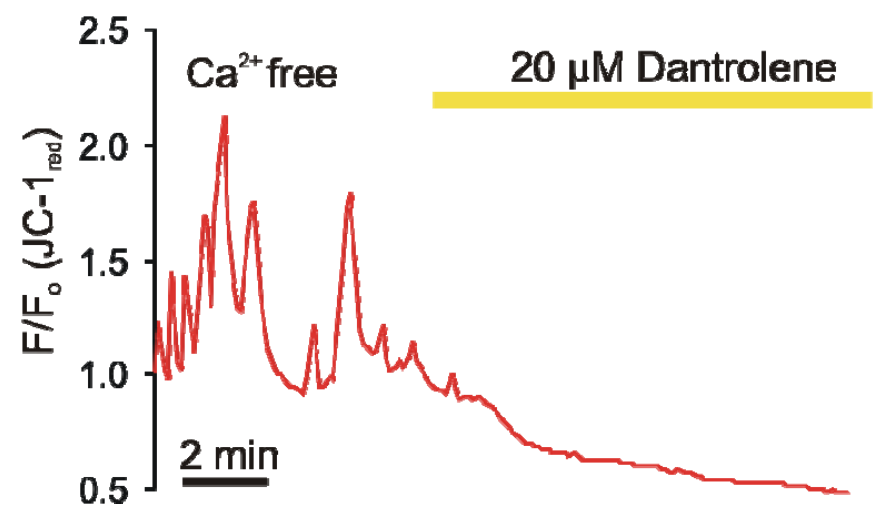

Figure 30 The red channel of $\mathrm{JC}$-1 fluorescence shows a mitochondrion that continues to exhibit a fluctuating $\Delta \Psi_{m}$ during treatment with $\mathrm{Ca}^{2+}$ free ACSF. Fluctuations cease, however, within a few minutes of dantrolene treatment.

Ratiometric TPELSM images taken before, during and after dantrolene exposure also revealed an apparent depolarizing effect of a blockade of RyR dependent $\mathrm{Ca}^{2+}$ stores, which is probably due to competitive absorption of the blocker dantrolene applied, whose intense yellow colour can be expected to interfere with optical recordings (Fig. 31).

This set of experiments pointed at the importance of ER dependent $\mathrm{Ca}^{2+}$ release for both polarization of mitochondria and, more so, fluctuations in $\Delta \Psi_{\mathrm{m}}$. 

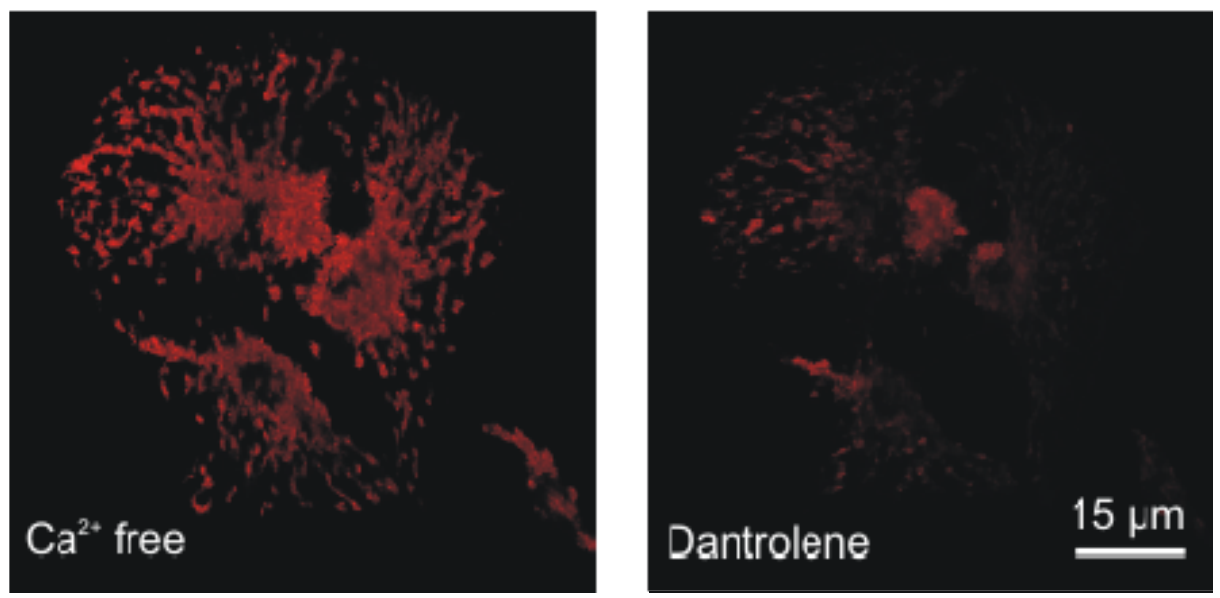

Figure 31 The ratiometric analysis of a set of three astrocytes whose mitochondria were labelled with JC1 reveals that $40 \mu \mathrm{M}$ dantrolene given for 5 minutes depolarize the organelles. Local differences might be found in both the periphery and the perinuclear area (brighter red; i.e. higher polarization). (TPELSM recording at $75 x$ zoom; scaling identical for both images).

\subsubsection{2-APB - a blocker of the $\mathrm{IP}_{3}$ receptor}

2-APB blocks $I_{3}$ receptors on the ER in astrocytes (Milani et al. 1989). In a related set of experiments it was applied at concentrations of $10 \mu \mathrm{M}$ and 100 $\mu \mathrm{M}$ to investigate the extent of $\mathrm{IP}_{3}$ induced $\mathrm{Ca}^{2+}$ release from the ER (IICR) as a source of $\mathrm{Ca}^{2+}$ waves in astrocytes being exposed to nominally $\mathrm{Ca}^{2+}$ free ACSF. Here JC-1 labelled mitochondria were analyzed concerning the influence of the blocker on $\Delta \Psi_{\mathrm{m}}$ and $\Delta \Psi_{\mathrm{m}}$ fluctuations. These experiments were also carried out in $\mathrm{Ca}^{2+}$ free ACSF.

The Dual-View ${ }^{\circledR}$ approach of wide-field microscopy confirmed this effect in realtime. $100 \%$ of astrocytes $(n=15)$ contained mainly depolarized mitochondria after 5 to 8 minutes of exposure to 10 or $100 \mu \mathrm{M} 2-\mathrm{APB} . \Delta \Psi_{\mathrm{m}}$ fluctuations subsided more variably. 14 astrocytes showed mitochondria with primary $\Delta \Psi_{\mathrm{m}}$ fluctuations. Of these mitochondria 6 largely stopped fluctuating after application (Fig. 32), mitochondria of 5 more exposed a decrease in the number of fluctuating mitochondria, 3 astrocytes did not contain mitochondria that were obviously affected by the administration. $10 \mathrm{mM}$ of 2-APB were less and more slowly effective than $100 \mu \mathrm{M}$. 


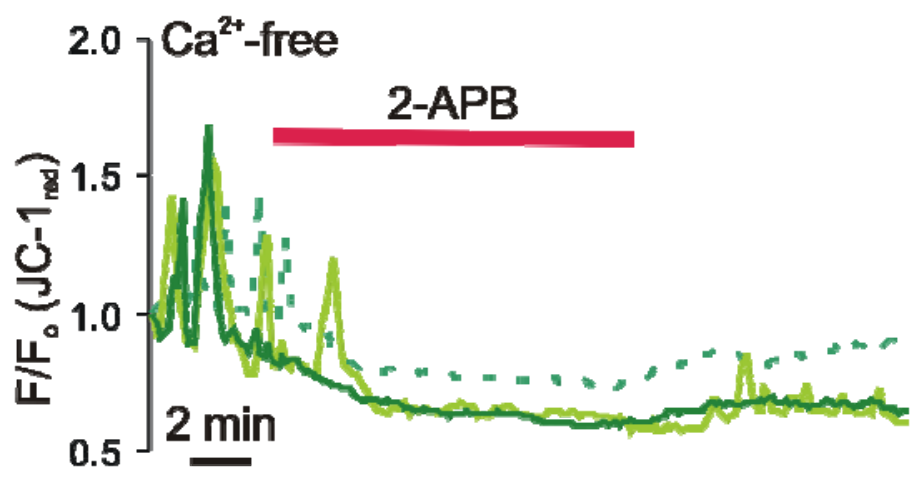

$\mathrm{Ca}^{2+}$ load and metabolic impairment
Figure 32

$\Delta \Psi_{\mathrm{m}}$ fluctuations obviously persist in $\mathrm{Ca}^{2+}$ free ACSF. However, they are blocked by 2APB and scarcely return upon washout. The traces show red channels in $\mathrm{JC}-1$ labelled mitochondria.

3.4. Effects of cellular

In conclusion to the results of 3.3.4. one resumes that fluctuating, sometimes oscillatory, changes in $\Delta \Psi_{m}$ are probably not perfectly arbitrary events inside the mitochondria of astrocytes but underlie cryptic control mechanisms. Since $\Delta \Psi_{\mathrm{m}}$ depends on the metabolic activity of the organelle and is influenced by the intracellular ion homeostasis a large set of experiments was launched to study the effect of several inhibitors of the respiratory chain and modulators of ion, primarily $\mathrm{Ca}^{2+}$, homeostasis. For all experiments only astrocytes with mitochondria of primarily fluctuating $\Delta \Psi_{\mathrm{m}}$ were chosen. Otherwise considerations about the effect of the drug on fluctuations would have been impossible.

\subsubsection{L-Glutamate}

The amino acid is an agonist on ionotropic AMPA and NMDA receptors causing $\mathrm{Ca}^{2+}$ and $\mathrm{Na}^{+}$influx and resulting depolarization. Depending on the extent of stimulus glutamate triggers pro-apoptotic pathways in glia as well as neurones (Matute et al. 2006). Glutamate was applied as an excitoxic stimulator at concentrations of $500 \mu \mathrm{M}$ or even $1 \mathrm{mM}$. The depolarization activates NMDA receptor channels causing unselective influx of cations down the respective concentration gradient (Verkhratsky and Kirchhoff 2007). Glutamate in astrocytes may elevate cytosolic $\mathrm{IP}_{3}$ concentration via activation of 
metabotropic receptors (mGluR) causing $\mathrm{Ca}^{2+}$ release (IICR) (Berridge 1993, Porter and McCarthy 1995).

At a lower concentration of $50 \mu \mathrm{M}$ glutamate proved useful as a viability marker prior to micro-fluorimetric $\mathrm{Ca}^{2+}$ measurements with Fluo-3 AM leading to a short $\mathrm{Ca}^{2+}$ rise not only in vital neurones (Gerich et al. 2009) but also in glial cells.

The application of $500 \mu \mathrm{M}$ or $1 \mathrm{mM}$ showed that in $50 \%(n=16)$ of the astrocytes mitochondria would show a direct depolarization after 4 to 7 minutes of application. Another $25 \%$ would depolarize significantly after a second or third application of the amino acid. There was no difference found concerning the difference in concentration on the effect of glutamate.

$\Delta \Psi_{\mathrm{m}}$ fluctuations greatly diminished or stopped in 5 out of 16 astrocytes during the first application - all of which also showed pronounced depolarization during this course. The mitochondria of the other 7 astrocytes whose mitochondria depolarized also stopped blinking at later drug application (Fig. 33). However, repeated glutamate application had scarcely any effect on $\Delta \Psi_{\mathrm{m}}$ or its fluctuations in the remaining $25 \%$ of astrocytes. In these cases even an initially increasing rate of $\Delta \Psi_{\mathrm{m}}$ fluctuations was observed as well as an increasing number of fluctuating mitochondria.

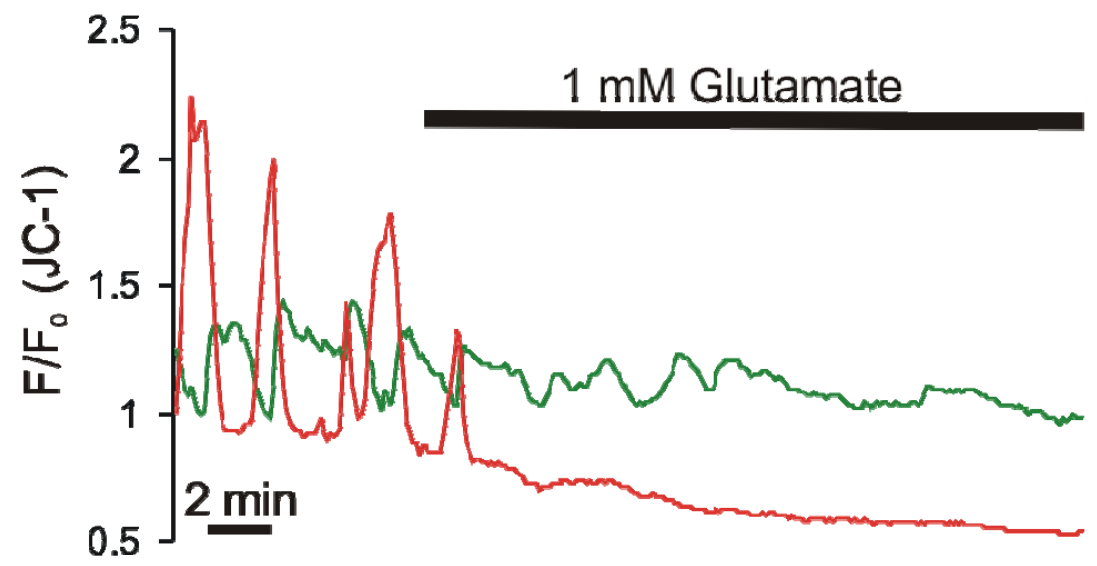

Figure $331 \mathrm{mM}$ Glutamate abolishes fluctuations in $\Delta \Psi_{\mathrm{m}}$ either after the first or after repeated application. 


\subsection{2. $50 \mathrm{mM} \mathrm{K}^{+}$}

$50 \mathrm{mM}$ of $\mathrm{K}^{+}$in the extracellular compartment depolarize astrocytes causing substantial $\mathrm{Ca}^{2+}$ influx into the cell via voltage-gated channels (VGCC). This depolarization is not associated with a major influx of other ions, but provides selective $\mathrm{Ca}^{2+}$ permeability. The concentration is about three times higher than normal buffering loads during increased neuronal activity and rather mimics $\mathrm{K}^{+}$ concentrations found in pathologic conditions such as spreading depression and cytolysis, e.g. during ischemia. Walz et al. showed that high extracellular $\mathrm{K}^{+}$is a stimulus for the astrocytic $\mathrm{Na}^{+} / \mathrm{K}^{+}$-ATPase (Walz 1989).

An exposure to $50 \mathrm{mM} \mathrm{K}^{+}$for 4 to 8 minutes markedly depolarized mitochondria in 8 out of 9 astrocytes observed by wide-field microscopy (Fig. 34). Fluctuations of $\Delta \Psi_{\mathrm{m}}$ in mitochondria of 5 of these cells stopped. In the cell, whose mitochondria had not depolarized, no such effect was observed. The other 3 astrocytes did not contain mitochondria with primary fluctuations in $\Delta \Psi_{\mathrm{m}}$.

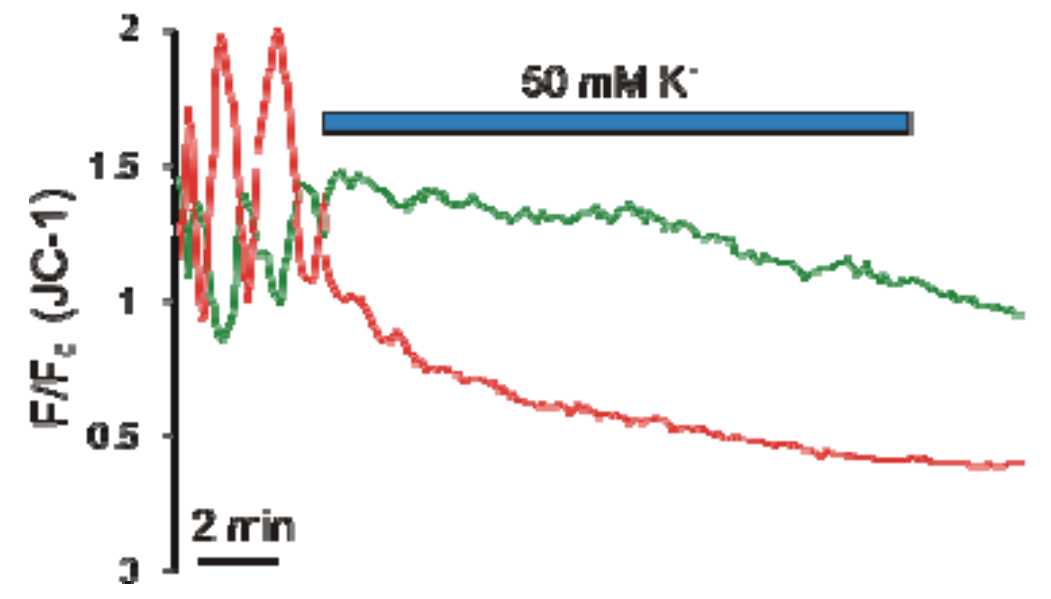

Figure 34 The massive influx of $\mathrm{Ca}^{2+}$ ions due to high extracellular $\mathrm{K}^{+}$causes the depolarization of mitochondria. As can here be seen comparing the red and green traces of a JC-1 labelled mitochondrion depolarization correlates with the termination of mitochondrial $\Delta \Psi_{\mathrm{m}}$ fluctuations.

\subsection{3. $0.2 \mathrm{mM} \mathrm{K}^{+}$}

Härtel et al. discovered that $\mathrm{K}_{\mathrm{ir}}$, a major $\mathrm{K}^{+}$channel involved in astrocytic $\mathrm{K}^{+}$ 
buffering, causes $\mathrm{Ca}^{2+}$ permeability already noted in low $\mathrm{K}^{+}$medium (Hartel et al. 2007). In order to trigger selective and pronounced $\mathrm{Ca}^{2+}$ influx into the cytosol of astrocytes without marked depolarization of the glial cell, the culture was exposed to $0.2 \mathrm{mM} \mathrm{K}^{+}$ACSF for 5 to 8 minutes instead of a regular ACSF containing $3.5 \mathrm{mM} \mathrm{K}^{+}$. The mechanism represents the influence of external $\mathrm{Ca}^{2+}$ influx on intracellular $\mathrm{Ca}^{2+}$ waves (see 3.5.) as well as here on $\Delta \Psi_{\mathrm{m}}$. As far as it is known today no depolarization and influx of ions other than $\mathrm{Ca}^{2+}$ occurs.

Mitochondria in 10 out of 11 astrocytes significantly depolarized during the first application of $0.2 \mathrm{mM} \mathrm{K}^{+}$(Fig. 35$) . \Delta \Psi_{\mathrm{m}}$ fluctuations subsided more variably: In 5 recordings $(n=8)$ they diminished after the first exposure of astrocytes to low $\mathrm{K}^{+}$ ACSF. A second application was necessary in the other 3 . In only 2 of these 8 recordings $\Delta \Psi_{\mathrm{m}}$ fluctuations terminated completely.

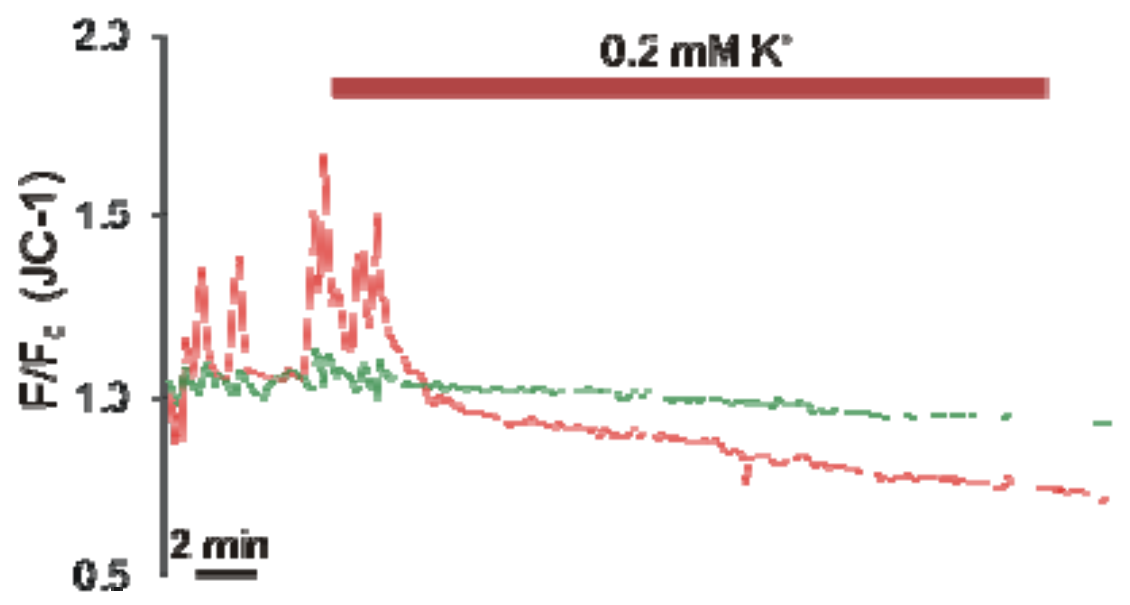

Figure 35 The corresponding red and green traces of a JC-1 labelled mitochondrion illustrate how the organelle depolarizes during exposure of the astrocyte to a low $\mathrm{K}^{+}$environment. As in $50 \mathrm{mM}$ $\mathrm{K}^{+}$experiments, $\Delta \Psi_{\mathrm{m}}$ fluctuations then stop.

\subsubsection{BAPTA}

This chelator of $\mathrm{Ca}^{2+}$ buffers cytosolic $\mathrm{Ca}^{2+}$. The application of a $\mathrm{Ca}^{2+}$ chelator can reveal the dependency on free cytosolic $\mathrm{Ca}^{2+}$ or additionally released from the ER or mitochondrially stored $\mathrm{Ca}^{2+}$ for phenomena such as $\Delta \Psi_{\mathrm{m}}$ fluctuations. 
BAPTA acetoxymethyl ester) accumulated as an ester in JC-1 co-labelled astrocytes.

The combination of BAPTA and JC-1 proved to be difficult for cellular health. However, astrocytes in two recordings seemed to survive the labelling without any impairment (Graph 36). In these cells $\Delta \Psi_{m}$ fluctuations in nominally $\mathrm{Ca}^{2+}$ free ACSF did not stop. The additional application of 2-APB, however, dramatically impaired not only fluctuations but also cellular viability $(n=3)$.

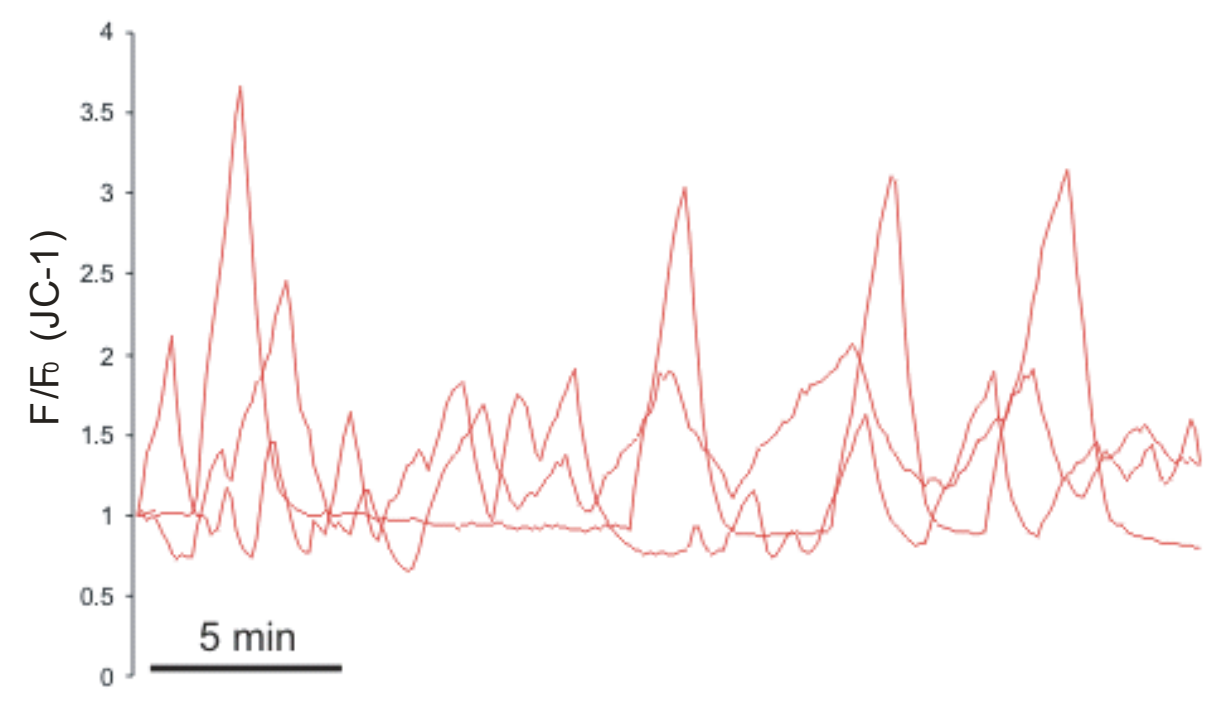

Figure 36 Three red traces of JC-1 labelled mitochondria in a BAPTA loaded astrocyte in $\mathrm{Ca}^{2+}$ free ACSF indicate that normal cytosolic $\mathrm{Ca}^{2+}$ level are not a vital prerequisite to maintain fluctuations in $\Delta \Psi_{\mathrm{m}}$.

\subsubsection{FCCP}

FCCP is a protonophore acting on the inner mitochondrial membrane. The consequent collapse of the proton gradient and therefore $\Delta \Psi_{\mathrm{m}}$ uncouples an unabated electron transport chain from a then impaired ATP synthase (complex $V$ or $\mathrm{F}_{\mathrm{O}} \mathrm{F}_{1}$ ATPase) which in the following runs out of energy for ATP generation. Existing ATP is even hydrolyzed by complex $\mathrm{V}$ running in reverse mode. Mitochondria depolarize rapidly and the $\mathrm{Na}^{+} / \mathrm{Ca}^{2+}$ gradient in the organelle decreases which consecutively impairs the ability of mitochondria to sequestrate $\mathrm{Ca}^{2+}$ (Gunter and Pfeiffer 1990). Repeatedly FCCP was shown to 
increase cytosolic $\mathrm{Ca}^{2+}$ in several cell types (Jensen and Rehder 1991, Luo et al. 1997).

Exposure for 1-3 minutes to $1 \mu \mathrm{M}$ FCCP caused an instantaneous depolarization and end of blinking in the vast majority of mitochondria $(n=3)$ imaged by conventional wide-field microscopy. Some mitochondria stayed polarized against expectation. Both depolarization and fluctuations of $\Delta \Psi_{\mathrm{m}}$ partially recovered after washout of FCCP within several minutes (Fig. 37).

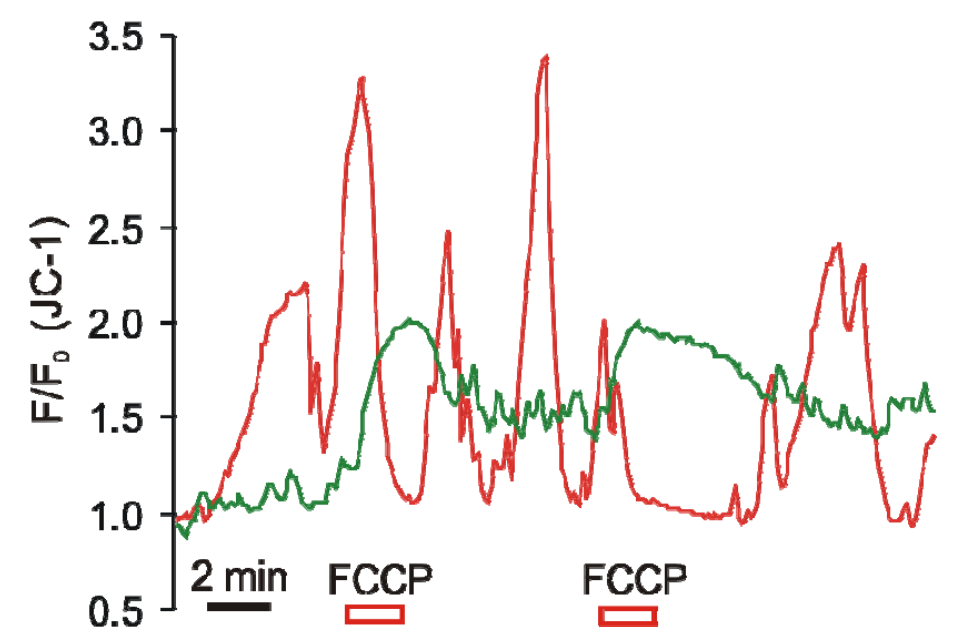

Figure 37 The graph of a red and green JC-1 channel illustrates how the uncoupling with FCCP (application time 90s) depolarizes a normally blinking mitochondrion within seconds. Yet the effect is not lasting since $\Delta \Psi_{\mathrm{m}}$ fluctuations and polarization return. Note that the second application demands a longer phase of recovery than the first.

This finding was confirmed in a TPELSM recording of a short film sequence at a scanning rate of $0.2 \mathrm{~Hz}$. Further TPELSM recordings $(n=3)$ of averaged high resolution scanning sequences before, during and after FCCP application could confirm a depolarization of mitochondria (Fig. 38). 

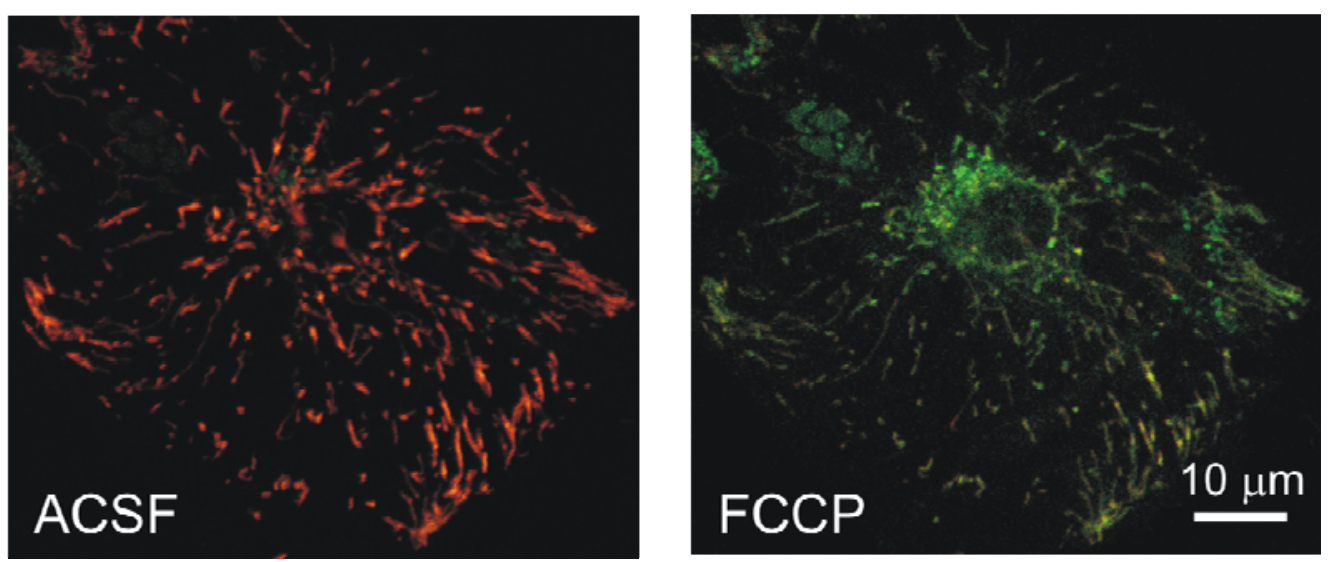

Figure 38 After 2 minutes of $1 \mu \mathrm{M}$ FCCP the overlay TPELSM pictures of red and green JC-1 channels show a distinct shift to the green indicating profound depolarization. Some mitochondria appear to be still more polarized than others - particularly in the cellular periphery.

Another observation was the rapid but reversible occurrence of vesicular structures particularly in the perinuclear region.

\subsubsection{Cyanide}

The complex IV inhibitor cyanide leads to mitochondrial depolarization. It causes channel-mediated potassium release from the cell and sensitization for excitotoxic glutamate as well as a release of cytochrome c - an early step in apoptosis. $\mathrm{CN}^{-}$application is used in a model for hypoxia, since an impaired complex IV means that oxygen can not be utilized for respiration. Furthermore Müller et al. among others have shown in neurons that microtubular transport of mitochondria rests during $\mathrm{CN}^{-}$application (Müller et al. 2005).

The application of this drug for only 1 to 2 minutes at a concentration of $1 \mathrm{mM}$ depolarized most mitochondria in 8 out of 10 recordings. Again, as in FCCP experiments, some mitochondria did not participate in this general trend. In two of the recordings this group of resistant mitochondria was somewhat larger (Fig. 39 and 40). Generally, depolarization did not occur as fulminantly as during FCCP administration. TPELSM recordings confirmed the depolarizing effect of $\mathrm{CN}^{-}(\mathrm{n}=2)$. 


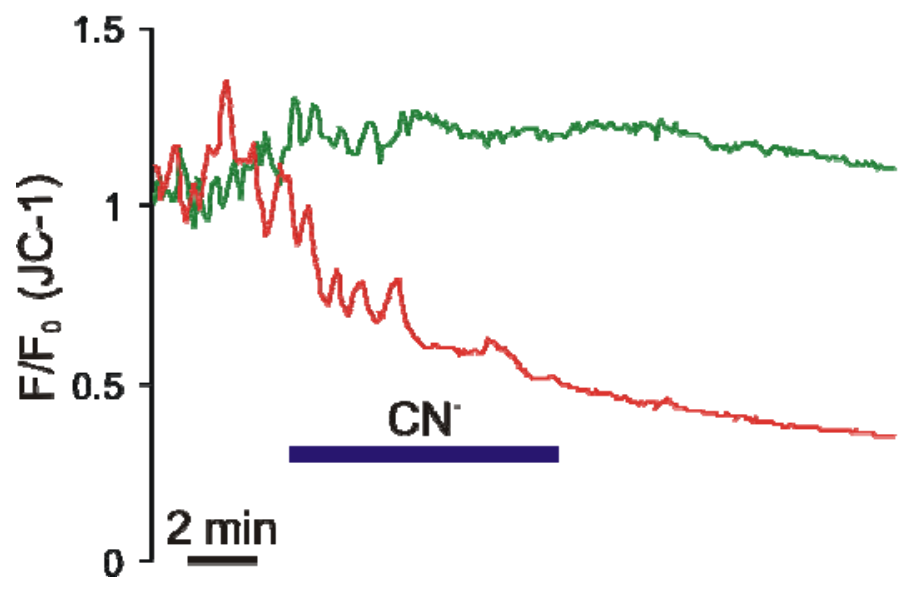

Figure 39 The representative red and green JC-1 traces of a mitochondrion exposed to $1 \mathrm{mM} \mathrm{CN}^{-}$reveals the depolarizing effect of the complex IV inhibitor on $\Delta \Psi_{\mathrm{m}}$. Its effect on $\Delta \Psi_{\mathrm{m}}$ fluctuations is persistent.
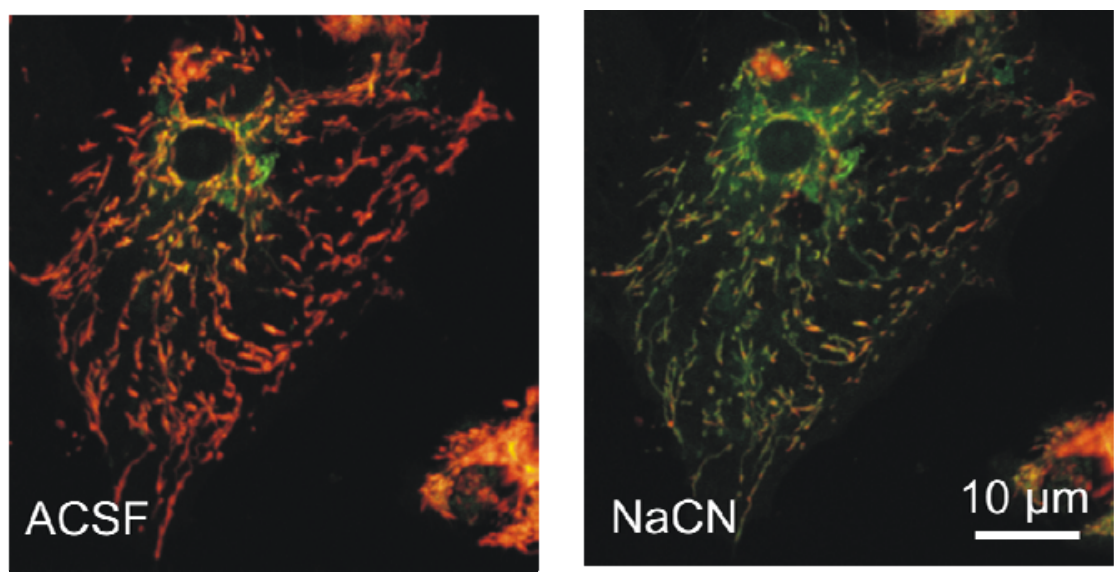

Figure 40 The TPELSM overlay picture of a red and green channel of JC-1 labelled mitochondria reveal the depolarization of mitochondria after 4 minutes of $\mathrm{CN}^{-}$application. Similar to FCCP application peripheral mitochondria appear to be more resistant against the respiratory impairment.

$\Delta \Psi_{\mathrm{m}}$ fluctuations were much less affected by the $\mathrm{CN}^{-}$induced depolarization than caused in response to FCCP: In only 3 out of 12 cases (25\%) they substantially subsided. This finding elicits possibly different mechanisms behind the sustaining of $\Delta \Psi_{\mathrm{m}}$ and fluctuations.

\subsubsection{Azide}

Azide inhibits complex IV similarly to $\mathrm{CN}^{-}$. With regard to neurones it was found 
useful as a model for global ischemia and Alzheimer's disease (Szabados et al. 2004). Neurones from different parts of the CNS were found to react with high variability to equal doses of azide.

At high concentrations of 2 or $4 \mathrm{mM}$ azide applied for about 90 seconds showed a similar effect to $\mathrm{CN}^{-}$on both $\Delta \Psi_{\mathrm{m}}$ and fluctuations ( $\mathrm{n}=13$; Fig. 41). Depolarization was substantial in all astrocytes. Fluctuations of $\Delta \Psi_{\mathrm{m}}$ were diminished in 9 out of 10 astrocytes.

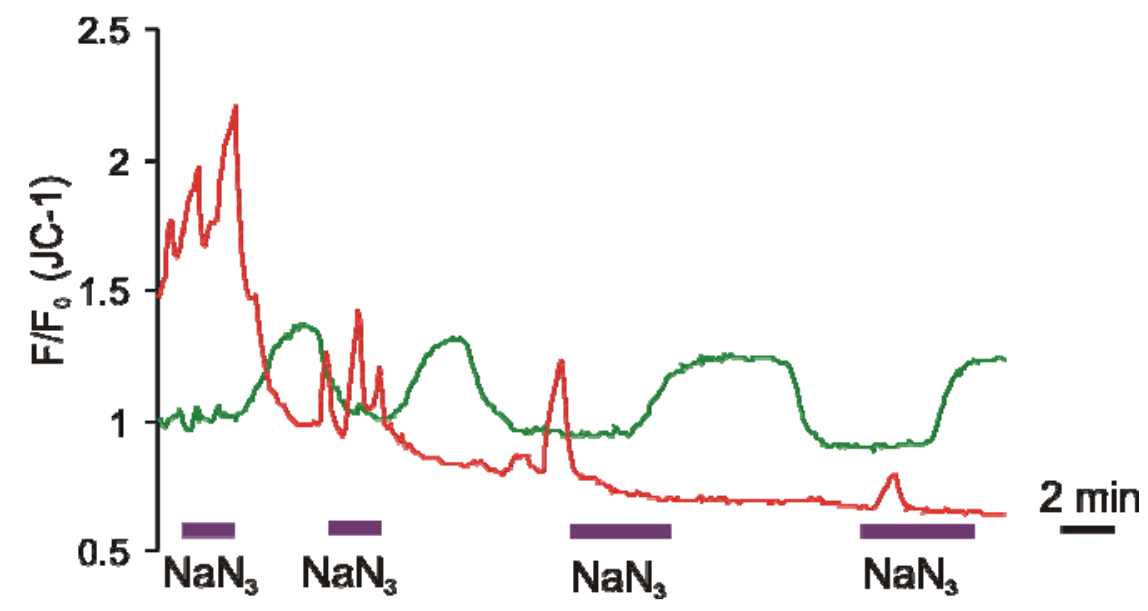

Figure 41 The graph of the red and green JC-1 traces of a mitochondrion recorded with TPELSM almost perfectly depicts how the inhibitor depolarizes mitochondria within 2 minutes after application at $4 \mu \mathrm{M}$. The effect is yet repetitively reversible as fluctuations in $\Delta \Psi_{\mathrm{m}}$ re-establish.

As can be taken from Fig. 41 both polarization and fluctuations did recover to some extent. Ratiometric images of an astrocyte taken before and after the application of $4 \mu \mathrm{M}$ azide clarify that here peripheral mitochondria appear to remain less polarized than perinuclear ones (Fig. 42). 

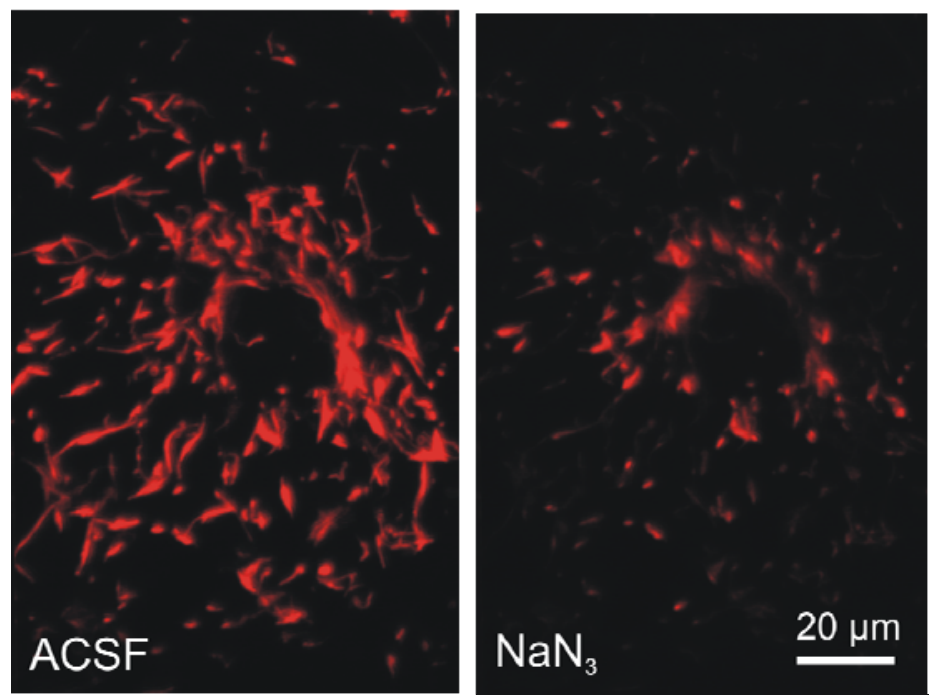

Figure 42 The ratiometric image of a TPELSM recording before and after the application of $4 \mu \mathrm{M}$ of azide reveals that perinuclear mitochondria in this experiment remained more polarized (bright red) than most peripheral ones (dark red). Singular outliers are eminent.

\subsection{Analyses of calcium fluctuations}

\subsubsection{General aspects of $\mathrm{Ca}^{2+}$ transients in Fluo-3 labelled astrocytes}

Experiments with 2-APB, dantrolene, low $\mathrm{K}^{+} \mathrm{ACSF}, \mathrm{Ca}^{2+}$ free $\mathrm{ACSF}$ and BAPTA on JC-1 labelled mitochondria in astrocytes revealed a likely influence of the $\mathrm{Ca}^{2+}$ ion on $\Delta \Psi_{\mathrm{m}}$ and particularly fluctuations in $\Delta \Psi_{\mathrm{m}}$. However, especially the co-ordination of observed wavelike and regional $\Delta \Psi_{\mathrm{m}}$ fluctuations remained in the obscure.

In order to analyze whether cytosolic $\mathrm{Ca}^{2+}$ transients, a phenomenon known for more than 20 years (Berridge and Galione 1988), influence, propagate or trigger fluctuations in $\Delta \Psi_{\mathrm{m}}$, recordings with Fluo-3 AM loaded astrocytes were established to focus on possible similarities of $\mathrm{Ca}^{2+}$ waves and wavelike expressions of $\Delta \Psi_{\mathrm{m}}$ fluctuations. Astrocytes showed two different kinds of transient elevations in cytosolic $\mathrm{Ca}^{2+}$. On the one hand there were local $\mathrm{Ca}^{2+}$ microdomains, which did not propagate across the entire cytosol. On the other hand large $\mathrm{Ca}^{2+}$ waves were recorded (Fig. 43). They moved not only across 
the cytosol of one astrocyte, but also entered neighbouring astrocytes. Both phenomena persisted and were sometimes even stimulated in nominally $\mathrm{Ca}^{2+}$ free ACSF (n=12; Fig. 44).

However, in the vast majority of astrocytes with $\mathrm{JC}-1$ labelled mitochondria fluctuations in $\Delta \Psi_{\mathrm{m}}$ were seen. This was not the case with $\mathrm{Ca}^{2+}$ transients or microdomains in Fluo-3 loaded astrocytes. Rises in $\mathrm{Ca}^{2+}$ were a rare event and would not occur in approximately half of all recordings. The other $50 \%$ would sometimes experience only 1 transient or microdomain for the entire recording although up to 10 events were possible. For $\mathrm{Ca}^{2+}$ transients were often of little intensity and temporally brief, some may have been missed due to the low sampling rate of $0.2 \mathrm{~Hz}$. A usual recording lasted for about 30 minutes.
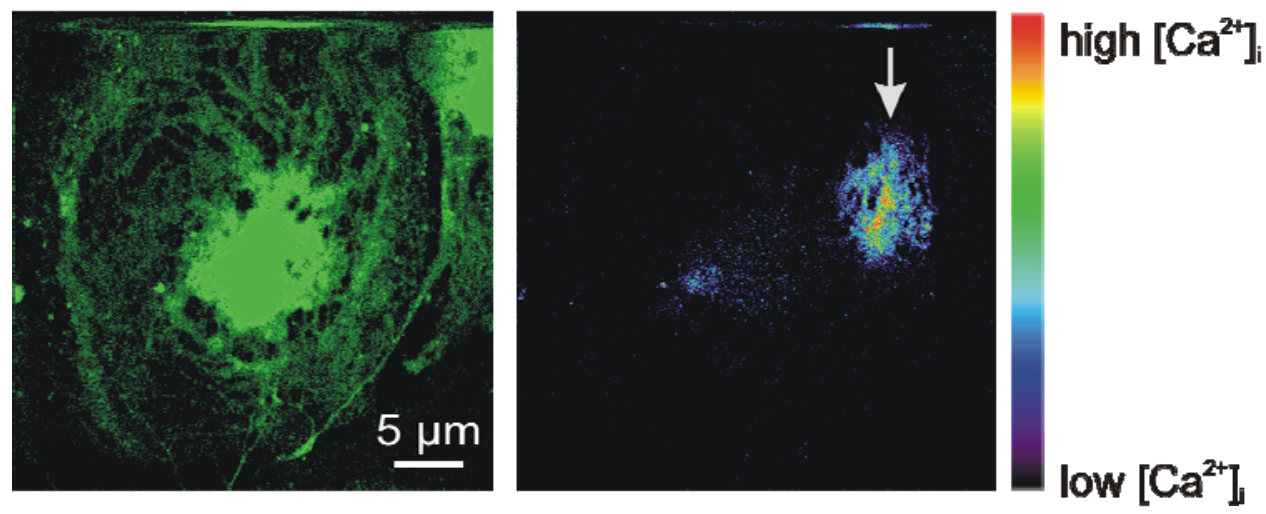

Figure 43 The astrocyte in the left TPELSM picture was loaded with $5 \mu \mathrm{M}$ Fluo-3 AM. Its respective subtraction image shows on the right how a $\mathrm{Ca}^{2+}$ transient regionally emerges from the perinuclear area. Some transients would be able to travel further than the boundaries of one astrocyte.

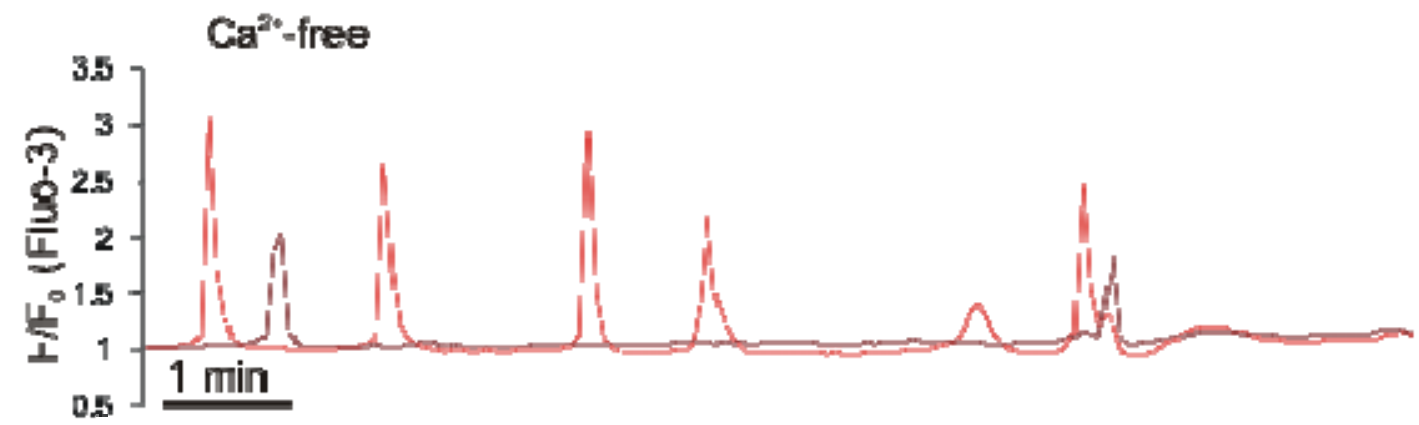

Figure 44 Extracellular $\mathrm{Ca}^{2+}$ is obviously not a necessary prerequisite for the propagation of $\mathrm{Ca}^{2+}$ fluctuations in the cytosol of astrocytes as can be seen by the recurrent fluctuations of up to $200 \%$ in the two analyzed ROI. 
Both $\mathrm{Ca}^{2+}$ waves and microdomains had their individual time course. Microdomains would usually emerge and vanish within 20 seconds of recording. The velocity of travelling $\mathrm{Ca}^{2+}$ waves was calculated for 15 waves occurring in three recordings. The average velocity was $4.4 \pm 3.7 \mu \mathrm{m} / \mathrm{s}$. The fastest wave was $13.5 \mu \mathrm{m} / \mathrm{s}$. These results were obtained calculating the distance travelled by the front of the wave during a certain number of frames, i.e. time period, using the formula of 3.2.1.. The velocity of travelling waves is slightly below the range found by other groups (Dani et al. 1992, Scemes and Giaume 2006), but is similar to the velocity of wavelike propagation of regional $\Delta \Psi_{m}$ fluctuations in JC-1 labelled cells.

A normalized rise in Fluo-3 fluorescence was registered to be up to $300 \%$ but could equally have been only $10 \%$. Fluo-3 fluorescence in microdomains would rise much less. An increase of Fluo-3 fluorescence of $80 \%$ to the baseline was a regular finding.

\subsubsection{Effects of dantrolene and 2-APB}

Blockade of RyR and $\mathrm{IP}_{3}$ receptor dependent $\mathrm{ER} \mathrm{Ca}^{2+}$ sources showed a suppressive effect on $\Delta \Psi_{\mathrm{m}}$ fluctuations. It was now important to elucidate whether dantrolene and 2-APB would have a similar effect on $\mathrm{Ca}^{2+}$ waves and microdomains. The respective experiments were carried out in nominally $\mathrm{Ca}^{2+}$ free ACSF to exclude the influence of extracellular $\mathrm{Ca}^{2+} .40 \mu \mathrm{M}$ dantrolene for 5 to 8 minutes suppressed $\mathrm{Ca}^{2+}$ transients in 9 out of 13 astrocytes (Fig. 45). In 5 out of these 9 cells $\mathrm{Ca}^{2+}$ transients would recover. However, 4 cells showed $\mathrm{Ca}^{2+}$ transients during dantrolene administration. 


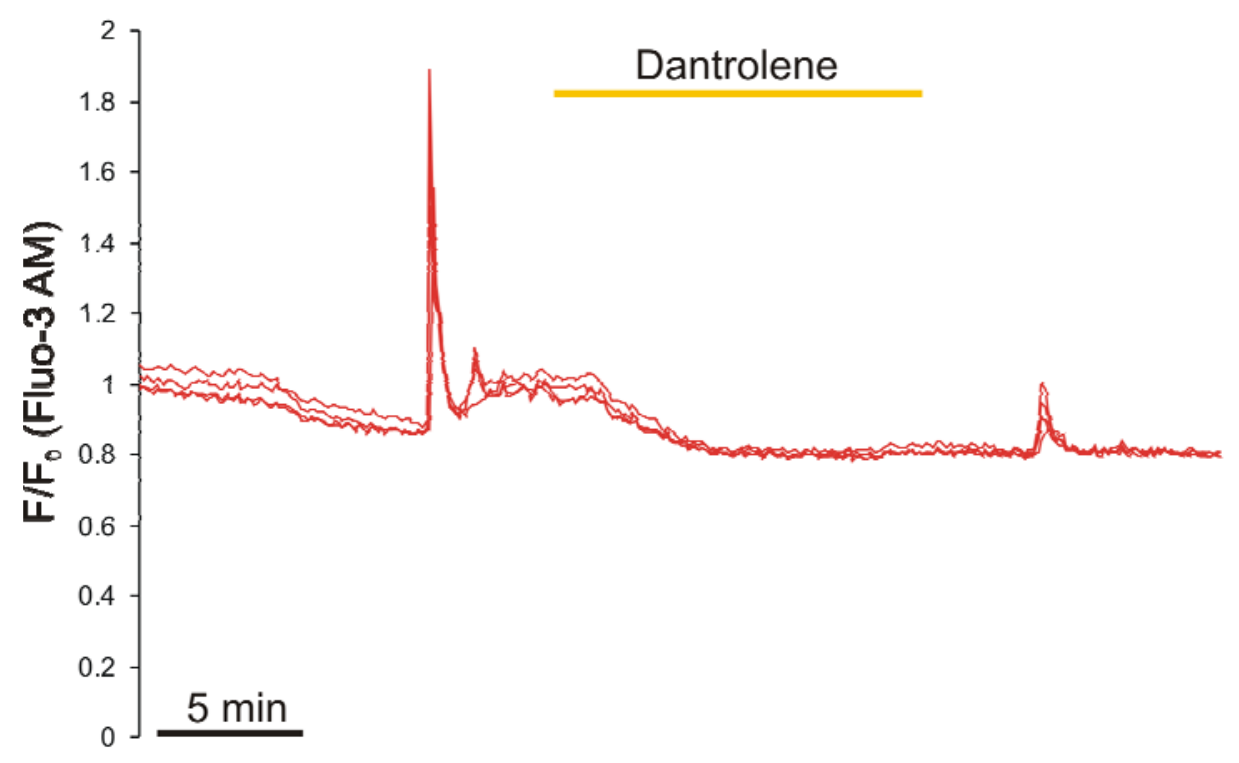

Figure 45 Cytosolic $\mathrm{Ca}^{2+}$ transients were observed regularly in $\mathrm{Ca}^{2+}$ free ACSF. $40 \mu \mathrm{M}$ dantrolene suppressed the $\mathrm{Ca}^{2+}$ transients. In this recording minor transients returned after drug washout.

$100 \mu \mathrm{M}$ of 2 -APB were more effective. 8 out of 10 astrocytes did not express $\mathrm{Ca}^{2+}$ transients during an administration of 4 to 8 minutes (Fig. 46). $\mathrm{Ca}^{2+}$ transients would return in 5 out of these after washout.

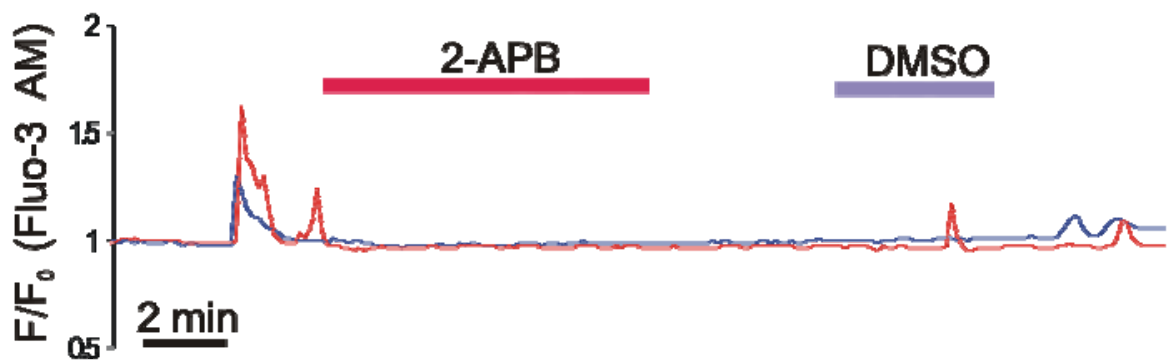

Figure $46100 \mu \mathrm{M} 2-\mathrm{APB}$ proved to suppress $\mathrm{Ca}^{2+}$ fluctuations in most cases. The same recording revealed that $0.1 \%$ DMSO do not affect cytosolic $\mathrm{Ca}^{2+}$ fluctuations - not even after the application of 2-APB. The recording was taken in $\mathrm{Ca}^{2+}$ free ACSF.

These results interestingly are coherent with effects of dantrolene and 2-APB on $\Delta \Psi_{\mathrm{m}}$ fluctuations in $\mathrm{JC}-1$ labelled mitochondria although in $\mathrm{JC}-1$ experiments dantrolene proved to be more potent than in Fluo-3 recordings.

A possible confounder of results was the solvent DMSO that had to be used to 
dissolve several drugs and dantrolene as well as 2-APB in particular. DMSO concentration was always kept below $0.2 \%$ in order to prevent DMSO side effects such as radical scavenging (Repine et al. 1981). However, to exclude such side effects control experiments for both JC-1 and Fluo-3 applying widefield microscopy. DMSO was added to $\mathrm{Ca}^{2+}$ free ACSF in a concentration equal to its concentration when used as a solvent for 2-APB or dantrolene experiments, i.e. $50 \mu \mathrm{l} / 50 \mathrm{ml}(\approx 1: 1000)$. $\mathrm{Ca}^{2+}$ transients persisted unabatedly in all Fluo-3 labelled astrocytes upon 4 to 8 minutes of DMSO application in nominally $\mathrm{Ca}^{2+}$ free ACSF ( $n=4$; see Fig. 46).

Upon the same treatment $\Delta \Psi_{\mathrm{m}}$ fluctuations did not stop neither did mitochondria depolarize in JC-1 labelled mitochondria ( $n=3$; Fig. 47).

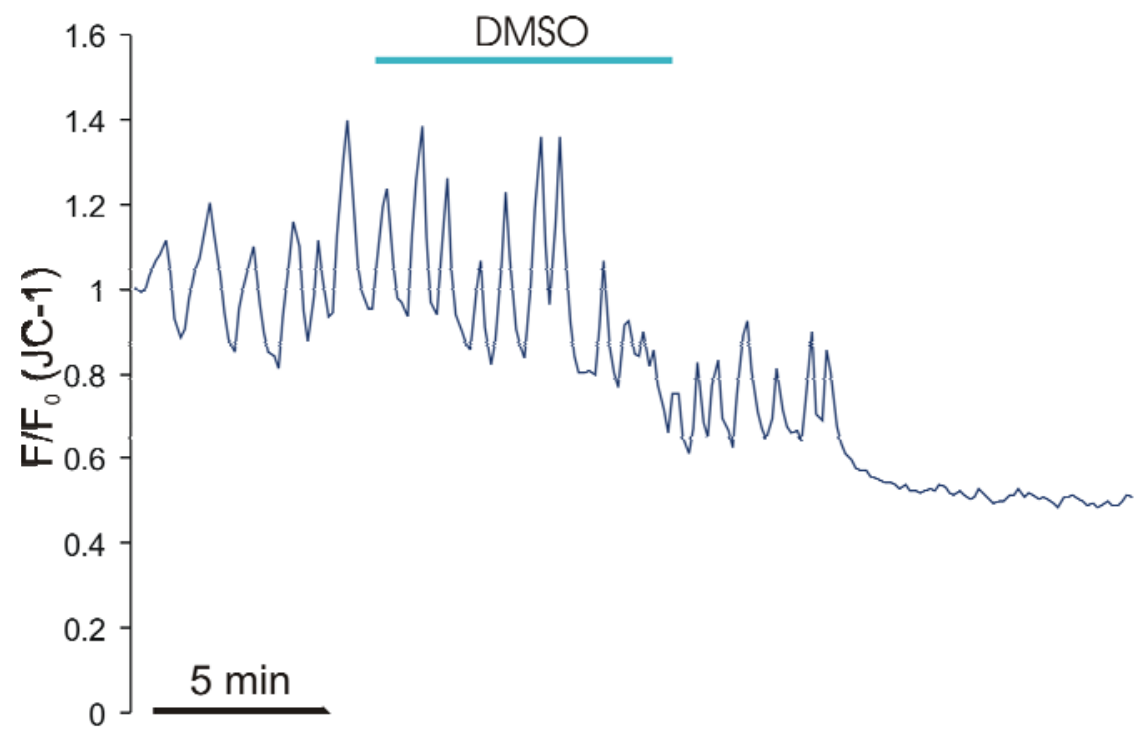

Figure 47 This control experiment proves that $0.1 \%$ DMSO as a solvent for 2-APB and dantrolene did not cause the termination of $\Delta \Psi_{\mathrm{m}}$ fluctuations or depolarization of mitochondria, but rather the agents themselves. The graph depicts a red channel of a JC-1 labelled mitochondrion.

These experiments confirm that DMSO had no effect on $\mathrm{Ca}^{2+}$ transients or $\Delta \Psi_{\mathrm{m}}$. Thus all effects must have derived from the application of the respective drugs. 


\section{Discussion}

The heterogeneity of mitochondria has been a focus in research for the past twenty years and increasingly more functional aspects of their diversity have been revealed (Kuznetsov and Margreiter 2009). Similarly glial cells experienced an eminent revalorization in the concept of CNS function (Ransom et al. 2003). Few publications exclusively elicit the subcellular heterogeneity of mitochondria in astrocytes (Margineantu et al. 2002, Waagepetersen et al. 2006) - a cell type that in one of its classical functions as a provider of energetic substrates to neurons is inextricably associated with the main function of mitochondria as the site of ATP generation. The aim of this thesis is to present for the first time a detailed semi-quantitative and qualitative analysis of both dynamic as well as static aspects of mitochondrial heterogeneity in hippocampal astrocytes on a subcellular level.

The currently most accurate potentiometric fluorophore, JC-1, was established for two comparative imaging methods, classical wide-field microscopy and - for the first time ratiometrically - TPELSM, to determine semi-quantitatively mitochondrial heterogeneity under spatiotemporal aspects. Length, location and distribution of individual mitochondria inside the astrocyte and to each other were juxtaposed to the ratiometric analysis of their $\Delta \Psi_{\mathrm{m}}$. Not only a range of pharmacological tools as a metabolic challenge, but also a complementary potentiometric probe, Rho123, was applied to render a more refined idea of mitochondrial heterogeneity. Furthermore, a novel approach was made to relate these findings to subcellular interaction of mitochondria and the ER. Comparative analyses with $\mathrm{JC}-1$ and Fluo-3 AM labelled astrocytes were executed - the interaction of ER and mitochondria being a current issue in research (Pizzo and Pozzan 2007). 


\subsection{Mitochondrial heterogeneity in morphology and $\Delta \Psi_{\mathrm{m}}$}

The flat aspect of cultured astrocytes with their less densely packed mitochondria allowed analyzing both the individual mitochondrion as well as groups of mitochondria. Although in some cases it was with neither of the two methods used easy to clearly discern one long from two adjacent mitochondria the analysis of mitochondrial length revealed that mitochondria varied greatly in length - the longest analyzed being nearly 50 times longer than the most granular. This finding of a most heterogeneous morphology goes in line with findings of other authors (Diaz et al. 1999, Collins et al. 2002). It is questionable whether filamentous mitochondria differ from granular mitochondria in more than just length. Functional differences were suggested due to a higher occurrence of long filamentous mitochondria in cells with compromised energy metabolism, e. g. in aged cells (Karbowski et al. 1997, Bertoni-Freddari et al. 2003, Martinelli et al. 2003). Long mitochondria probably develop from fusion of shorter ones (Mouli et al. 2009) and disturbance of this process leads to poor cellular health in vitro supporting the idea of functional differences (Chen et al. 2005). Having used astrocytes from DIC 2 to DIC 7 I can, however, not confirm a general tendency of mitochondria to grow longer with more days of the astrocyte being in culture. Secondly, a great variability of lengths was found within the same astrocyte corresponding to the idea of functional heterogeneity of mitochondria on a subcellular level.

Long tubular mitochondria are thought to act as "power wires" that transport $\Delta \Psi_{\mathrm{m}}$ to cellular regions in ATP demand via a network (Skulachev 2001). Higher rates of ATP production influence the concentration gradient of protons across the inner mitochondrial membrane and thus would lead to changes in $\Delta \Psi_{\mathrm{m}}$. My own analysis, however, found no correlation between mitochondrial length and $\Delta \Psi_{\mathrm{m}}$. Reasons for different lengths of mitochondria may thus not necessarily lie in a different respiratory status of these. Another reason could be a fusion of individual mitochondria to allow exchange of mtDNA in order to replace DNA damaged by ROS to regain full functionality (Chan 2006). 
$\Delta \Psi_{\mathrm{m}}$ according to my results was also not affected by distance of the mitochondrion from the nucleus. At the same time mitochondria within one astrocyte were yet heterogeneously polarized. This means that mitochondrial heterogeneity in $\Delta \Psi_{m}$ is not easily determined by mitochondrial localization - or rather colocalization, for it is a wide-spread concept that mitochondria satisfy local demand for ATP (Hollenbeck and Saxton 2005). In studies partially done on cultured astrocytes several authors identified clearly localized regions of higher $\Delta \Psi_{\mathrm{m}}$ for example in the cellular periphery (Diaz et al. 1999, Collins et al. 2002). My results, in partial contrast, do not definitely deny a locally higher mitochondrial activity according to local ATP demand. The continuous and bellshaped distribution of $\Delta \Psi_{\mathrm{m}}$ and multiple ratiometric imaging analyses indeed disclose a most heterogeneous $\Delta \Psi_{\mathrm{m}}$. This may easily represent the mitochondrial response to local ATP demand even though the distribution of highly polarized mitochondria is not territorial but spatially more complicated which might be worth further investigation.

Functional heterogeneity of mitochondria was scientifically proven when finding, e. g., a differential expression of $\alpha$-ketoglutarate dehydrogenase in cortical astrocytes (Waagepetersen et al. 2006) or diverse monoamino oxidase activity in different neurones on a subcellular level (Lai et al. 1994). A first impression during pseudo-colour real-time imaging of the separate red and green JC-1 channels was that mitochondria even show heterogeneity in $\Delta \Psi_{m}$ within one organelle as if being segmented. This observation had been made repetitively in experiments applying JC-1. Common opinion is now though that the aggregation of $\mathrm{JC}-1$ molecules is not exclusively bound to $\Delta \Psi_{\mathrm{m}}$ but that the probability of aggregation is more likely in region with pre-existing J-aggregates, which means that intramitochondrial heterogeneity in $\Delta \Psi_{\mathrm{m}}$ is an artefact. The ratiometric analysis of images supported this finding depicting a much more homogeneous $\Delta \Psi_{\mathrm{m}}$ inside one mitochondrion.

Local heterogeneity in ATP demand may not only be reflected in local 
heterogeneity of $\Delta \Psi_{\mathrm{m}}$, but also in a diverse cytosolic density distribution of mitochondria. Fostering the results of others (Collins et al. 2002) I conclude that mitochondria in astrocytes are obviously not arbitrarily spread, but their density slopes down as the distance to the nucleus increases. A perinuclear dense ring of mitochondria was obvious in most astrocytes which is congruent with the localization of the ER according to my TPELSM analyses using Mitotracker and ER Tracker dyes to label both organelles respectively (see 4.3.), and possibly represents a way of coping with different demand for ATP beyond raising the activity of the individual mitochondrion. The avoidance of a very negative $\Delta \Psi_{\mathrm{m}}$ and thus higher concentration of destructive ROS might be a sensible explanation.

Yet in some astrocytes this more or less obvious observation was not present leading to the question whether these astrocytes are functionally different from those of a different density distribution although otherwise equal in shape and $\Delta \Psi_{\mathrm{m}}$ of their mitochondria. Similarly the analysis of the total cytosolic area filled by mitochondria reveals that astrocytes, partially of the same hippocampus preparation, differ greatly in the density of their mitochondria. It would not be surprising if astrocytes of different mitochondrial density were also functionally different. Experimental analyses concerning the functional and yet not morphological diversity of astrocytes stress this possibility.

\section{2. $\Delta \Psi_{\mathrm{m}}$ fluctuations - from analysis to interpretation}

One of the first and most striking observations during the experiments for this thesis was that a substantial amount of mitochondria does not maintain a constant $\Delta \Psi_{\mathrm{m}}$ for the duration of observation ( 30 minutes). The $\Delta \Psi_{\mathrm{m}}$ fluctuations noticed in both red and green JC-1 fluorescence and ratiometric images were confirmed with Rho123 labelled astrocytes and are thus clearly not an artefact induced by JC-1. It appears that kinetics of $\Delta \Psi_{\mathrm{m}}$ fluctuations vary between the dyes as Rho123 labelled mitochondria fluctuated faster; but this is 
a known experience from experiments with other dyes. $\Delta \Psi_{\mathrm{m}}$ fluctuations were reported first in neuroblastoma cells (Loew et al. 1993) and later also confirmed for mitochondria of astrocytes (Belousov et al. 2001).

$\Delta \Psi_{\mathrm{m}}$ fluctuations were proposed to be most likely a pathophysiological phenomenon (Vergun et al. 2003). I therefore intended both to elicit quantitatively some characterics of $\Delta \Psi_{\mathrm{m}}$ fluctuations as well as find out more about their mechanism. The method of online ratiometric analysis of $\Delta \Psi_{\mathrm{m}}$ combined with two-photon excitation microscopy to my knowledge is novel for the elucidation of this question.

$\Delta \Psi_{\mathrm{m}}$ fluctuations are in fact a repetitive change in activity of the respiratory chain and thus the extent of ATP and ROS production. It was first important to analyse whether mitochondria intermittently decrease or increase oxidative phosphorylation, i.e. ATP generation. Consistently my experiments showed that they increased respiration during a fluctuation, while in contrast another group detected $\Delta \Psi_{\mathrm{m}}$ fluctuation towards depolarization (Collins et al. 2002). Also it is interesting that despite of most astrocytes containing fluctuating mitochondria, not only the share of fluctuating compared to all mitochondria is largely variable, but also that there is no obvious reason why adjacent mitochondria show a different behaviour. For neuronal mitochondria $55 \%$ as a share of fluctuating mitochondria had been reported, which is in line with my findings in glial cells. However, in astrocytes - except inconsistently for glutamate experiments - I never saw the share of fluctuating mitochondria increase - only mitochondria with primarily instable $\Delta \Psi_{\mathrm{m}}$ would subside (see also 4.3.). It is thus possible that either mitochondria or the astrocytes observed are of different subtypes increasing once more aspects of mitochondrial heterogeneity.

As for the static analysis of $\Delta \Psi_{\mathrm{m}} \mathrm{I}$ attempted to find possible similarities of fluctuating and stable mitochondria. In some recordings it was evident that $\Delta \Psi_{\mathrm{m}}$ fluctuations follow a certain spatiotemporal scheme in some mitochondria. First, 
closely entwined mitochondrial clusters often fluctuated simultaneously. A possible explanation may be a chemical communication. Secondly, fluctuations could occur simultaneously in regionally close, yet not adjacent, mitochondria and propagate regionally. $\Delta \Psi_{\mathrm{m}}$ fluctuations were found to be triggered by increased cytosolic $\mathrm{Ca}^{2+}$ levels. Supposing this as one possible way of wavelike $\Delta \Psi_{\mathrm{m}}$ fluctuation propagation I was aiming to identify the possible sources of $\mathrm{Ca}^{2+}$, i.e. the extracellular space, the ER and mitochondria.

\subsubsection{The crosstalk of mitochondria and the ER}

Fluo-3 loaded astrocytes regularly showed either minor microdomains of $\mathrm{Ca}^{2+}$ increase, often in the perinuclear region, or a substantial $\mathrm{Ca}^{2+}$ rise of a $\mathrm{Ca}^{2+}$ wave propagating across the entire cytoplasm and sometimes to adjacent cells. Both phenomena though occurred less often than $\Delta \Psi_{\mathrm{m}}$ fluctuations and also showed different spatial dynamics. On the other hand there are strong similarities between them. First, both $\Delta \Psi_{\mathrm{m}}$ and $\mathrm{Ca}^{2+}$ waves did not depend on extracellular $\mathrm{Ca}^{2+}$ and were not affected by its absence: They depend on intracellular signals. Secondly, experiments with dantrolene and 2-APB annihilated both phenomena equally successfully. The fact that both $\Delta \Psi_{\mathrm{m}}$ fluctuations and $\mathrm{Ca}^{2+}$ waves depend on RyR and $\mathrm{IP}_{3}$ receptor dependent $\mathrm{Ca}^{2+}$ release from the ER reveals that these phenomena derive from a common trigger. It remains questionable whether $\mathrm{Ca}^{2+}$ waves or microdomains directly stimulate $\Delta \Psi_{\mathrm{m}}$ fluctuations.

In favour of a dependency of $\Delta \Psi_{\mathrm{m}}$ fluctuations on $\mathrm{Ca}^{2+}$ are experiments showing that $\mathrm{Ca}^{2+}$ chelators arrest $\Delta \Psi_{\mathrm{m}}$ fluctuations, while limited increase in cytosolic $\mathrm{Ca}^{2+}$ can trigger them (Vergun and Reynolds 2004). $\mathrm{Ca}^{2+}$ stimulates the respiratory chain, which on the other hand increases ROS production. ROS was found to cause propagating wavelike $\Delta \Psi_{\mathrm{m}}$ fluctuations in cardiomyocytes by limited opening of the MPTP, although the direction of fluctuations was towards depolarization (Collins et al. 2002). Similarly in hippocampal neurons 
mitochondria can trigger $\mathrm{Ca}^{2+}$ release from the ER by releasing ROS acting at RyR and $\mathrm{IP}_{3}$ receptors (Gerich et al. 2009). This finding though has not yet been ascertained for astrocytes. The ER in general was found to interact closely with mitochondria. This crosstalk of the two closely colocalized organelles is a topic which is currently in the spotlight of neuroscience (Brookes et al. 2004).

The results obtained in this thesis strongly support the model of cytosolic $\mathrm{Ca}^{2+}$ rises emanating from intracellular ER sources as an activator for intermittently higher respiratory activity of mitochondria becoming evident as $\Delta \Psi_{\mathrm{m}}$ fluctuations. The resulting elevation of ROS production may function both as a propagator of $\Delta \Psi_{\mathrm{m}}$ in the cytosol and as a feedback mechanism to the ER concerning $\mathrm{Ca}^{2+}$ release (Fig. 48).

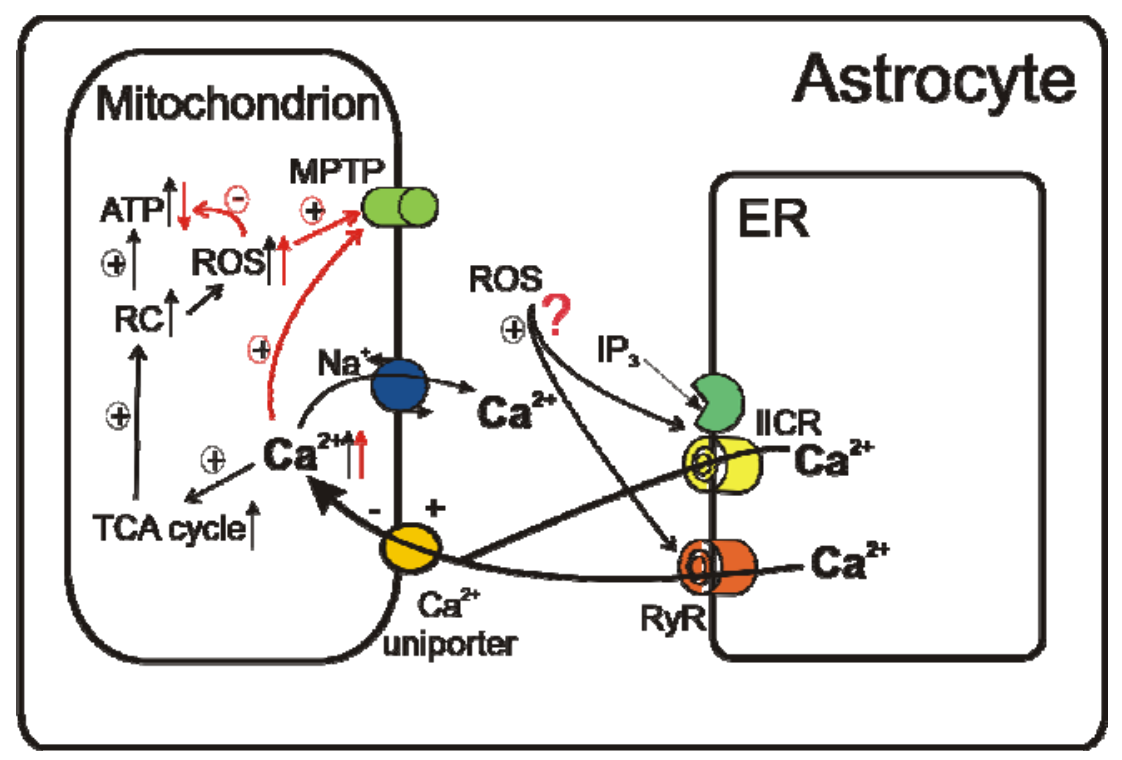

Figure 48 The scheme was remodelled from Fig. 4. It summarizes the close interaction of mitochondria and the ER. Fluctuations in ER Ca ${ }^{2+}$ release from ryanodine receptor (RyR) and $\mathrm{IP}_{3}$ receptor (IICR) dependent pathways lead to variable mitochondrial $\mathrm{Ca}^{2+}$ uptake via a uniporter. Mild elevations of mitochondrial $\mathrm{Ca}^{2+}$ will stimulate the TCA cycle and by delivering more substrate indirectly activate the respiratory chain (RC). This has a direct effect on the proton concentration in the intermembrane space and thus $\Delta \Psi_{\mathrm{m}}$ and may explain the origin of $\Delta \Psi_{\mathrm{m}}$ fluctuations. An activated RC results in higher ROS concentration. Possibly mitochondrially released ROS can stimulate further $E R \mathrm{Ca}^{2+}$ release as found in neurons - a possible explanation for $\mathrm{Ca}^{2+}$ transients (red question mark). However, under pathological conditions $\mathrm{Ca}^{2+}$ and ROS may accumulate in mitochondria in concentrations leading to a decline in ATP production, inhibition of the RC and pro-apoptotic assembly and opening of the mitochondrial transmembrane pore (MPTP).

The underlying physiological reason for this communication yet remains obscure. In particular, it has still not been elucidated whether $\Delta \Psi_{\mathrm{m}}$ fluctuations 
are a manifestation of regular mitochondrial activity at rest or represent a state of extraordinary activation. A possible concept may be an ER induced activation of mitochondria prior to situations of higher ATP demand, e.g. during pronounced neuronal activity. But why can we then note that some mitochondria keep a constant $\Delta \Psi_{m}$ while adjacent mitochondria do not?

Emanating from these questions astrocytes were exposed to stimuli of excitotoxicity and metabolic challenge, which excel the usual stress on astrocytes and their mitochondria during neuronal activity, in order to provoke more pronounced $\Delta \Psi \mathrm{m}$ fluctuations as clear reactions.

\subsection{Heterogeneous reactions of mitochondria to metabolic challenge}

A concept of $\Delta \Psi_{\mathrm{m}}$ fluctuations, which are likely to be maintained by $\mathrm{ER} \mathrm{Ca}^{2+}$ release and are not affected by constant, normal extracellular $\mathrm{Ca}^{2+}$ levels around the astrocyte, can be derived from my results.

The idea was then to simulate an excitotoxic event by exposure to very high glutamate levels. The a priori assumption was that the neurotransmitter might provoke intensified $\Delta \Psi_{\mathrm{m}}$ fluctuations in perhaps even more mitochondria due to a stronger $\mathrm{Ca}^{2+}$ stimulus from the ER via IICR through mGluR or CICR via RyR. The presumptive physiological background could be an activation of mitochondria due to higher ATP demand to buffer the excitotoxic challenge.

Indeed, glutamate in $50 \%$ of experiments would initially not lead to depolarization and a termination of $\Delta \Psi_{\mathrm{m}}$ fluctuations, but sometimes even a slight increase of the number of fluctuating mitochondria was observed. Yet repetitive exposure to glutamate abolished $\Delta \Psi_{\mathrm{m}}$ fluctuations and depolarized mitochondria in most recordings.

A possible explanation is that a limited exposure to glutamate, e. g. by 
moderate concentration of the neurotransmitter, and thus moderately increased cytosolic $\mathrm{Ca}^{2+}$ and $\mathrm{Na}^{+}$levels via AMPA and NMDA receptor activation, will lead to higher activity of $\Delta \Psi_{\mathrm{m}}$ fluctuations in tribute of a higher ATP demand to cope with the buffering of both ions and neurotransmitter. At some point, though, this mechanism turns dysfunctional. A possible reason can be that high levels of ROS generation due to greater transient activation of mitochondria may eventually hamper mitochondrial function and ATP production (Echtay et al. 2002) leading to decreased ATP production rates and impaired function of the astrocyte. At the same time it was shown that the opening probability of the MPTP is increased by high intramitochondrial $\mathrm{Ca}^{2+}$ concentration (Bambrick et al. 2004). Mitochondria are important organelles for $\mathrm{Ca}^{2+}$ buffering in astrocytes. Under pathophysiological circumstances as in extraordinary glutamate release and particularly while ATP production declines this buffering mechanism may then trigger apoptosis.

A confirmation of the depolarizing effect of excessive cytosolic $\mathrm{Ca}^{2+}$ on mitochondria was seen when selectively $\mathrm{Ca}^{2+}$ entered the astrocyte in experiments with either high or extraordinarily low $\mathrm{K}^{+}$concentrations. It then made no difference whether the astrocyte remained polarized - as in $0.2 \mathrm{mM} \mathrm{K}^{+}$ experiments - or showed massive depolarization as expected when applying 50 $\mathrm{mM} \mathrm{K}{ }^{+}$. Both the intermittent activation of some mitochondria, i.e. the $\Delta \Psi_{\mathrm{m}}$ fluctuations, and ratiometrically visualized $\Delta \Psi_{\mathrm{m}}$ were in decline supporting the idea that small elevations of cytosolic $\mathrm{Ca}^{2+}$ may indeed stimulate $\Delta \Psi_{\mathrm{m}}$ fluctuations, but that the excessive buffering of $\mathrm{Ca}^{2+}$ ions will cause the opposite. In hepatocytes it was found, that even under physiological conditions mitochondria can buffer $\mathrm{Ca}^{2+}$ ions - a fact that had long been questioned (Sparagna et al. 1995).

Results of these experiments are favourable of the idea that $\Delta \Psi_{\mathrm{m}}$ fluctuations are part of a physiologic crosstalk of mitochondria and the ER and possibly represent a strategy to satisfy temporary or maybe even local swings in ATP demand, e.g. during episodes of greater demand for astrocytic buffering. To 
give more proof of the declaration that $\Delta \Psi_{\mathrm{m}}$ fluctuations are not a precursor of apoptosis but rather physiological, the respiratory chain was pharmacologically impaired.

All experiments applying $\mathrm{CN}^{-}$, azide or FCCP uniformly unveiled that $\Delta \Psi_{\mathrm{m}}$ fluctuations directly depend on an unabated oxidative phosphorylation. Otherwise the on-off transition of $\Delta \Psi_{\mathrm{m}}$ fluctuations is no longer functional. Similarly all pharmacological inhibitors/uncouplers led to mitochondrial depolarization. Their faster effect in relation to excessive cytosolic $\mathrm{Ca}^{2+}$ accumulation can be explained by a more direct mode of action on the respiratory chain. A recovery from the three drugs applied was more variable. FCCP showed the fastest reaction, but was also the drug with the most reversible effect. For reasons of difficult handling of oligomycin, i.e. tendency to precipitation, I had not applied the drug to prevent ATP depletion and determined all effects of FCCP as derived from mitochondrial depolarization. The primary effect of FCCP is a complete mitochondrial depolarization by shortcircuiting the electrochemical gradient, whereas the complex IV inhibitors azide and $\mathrm{CN}^{-}$first cause a blockade of electron transport and only secondarily a mitochondrial depolarization. Therefore one may argue that loss of $\Delta \Psi_{\mathrm{m}}$ is an important factor in the silencing of $\Delta \Psi_{\mathrm{m}}$ fluctuations and may interrupt the mitochondria/ER crosstalk. Yet ATP depletion will probably eventually cause the same effect of decreased mitochondrial functionality.

Other than previously found I cannot agree that mitochondria in astrocytes are not very vulnerable to $\mathrm{CN}^{-}$(Prabhakaran et al. 2002): The effect of $\mathrm{CN}^{-}$was indeed rather persisting. Yet this metabolic compromise of mitochondria revealed again that some mitochondria are more resistant to ATP depletion than others. They remained polarized despite of a general trend towards depolarization. Similarly some mitochondria would regain $\Delta \Psi_{\mathrm{m}}$ fluctuations after washout of $\mathrm{CN}^{-}$. 


\subsection{Concluding remarks}

These findings together with those of experiments illuminating the distribution of $\Delta \Psi_{\mathrm{m}}$ and the effect of excitotoxicity demonstrate the complexity of the regulation of $\Delta \Psi_{\mathrm{m}}$ in astrocytes. Mechanisms that control the respiratory activity of mitochondria in astrocytes - possibly even self-regulation or intermitochondrial communication - are only poorly understood. In the concentrations used all drugs poisoning the respiratory chain abolished $\Delta \Psi_{\mathrm{m}}$ fluctuations. Yet their influence on kinetic aspects of $\Delta \Psi_{\mathrm{m}}$ fluctuations, e.g. changes in their time course or degree of synchronization, was not observed. The degree of heterogeneity of mitochondria, whether it is a transient heterogeneity or even genetically determined remains obscure. At least functionally mitochondria are probably separated into heterogeneous subsets within the same astrocyte. After all, the results presented in this thesis stress the close interaction of mitochondria and the ER with $\mathrm{Ca}^{2+}$ acting as an inter-organelle messenger. $\Delta \Psi_{\mathrm{m}}$ fluctuations and $\mathrm{Ca}^{2+}$ transients are possible manifestations of it. The role of ROS buffering and release concerning $\Delta \Psi_{\mathrm{m}}$ fluctuations and $\mathrm{ER} \mathrm{Ca}{ }^{2+}$ release in astrocytes will be a question worth elucidating in the near future. 


\section{Summary}

This thesis aimed to reveal deeper insight into the heterogeneity of mitochondria in cultured astrocytes of the neonatal rat hippocampus.

During the past two decades both the role of astrocytes as well as of mitochondria in brain physiology and pathology was object to great reevaluation. Astrocytes were found to be important modulators of neural communication and metabolism and vital for cell survival of neurones. Similarly, the pathogenesis of many neurodegenerative diseases such as Parkinson's disease or various types of dementia was associated with impaired function of the respiratory chain of mitochondria. As a consequence research focused on the impact of mitochondria on cellular energy metabolism, $\mathrm{Ca}^{2+}$ homeostasis and apoptosis in various cell types. On the subcellular level mitochondria proved not to be uniformly reacting organelles but rather heterogeneous in multiple ways.

Here high-resolution imaging applying wide-field microscopy as well as twophoton emission laser scanning microscopy was combined with the advantages of the ratiometric fluorophore $\mathrm{JC}-1$ to elucidate quantitatively and qualitatively the heterogeneity of astrocytic mitochondria as regard to cellular localization, shape, transmembrane potential $\left(\Delta \Psi_{\mathrm{m}}\right)$ and $\mathrm{Ca}^{2+}$ homeostasis under physiologic conditions as well as metabolic compromise as a novel approach for this cell type.

Mitochondria were found to be strategically localized mainly close to the perinuclear endoplasmic reticulum (ER). The application of the image splitter device Dual-View ${ }^{\circledR}$ revealed yet a higher polarization in peripheral mitochondria. Mitochondria would also differ in their reaction towards metabolic compromise by the application of cyanide or FCCP and further pharmacological agents. Frequent fluctuations in $\Delta \Psi_{\mathrm{m}}$ proved to be an innate characteristic of most 
mitochondria and could be associated with unabated mitochondrial function and cytosolic $\mathrm{Ca}^{2+}$ homeostasis in astrocytes after experiments with dantrolene and 2-APB as blockers of ryanodine and $\mathrm{IP}_{3}$ receptors on the ER respectively since the corresponding experiments applying the $\mathrm{Ca}^{2+}$ indicator Fluo-3 revealed a suppression of $\mathrm{Ca}^{2+}$ waves in astrocytes. Furthermore rhythmic fluctuations of $\Delta \Psi_{\mathrm{m}}$ were for the first time analyzed concerning their spatiotemporal pattern in real-time making use of the two discrimination of $\Delta \Psi_{\mathrm{m}}$ by the potentiometric probe $\mathrm{JC}-1$, which emits at two different wavelengths, revealing synchronized fluctuations of varying kinetics and spatial extent. A correlation analysis of $\Delta \Psi_{\mathrm{m}}$ and aspects of distribution and shape of mitochondria completed this thesis.

In conclusion, these findings demonstrate that mitochondria are crucially involved in and reactive to changes in astrocytic metabolism and at the same time prove once again to be very autonomous and individually reacting organelles even on the subcellular level. 


\section{Literature}

Allen NJ and Barres BA (2005): Signaling between glia and neurons: focus on synaptic plasticity. Curr Opin Neurobiol 15 , 542-548

Amaral D and Lavenex P: Comparative Neuroanatomy of the Rat, Monkey, and Human Hippocampal Formation; in: The Hippocampus Book, Ch. 3 - Hippocampal Neuroanatomy; Ed. by Andersen P, Morris R, Amaral D, Bliss T and O'Keefe J; Oxford University Press, New York 2007, 95 - 107

Andrews ZB, Diano S and Horvath TL (2005): Mitochondrial uncoupling proteins in the CNS: in support of function and survival. Nat Rev Neurosci $\underline{6}$, 829-840

Babcock DF, Herrington J, Goodwin PC, Park YB and Hille B (1997):

Mitochondrial participation in the intracellular $\mathrm{Ca} 2+$ network. J Cell Biol $\underline{136}$, 833-844

Baloyannis SJ (2006): Mitochondrial alterations in Alzheimer's disease. J Alzheimers Dis $\underline{9}, 119-126$

Bambrick L, Kristian T and Fiskum G (2004): Astrocyte mitochondrial mechanisms of ischemic brain injury and neuroprotection. Neurochem Res $\underline{29}$, 601608

Baron JC (2001): Perfusion thresholds in human cerebral ischemia: historical perspective and therapeutic implications. Cerebrovasc Dis 11 Suppl 1, 2-8

Basarsky TA, Duffy SN, Andrew RD and MacVicar BA (1998): Imaging spreading depression and associated intracellular calcium waves in brain slices. $J$ Neurosci 18, 7189-7199

Beal MF (2005): Mitochondria take center stage in aging and neurodegeneration. Ann Neurol $\underline{58}, 495-505$

Bechterew WM (1900): Demonstration eines Gehirns mit Zerstörung der vorderen und inneren Theile der Hirnrinde beider Schläfenlappen. Neurol Zentralbl 19, 990-991

Belousov VV, Bambrick LL, Starkov AA, Zorov DB, Skulachev VP and Fiskum $G$ (2001): Oscillations in mitochondrial membrane potential in rat astrocytes in vitro. Soc Neurosci Abstr 27, 205.218

Benzi G and Moretti A (1995): Are reactive oxygen species involved in Alzheimer's disease? Neurobiol Aging $\underline{16}, 661-674$ 
Bereiter-Hahn J and Voth M (1994): Dynamics of mitochondria in living cells: shape changes, dislocations, fusion, and fission of mitochondria. Microsc Res Tech $\underline{27}, 198-219$

Bereiter-Hahn J, Seipel KH, Voth M and Ploem JS (1983): Fluorimetry of mitochondria in cells vitally stained with DASPMI or rhodamine $6 \mathrm{GO}$. Cell Biochem Funct 1, 147-155

Berridge MJ (1993): Inositol trisphosphate and calcium signalling. Nature $\underline{361}$, 315-325

Berridge MJ and Galione A (1988): Cytosolic calcium oscillators. FASEB J $\underline{2}$, 3074-3082

Bertoni-Freddari C, Fattoretti P, Paoloni R, Caselli U, Giorgetti B and Solazzi M (2003): Inverse correlation between mitochondrial size and metabolic competence: a quantitative cytochemical study of cytochrome oxidase activity. Naturwissenschaften $\underline{90}, 68-71$

Bignami A (1991): Glial cells in the central nervous system. Discuss Neurosci $\underline{8}$, $1-45$

Bindokas VP, Lee CC, Colmers WF and Miller RJ (1998): Changes in mitochondrial function resulting from synaptic activity in the rat hippocampal slice. $\mathrm{J}$ Neurosci $\underline{18}, 4570-4587$

Bonita R and Reddy S: Cardiovascular diseases: the need to act; in: World Health Report 2003. Shaping the future, Ch. 6 - Neglected global epidemics: three growing threats; Ed. by Evans T and Beaglehole R; World Health Organization, Geneva 2003, 85

Bos MJ, van Rijn MJ, Witteman JC, Hofman A, Koudstaal PJ and Breteler MM (2007): Incidence and prognosis of transient neurological attacks. JAMA $\underline{298}$, 2877-2885

Brookes PS, Yoon Y, Robotham JL, Anders MW and Sheu SS (2004): Calcium, ATP, and ROS: a mitochondrial love-hate triangle. Am J Physiol Cell Physiol 287, C817-833

Butt AM and Kalsi A (2006): Inwardly rectifying potassium channels (Kir) in central nervous system glia: a special role for Kir4.1 in glial functions. J Cell Mol Med 10, 33-44

Chan DC (2006): Mitochondria: dynamic organelles in disease, aging, and development. Cell 125, 1241-1252 
Charles AC, Merrill JE, Dirksen ER and Sanderson MJ (1991): Intercellular signaling in glial cells: calcium waves and oscillations in response to mechanical stimulation and glutamate. Neuron $\underline{6}, 983-992$

Charles AC, Dirksen ER, Merrill JE and Sanderson MJ (1993): Mechanisms of intercellular calcium signaling in glial cells studied with dantrolene and thapsigargin. Glia $\underline{7}, 134-145$

Chen H, Chomyn A and Chan DC (2005): Disruption of fusion results in mitochondrial heterogeneity and dysfunction. J Biol Chem 280, 26185-26192

Chen Y and Swanson RA (2003): Astrocytes and brain injury. J Cereb Blood Flow Metab 23, 137-149

Cherubini E, Ben-Ari Y and Krnjevic K (1989): Anoxia produces smaller changes in synaptic transmission, membrane potential, and input resistance in immature rat hippocampus. J Neurophysiol $\underline{62}, 882-895$

Christie-Pope BC, Burns RS and Whetsell WO, Jr. (1989): Ultrastructural alterations induced by 1-methyl-4-phenylpyridinium (MPP+) in canine substantia nigra and rat mesencephalon in vitro. Exp Neurol 104, 235-240

Clapham DE (2007): Calcium signaling. Cell 131, 1047-1058

Collins TJ, Berridge MJ, Lipp P and Bootman MD (2002): Mitochondria are morphologically and functionally heterogeneous within cells. EMBO J $\underline{21}, 1616-$ 1627

Cornell-Bell AH, Finkbeiner SM, Cooper MS and Smith SJ (1990): Glutamate induces calcium waves in cultured astrocytes: long-range glial signaling.

Science 247, 470-473

Cossarizza A, Ceccarelli D and Masini A (1996): Functional heterogeneity of an isolated mitochondrial population revealed by cytofluorometric analysis at the single organelle level. Exp Cell Res 222, 84-94

Dallwig R, Vitten H and Deitmer JW (2000): A novel barium-sensitive calcium influx into rat astrocytes at low external potassium. Cell Calcium $\underline{28}, 247-259$

Dani JW, Chernjavsky A and Smith SJ (1992): Neuronal activity triggers calcium waves in hippocampal astrocyte networks. Neuron $\underline{8}, 429-440$

Davolio C and Greenamyre JT (1995): Selective vulnerability of the CA1 region of hippocampus to the indirect excitotoxic effects of malonic acid. Neurosci Lett 192, 29-32

Denk W, Strickler JH and Webb WW (1990): Two-photon laser scanning fluorescence microscopy. Science $\underline{248}, 73-76$ 
Di Lisa F, Blank PS, Colonna R, Gambassi G, Silverman HS, Stern MD and Hansford RG (1995): Mitochondrial membrane potential in single living adult rat cardiac myocytes exposed to anoxia or metabolic inhibition. J Physiol $\underline{486}$ (Pt 1), $1-13$

Diaz G, Setzu MD, Zucca A, Isola R, Diana A, Murru R, Sogos V and Gremo F (1999): Subcellular heterogeneity of mitochondrial membrane potential: relationship with organelle distribution and intercellular contacts in normal, hypoxic and apoptotic cells. J Cell Sci 112 (Pt 7), 1077-1084

Diaz G, Falchi AM, Gremo F, Isola R and Diana A (2000): Homogeneous longitudinal profiles and synchronous fluctuations of mitochondrial transmembrane potential. FEBS Lett $\underline{475}, 218-224$

Donnelly DF, Jiang C and Haddad GG (1992): Comparative responses of brain stem and hippocampal neurons to $\mathrm{O} 2$ deprivation: in vitro intracellular studies. Am J Physiol 262, L549-554

Dougherty KD, Dreyfus CF and Black IB (2000): Brain-derived neurotrophic factor in astrocytes, oligodendrocytes, and microglia/macrophages after spinal cord injury. Neurobiol Dis $\underline{7}, 574-585$

Duchen MR (1999): Contributions of mitochondria to animal physiology: from homeostatic sensor to calcium signalling and cell death. J Physiol $\underline{516}$ (Pt 1), 117

Duchen MR, Leyssens A and Crompton M (1998): Transient mitochondrial depolarizations reflect focal sarcoplasmic reticular calcium release in single rat cardiomyocytes. J Cell Biol 142, 975-988

Duchen MR, Surin A and Jacobson J (2003): Imaging mitochondrial function in intact cells. Methods Enzymol 361, 353-389

Echtay KS, Roussel D, St-Pierre J, Jekabsons MB, Cadenas S, Stuart JA, Harper JA, Roebuck SJ, Morrison A, Pickering S et al. (2002): Superoxide activates mitochondrial uncoupling proteins. Nature $\underline{415}, 96-99$

Ekstrom AD, Kahana MJ, Caplan JB, Fields TA, Isham EA, Newman EL and Fried I (2003): Cellular networks underlying human spatial navigation. Nature $\underline{425}, 184-188$

Emaus RK, Grunwald R and Lemasters JJ (1986): Rhodamine 123 as a probe of transmembrane potential in isolated rat-liver mitochondria: spectral and metabolic properties. Biochim Biophys Acta $\underline{850}$, 436-448

Erdemli G, Xu YZ and Krnjevic K (1998): Potassium conductance causing hyperpolarization of CA1 hippocampal neurons during hypoxia. J Neurophysiol $\underline{80}, 2378-2390$ 
Erecinska M and Silver IA (1989): ATP and brain function. J Cereb Blood Flow Metab $\underline{9}, 2-19$

Fellin T and Carmignoto G (2004): Neurone-to-astrocyte signalling in the brain represents a distinct multifunctional unit. J Physiol $\underline{559}$, 3-15

Finkbeiner S (1992): Calcium waves in astrocytes-filling in the gaps. Neuron $\underline{8}$, 1101-1108

Finkbeiner S (1993): Glial calcium. Glia $\underline{9}, 83-104$

Fischer $\mathrm{G}$ and Kettenmann $\mathrm{H}$ (1985): Cultured astrocytes form a syncytium after maturation. Exp Cell Res 159, 273-279

Foster KA, Galeffi F, Gerich FJ, Turner DA and Müller M (2006): Optical and pharmacological tools to investigate the role of mitochondria during oxidative stress and neurodegeneration. Prog Neurobiol $\underline{79}$, 136-171

Frosch M, Anthony D and Girolami UD: Cerebrovascular Diseases; in: Robbins \& Cotran Pathologic Basis of Disease, Ch. 28: The Central Nervous System; Ed. by Kumar V, Fausto N and Abbas A; W.B. Saunders Company, Philadelphia 2004, $1361-1363$

Gerich FJ, Funke F, Hildebrandt B, Fasshauer M and Muller M (2009):

$\mathrm{H}(2) \mathrm{O}(2)$-mediated modulation of cytosolic signaling and organelle function in rat hippocampus. Pflugers Arch $\underline{458}, 937-952$

Gibson GE, Pulsinelli W, Blass JP and Duffy TE (1981): Brain dysfunction in mild to moderate hypoxia. Am J Med $\underline{70}, 1247-1254$

Göppert-Mayer M (1931): Über Elementarakte mit zwei Quantensprüngen. Ann Phys $\underline{401}$, 273-294

Gordon GR, Choi HB, Rungta RL, Ellis-Davies GC and MacVicar BA (2008): Brain metabolism dictates the polarity of astrocyte control over arterioles. Nature $\underline{456}, 745-749$

Gunter TE and Pfeiffer DR (1990): Mechanisms by which mitochondria transport calcium. Am J Physiol 258, C755-786

Guthrie PB, Knappenberger J, Segal M, Bennett MV, Charles AC and Kater SB (1999): ATP released from astrocytes mediates glial calcium waves. J Neurosci $\underline{19}, 520-528$

Hansson E and Ronnback L (2003): Glial neuronal signaling in the central nervous system. FASEB J 17, 341-348 
Hartel K, Singaravelu K, Kaiser M, Neusch C, Hulsmann S and Deitmer JW (2007): Calcium influx mediated by the inwardly rectifying $\mathrm{K}+$ channel Kir4.1 $(\mathrm{KCNJ} 10)$ at low external $\mathrm{K}+$ concentration. Cell Calcium $42,271-280$

Haydon PG (2001): GLIA: listening and talking to the synapse. Nat Rev Neurosci 2, 185-193

Hepp S, Gerich FJ and Müller M (2005): Mitochondrial depolarization prior to oxygen withdrawal facilitates the occurence of hypoxic spreading depression. Society for Neuroscience, Abstract Viewer/Itinerary Planner 550.551

Hertz L, Dringen R, Schousboe A and Robinson SR (1999): Astrocytes: glutamate producers for neurons. J Neurosci Res $\underline{57}$, 417-428

Hirase H, Qian L, Bartho P and Buzsaki G (2004): Calcium dynamics of cortical astrocytic networks in vivo. PLoS Biol 2, E96

Hollenbeck PJ and Saxton WM (2005): The axonal transport of mitochondria. J Cell Sci 118, 5411-5419

Hossmann KA (2008): Cerebral Ischemia: Models, methods and outcomes. Neuropharmacology $\underline{55}, 257-270$

Ichas F, Jouaville LS and Mazat JP (1997): Mitochondria are excitable organelles capable of generating and conveying electrical and calcium signals. Cell $\underline{89}$, 1145-1153

Jensen JR and Rehder V (1991): FCCP releases Ca2+ from a non-mitochondrial store in an identified Helisoma neuron. Brain Res $\underline{551}$, 311-314

Jonas EA, Buchanan J and Kaczmarek LK (1999): Prolonged activation of mitochondrial conductances during synaptic transmission. Science $\underline{286}$, 13471350

Jouaville LS, Ichas F, Holmuhamedov EL, Camacho P and Lechleiter JD (1995): Synchronization of calcium waves by mitochondrial substrates in Xenopus laevis oocytes. Nature $\underline{377}, 438-441$

Kahlert S, Schild L and Reiser G (2001): Mitochondrial polarization in rat hippocampal astrocytes is resistant to cytosolic $\mathrm{Ca}^{2+}$ loads. J Neurosci Res $\underline{66}, 1019-$ 1027

Karbowski M, Kurono C, Nishizawa Y, Horie Y, Soji T and Wakabayashi T (1997): Induction of megamitochondria by some chemicals inducing oxidative stress in primary cultured rat hepatocytes. Biochim Biophys Acta 1349, 242-250

Kirichok Y, Krapivinsky G and Clapham DE (2004): The mitochondrial calcium uniporter is a highly selective ion channel. Nature $\underline{427}, 360-364$ 
Kostyuk P and Verkhratsky A (1994): Calcium stores in neurons and glia. Neuroscience $\underline{63}, 381-404$

Kroemer G and Reed JC (2000): Mitochondrial control of cell death. Nat Med $\underline{6}$, 513-519

Kuznetsov AV and Margreiter R (2009): Heterogeneity of Mitochondria and Mitochondrial Function within Cells as Another Level of Mitochondrial Complexity. Int J Mol Sci $\underline{10}$, 1911-1929

Lai JC, Leung TK and Lim L (1994): Heterogeneity of monoamine oxidase activities in synaptic and non-synaptic mitochondria derived from three brain regions: some functional implications. Metab Brain Dis $\underline{9}$, 53-66

Langley D and Pearce B (1994): Ryanodine-induced intracellular calcium mobilisation in cultured astrocytes. Glia $\underline{12}, 128-134$

Lebuffe G, Schumacker PT, Shao ZH, Anderson T, Iwase H and Vanden Hoek TL (2003): ROS and NO trigger early preconditioning: relationship to mitochondrial KATP channel. Am J Physiol Heart Circ Physiol 284, H299-308

Lemasters $\mathrm{J}$ and Ramshesh V: III Assays for Mitochondrial Respiratory Activity in Living Cells - Ch. 14: Imaging of Mitochondrial Polarization and Depolarization with Cationic Fluorophores; in: Methods in Cell Biology, 80 Mitochondria; Ed. by Pon LA and Schon EA; Elsevier, Academic Press, Amsterdam 2007, 289

Leonard J and Schapira A (2000): Mitochondrial respiratory chain disorders II: neurodegenerative disorders and nuclear gene defects. The Lancet $\underline{355}, 389$ 394

Lin MT and Beal MF (2006): Mitochondrial dysfunction and oxidative stress in neurodegenerative diseases. Nature $\underline{443}, 787-795$

Loew LM, Tuft RA, Carrington W and Fay FS (1993): Imaging in five dimensions: time-dependent membrane potentials in individual mitochondria. Biophys $\mathrm{J} \underline{65}$, 2396-2407

Luo Y, Bond JD and Ingram VM (1997): Compromised mitochondrial function leads to increased cytosolic calcium and to activation of MAP kinases. Proc Natl Acad Sci U S A 94, 9705-9710

Magistretti PJ (2006): Neuron-glia metabolic coupling and plasticity. J Exp Biol $\underline{209}, 2304-2311$

Margineantu DH, Brown RM, Brown GK, Marcus AH and Capaldi RA (2002): Heterogeneous distribution of pyruvate dehydrogenase in the matrix of mitochondria. Mitochondrion 1, 327-338 
Martin W and Kowallik KV (1999): Annotated English translation of Mereschkowsky's 1905 paper 'Über Natur und Ursprung der Chromatophoren im Pflanzenreiche'. Eur J Phycol 34, 287-295

Martinelli C, Sartori P, Ledda M and Pannese E (2003): Age-related quantitative changes in mitochondria of satellite cell sheaths enveloping spinal ganglion neurons in the rabbit. Brain Res Bull $\underline{61}, 147-151$

Marx P: 40 Zerebrale und spinale Ischämie; in: Neurologie - Diagnostik und Therapie in Klinik und Praxis, Sec. III ZNS-Erkrankungen; Ed. by Wallesch C-W; Elsevier GmbH, München 2005, 471

Mata M and Fink DJ (1989): Ca++-ATPase in the central nervous system: an EM cytochemical study. J Histochem Cytochem $\underline{37}$, 971-980

Mathur A, Hong Y, Kemp BK, Barrientos AA and Erusalimsky JD (2000): Evaluation of fluorescent dyes for the detection of mitochondrial membrane potential changes in cultured cardiomyocytes. Cardiovasc Res $\underline{46}, 126-138$

Matute C, Domercq M and Sanchez-Gomez MV (2006): Glutamate-mediated glial injury: mechanisms and clinical importance. Glia $\underline{53}, 212-224$

Milani D, Facci L, Guidolin D, Leon A and Skaper SD (1989): Activation of polyphosphoinositide metabolism as a signal-transducing system coupled to excitatory amino acid receptors in astroglial cells. Glia $\underline{2}, 161-169$

Mironov SL (2007): ADP regulates movements of mitochondria in neurons. Biophys J 92, 2944-2952

Mitchell P (1966): Chemiosmotic coupling in oxidative and photosynthetic phosphorylation. Biol Rev Camb Philos Soc 41, 445-502

Mouli PK, Twig G and Shirihai OS (2009): Frequency and selectivity of mitochondrial fusion are key to its quality maintenance function. Biophys $\mathrm{J} \underline{\mathbf{9 6}}$, 3509-3518

Müller M, Schmidt J, Mironov SL and Richter DW (2003): Construction and performance of a custom-built two-photon laser scanning system. J Phys D: Appl Phys $\underline{36}$, 1747-1757

Müller M, Mironov SL, Ivannikov MV, Schmidt J and Richter DW (2005): Mitochondrial organization and motility probed by two-photon microscopy in cultured mouse brainstem neurons. Exp Cell Res $\underline{303}, 114-127$

Nadarajah B, Thomaidou D, Evans WH and Parnavelas JG (1996): Gap junctions in the adult cerebral cortex: regional differences in their distribution and cellular expression of connexins. J Comp Neurol 376, 326-342 
Nedergaard M (1994): Direct signaling from astrocytes to neurons in cultures of mammalian brain cells. Science $\underline{263}, 1768-1771$

Nicholls DG (1985): A role for the mitochondrion in the protection of cells against calcium overload? Prog Brain Res $\underline{63}$, 97-106

Nicholls DG, Budd SL, Castilho RF and Ward MW (1999): Glutamate excitotoxicity and neuronal energy metabolism. Ann N Y Acad Sci 893, 1-12

O'Reilly CM, Fogarty KE, Drummond RM, Tuft RA and Walsh JV, Jr. (2003): Quantitative analysis of spontaneous mitochondrial depolarizations. Biophys $\mathrm{J}$ $\underline{85}, 3350-3357$

Park MK, Ashby MC, Erdemli G, Petersen OH and Tepikin AV (2001): Perinuclear, perigranular and sub-plasmalemmal mitochondria have distinct functions in the regulation of cellular calcium transport. EMBO J 20, 1863-1874

Parpura V, Scemes E and Spray DC (2004): Mechanisms of glutamate release from astrocytes: gap junction "hemichannels", purinergic receptors and exocytotic release. Neurochem Int $\underline{45}, 259-264$

Perez-Pinzon MA, Mumford PL, Rosenthal M and Sick TJ (1997): Antioxidants, mitochondrial hyperoxidation and electrical recovery after anoxia in hippocampal slices. Brain Res $\underline{754}$, 163-170

Peuchen S, Clark JB and Duchen MR (1996): Mechanisms of intracellular calcium regulation in adult astrocytes. Neuroscience $\underline{71}, 871-883$

Pivovarova NB, Pozzo-Miller LD, Hongpaisan J and Andrews SB (2002): Correlated calcium uptake and release by mitochondria and endoplasmic reticulum of CA3 hippocampal dendrites after afferent synaptic stimulation. $J$ Neurosci $\underline{22}, 10653-10661$

Pizzo P and Pozzan T (2007): Mitochondria-endoplasmic reticulum choreography: structure and signaling dynamics. Trends Cell Biol $\underline{17}, 511-517$

Porter JT and McCarthy KD (1995): GFAP-positive hippocampal astrocytes in situ respond to glutamatergic neuroligands with increases in [Ca2+]i. Glia $\underline{13}$, 101-112

Porter JT and McCarthy KD (1997): Astrocytic neurotransmitter receptors in situ and in vivo. Prog Neurobiol $\underline{51}$, 439-455

Pozzo-Miller LD, Pivovarova NB, Leapman RD, Buchanan RA, Reese TS and Andrews SB (1997): Activity-dependent calcium sequestration in dendrites of hippocampal neurons in brain slices. J Neurosci 17, 8729-8738 
Prabhakaran K, Li L, Borowitz JL and Isom GE (2002): Cyanide induces differrent modes of death in cortical and mesencephalon cells. J Pharmacol Exp Ther $\underline{303}, 510-519$

Ransom B, Behar T and Nedergaard M (2003): New roles for astrocytes (stars at last). Trends Neurosci $\underline{26}, 520-522$

Rego AC and Oliveira CR (2003): Mitochondrial dysfunction and reactive oxygen species in excitotoxicity and apoptosis: implications for the pathogenesis of neurodegenerative diseases. Neurochem Res $\underline{28}$, 1563-1574

Repine JE, Pfenninger OW, Talmage DW, Berger EM and Pettijohn DE (1981): Dimethyl sulfoxide prevents DNA nicking mediated by ionizing radiation or iron/ hydrogen peroxide-generated hydroxyl radical. Proc Natl Acad Sci U S A $\underline{78}$, 1001-1003

RGSP Consortium (2004): Genome sequence of the Brown Norway rat yields insights into mammalian evolution. Nature $\underline{428}, 493-521$

Rizzuto R, Brini M, Murgia M and Pozzan T (1993): Microdomains with high $\mathrm{Ca} 2+$ close to IP3-sensitive channels that are sensed by neighboring mitochondria. Science 262, 744-747

Ross DT and Graham DI (1993): Selective loss and selective sparing of neurons in the thalamic reticular nucleus following human cardiac arrest. $J$ Cereb Blood Flow Metab 13, 558-567

Salvioli S, Ardizzoni A, Franceschi C and Cossarizza A (1997): JC-1, but not DiOC6(3) or rhodamine 123, is a reliable fluorescent probe to assess delta psi changes in intact cells: implications for studies on mitochondrial functionality during apoptosis. FEBS Lett $\underline{411}$, 77-82

Scaduto RC, Jr. and Grotyohann LW (1999): Measurement of mitochondrial membrane potential using fluorescent rhodamine derivatives. Biophys $\mathrm{J} \underline{76}$, 469-477

Scemes E and Giaume C (2006): Astrocyte calcium waves: what they are and what they do. Glia $\underline{54}, 716-725$

Schild L, Huppelsberg J, Kahlert S, Keilhoff G and Reiser G (2003): Brain mitochondria are primed by moderate $\mathrm{Ca} 2+$ rise upon hypoxia/reoxygenation for functional breakdown and morphological disintegration. J Biol Chem $\underline{278}$, 25454-25460

Schmidt-Kastner R and Freund TF (1991): Selective vulnerability of the hippocampus in brain ischemia. Neuroscience $\underline{40}, 599-636$ 
Scoville WB and Milner B (1957): Loss of recent memory after bilateral hippocampal lesions. J Neurol Neurosurg Psychiatry 20, 11-21

Sheppard C (1990): Image formation in two-photon fluorescence microscopy. Optik 6ㅜ, 104-106

Siemens A, Walter R, Liaw LH and Berns MW (1982): Laser-stimulated fluorescence of submicrometer regions within single mitochondria of rhodamine-treated myocardial cells in culture. Proc Natl Acad Sci U S A $\underline{79}, 466-470$

Sinor AD, Irvin SM, Cobbs CS, Chen J, Graham SH and Greenberg DA (1998): Hypoxic induction of vascular endothelial growth factor (VEGF) protein in astroglial cultures. Brain Res $\underline{812}$, 289-291

Skulachev VP (2001): Mitochondrial filaments and clusters as intracellular power-transmitting cables. Trends Biochem Sci $\underline{26}$, 23-29

Smiley ST, Reers M, Mottola-Hartshorn C, Lin M, Chen A, Smith TW, Steele GD, Jr. and Chen LB (1991): Intracellular heterogeneity in mitochondrial membrane potentials revealed by a J-aggregate-forming lipophilic cation JC-1. Proc Natl Acad Sci U S A $\underline{88}, 3671-3675$

Smith ML, Auer RN and Siesjo BK (1984): The density and distribution of ischemic brain injury in the rat following 2-10 min of forebrain ischemia. Acta Neuropathol $\underline{64}, 319-332$

Solenski NJ, diPierro CG, Trimmer PA, Kwan AL and Helm GA (2002): Ultrastructural changes of neuronal mitochondria after transient and permanent cerebral ischemia. Stroke $\underline{33}, 816-824$

Sparagna GC, Gunter KK, Sheu SS and Gunter TE (1995): Mitochondrial calcium uptake from physiological-type pulses of calcium. A description of the rapid uptake mode. J Biol Chem $\underline{270}$, 27510-27515

Spielmeyer W (1927): Die Pathogenese des epileptischen Krampfes. Histopathologischer Teil. Z Ges Neurol Psychiatrie 109, 501-520

Szabados T, Dul C, Majtenyi K, Hargitai J, Penzes Z and Urbanics R (2004): A chronic Alzheimer's model evoked by mitochondrial poison sodium azide for pharmacological investigations. Behav Brain Res 154, 31-40

Takano T, Oberheim N, Cotrina ML and Nedergaard M (2009): Astrocytes and ischemic injury. Stroke $\underline{40}$, S8-12

Trimmer PA, Swerdlow RH, Parks JK, Keeney P, Bennett JP, Jr., Miller SW, Davis RE and Parker WD, Jr. (2000): Abnormal mitochondrial morphology in sporadic Parkinson's and Alzheimer's disease cybrid cell lines. Exp Neurol 162, $37-50$ 
Tsacopoulos M and Magistretti PJ (1996): Metabolic coupling between glia and neurons. J Neurosci 16 , $877-885$

Ullian EM, Sapperstein SK, Christopherson KS and Barres BA (2001): Control of synapse number by glia. Science $\underline{291}, 657-661$

Vega C, R. Sachleben L J, Gozal D and Gozal E (2006): Differential metabolic adaptation to acute and long-term hypoxia in rat primary cortical astrocytes. $\mathrm{J}$ Neurochem 97, 872-883

Vergun O and Reynolds IJ (2004): Fluctuations in mitochondrial membrane potential in single isolated brain mitochondria: modulation by adenine nucleotides and $\mathrm{Ca}^{2+}$. Biophys $\mathrm{J}$ 87, 3585-3593

Vergun O, Votyakova TV and Reynolds IJ (2003): Spontaneous changes in mitochondrial membrane potential in single isolated brain mitochondria. Biophys $\mathrm{J} \underline{85}, 3358-3366$

Verkhratsky A and Kettenmann H (1996): Calcium signalling in glial cells. Trends Neurosci $\underline{19}$, 346-352

Verkhratsky A and Kirchhoff F (2007): NMDA Receptors in glia. Neuroscientist $\underline{13}, 28-37$

Verkhratsky A, Orkand RK and Kettenmann H (1998): Glial calcium: homeostasis and signaling function. Physiol Rev $\underline{78}, 99-141$

Votyakova TV and Reynolds IJ (2001): DeltaPsi(m)-Dependent and - independent production of reactive oxygen species by rat brain mitochondria. $J$ Neurochem $\underline{79}, 266-277$

Waagepetersen HS, Hansen GH, Fenger K, Lindsay JG, Gibson G and Schousboe A (2006): Cellular mitochondrial heterogeneity in cultured astrocytes as demonstrated by immunogold labeling of alpha-ketoglutarate dehydrogenase. Glia $\underline{53}, 225-231$

Walz W (1989): Role of glial cells in the regulation of the brain ion microenvironment. Prog Neurobiol 33, 309-333

Walz W and Wilson DC (1986): Calcium entry into cultured mouse astrocytes. Neurosci Lett $\underline{67}, 301-306$

Werth JL and Thayer SA (1994): Mitochondria buffer physiological calcium loads in cultured rat dorsal root ganglion neurons. J Neurosci 14 , 348-356

Wu H, Friedman WJ and Dreyfus CF (2004): Differential regulation of neurotrophin expression in basal forebrain astrocytes by neuronal signals. J Neurosci Res $\underline{76}, 76-85$ 
Yi M, Weaver D and Hajnoczky G (2004): Control of mitochondrial motility and distribution by the calcium signal: a homeostatic circuit. J Cell Biol 167, 661-672 


\section{Acknowledgements}

I kindly give thanks to Dr. Frank Funke and Belinda Hildebrandt without whose outstanding tuition in imaging techniques and cell culture preparation this thesis could not have been realised.

Many thanks also to Jan Willem Roepert and Luise Erpenbeck for critical reading of this thesis and layout advice.

And finally I would like to thank my Doktorvater Juniorprofessor Dr. rer. nat. Michael Müller for helping me make my first steps in neuroscience. 


\section{Curriculum Vitae}

My name is Vera Catharina Keil. I was born in Münster (Germany) on the $29^{\text {th }}$ of April 1985.

In 2004 I graduated at the Albert-Einstein-Gymnasium (Buchholz i. d. N., Germany; allgemeine Hochschulreife) and enrolled for Medical School in Göttingen (UMG) in Autumn (Wintersemester 2004/05). After a pre-clinical examination (1. ärztliche Prüfung) I entered the clinical part of Göttingen Medical School in Autumn 2006 (Wintersemester 2006/07) and was able to progress into the practical year (PJ; internship) by August 2009.

I spent four months in Internal Medicine at the UMG (Prof. Dr.med. Dr.h.c. G. Ramadori; Prof. Dr.med. G. Hasenfuß) and Endokrinologikum Göttingen (Prof. Dr.med. H. Siggelkow). From December 2009 on I have been an intern at Geneva University Hospitals (HUG), Department of Cardiovascular Surgery (director Prof. A. Kalangos, MD, PhD, FECTS). My last four months of internship will be spent at the Oxford Neuroscience Institute (UK) and Rijksuniversiteit Groningen (NL) - both for Neurosurgery.

Clerkships between 2006 and 2009 entail Anaesthesia (Hôpital Erasme, Brussels/BE), General Practitioning, Rural Community Medicine (Gauri Shankar Hospital, Nepal), Neurology (Neuruppiner Kliniken, D) as well as Geriatric Psychiatry (Fulbrook Centre, Oxford/UK) and Neurosurgery (Göttingen (UMG)).

In December 2006 I commenced preparatory work for this thesis in JProf. Dr. M. Müller's lab group at the Centre of Physiology and Pathophysiology. Experiments began three months later.

In August 2009 I visited Prof. Dr. Dr. P. Tass' group for neuromodulation (INM-7) at the Forschungszentrum Jülich and the Clinic for Stereotaxy and functional Neurosurgery, University of Cologne.

From 2005 on I gathered teaching experience during my continuous assistance in neurophysiology courses (studentische Hilfskraft). 
My studies and clerkships were supported by German National Academic Foundation (Studienstiftung des deutschen Volkes) and the UMG Stipendienprogramm "gö4med".

A publication is currently submitted to an internationally renowned journal. Parts of the data have already been presented at various international Physiological and Neuroscience Meetings:

Müller M, Keil VC and Funke $F$ (2009). Mitochondrial diversity and $\Delta \Psi_{m}$ oscillations as revealed by one- and multiphoton imaging. Proceedings of the $8^{\text {th }}$ Göttingen Meeting of the German Neuroscience Society, T9-1B.

Keil VC, Funke $F$ and Müller M (2008). Subcellular mitochondrial heterogeneity in hippocampal glial cells. Neuroprotection and Neurorepair: Cerebral Ischemia and Stroke. $5^{\text {th }}$ International Symposium, Magdeburg, 55.

Müller M, Keil VC and Funke $F$ (2008). Subcellular mitochondrial heterogeneity in hippocampal glial cells. Acta Physiologica 192 (Supplement 663), PM01-06.

Keil VC and Müller M (2007). Subcellular mitochondrial heterogeneity in hippocampal glial cells. Society for Neuroscience Abstracts, 602.1. 
